\title{
Exploring flavor-dependent long-range forces in long-baseline neutrino oscillation experiments
}

\author{
Sabya Sachi Chatterjee, ${ }^{a}$ Arnab Dasgupta ${ }^{a, b}$ and Sanjib Kumar Agarwalla ${ }^{a}$ \\ ${ }^{a}$ Institute of Physics, \\ Sachivalaya Marg, Sainik School Post, Bhubaneswar 751005, India \\ ${ }^{b}$ Centre for Theoretical Physics, Jamia Millia Islamia-Central University, \\ Jamia Nagar, New Delhi - 110025, India \\ E-mail: sabya@iopb.res.in, arnab.d@iopb.res.in, sanjib@iopb.res.in
}

ABSTRACT: The Standard Model gauge group can be extended with minimal matter content by introducing anomaly free U(1) symmetry, such as $L_{e}-L_{\mu}$ or $L_{e}-L_{\tau}$. If the neutral gauge boson corresponding to this abelian symmetry is ultra-light, then it will give rise to flavor-dependent long-range leptonic force, which can have significant impact on neutrino oscillations. For an instance, the electrons inside the Sun can generate a flavor-dependent long-range potential at the Earth surface, which can suppress the $\nu_{\mu} \rightarrow \nu_{e}$ appearance probability in terrestrial experiments. The sign of this potential is opposite for anti-neutrinos, and affects the oscillations of (anti-)neutrinos in different fashion. This feature invokes fake CP-asymmetry like the SM matter effect and can severely affect the leptonic CP-violation searches in long-baseline experiments. In this paper, we study in detail the possible impacts of these long-range flavor-diagonal neutral current interactions due to $L_{e}-L_{\mu}$ symmetry, when (anti-)neutrinos travel from Fermilab to Homestake $(1300 \mathrm{~km})$ and CERN to Pyhäsalmi $(2290 \mathrm{~km})$ in the context of future high-precision superbeam facilities, DUNE and LBNO respectively. If there is no signal of long-range force, DUNE (LBNO) can place stringent constraint on the effective gauge coupling $\alpha_{e \mu}<1.9 \times 10^{-53}\left(7.8 \times 10^{-54}\right)$ at $90 \%$ C.L., which is almost 30 (70) times better than the existing bound from the SuperKamiokande experiment. We also observe that if $\alpha_{e \mu} \geq 2 \times 10^{-52}$, the CP-violation discovery reach of these future facilities vanishes completely. The mass hierarchy measurement remains robust in DUNE (LBNO) if $\alpha_{e \mu}<5 \times 10^{-52}\left(10^{-52}\right)$.

Keywords: Neutrino Physics, Beyond Standard Model

ARXIV EPRINT: 1509.03517 


\section{Contents}

1 Introduction and motivation 1

2 Flavor-dependent long-range forces from gauged U(1) symmetries $\quad 3$

2.1 Abelian gauged $L_{e}-L_{\mu, \tau}$ symmetries 4

2.2 Existing phenomenological constraints on $L_{e}-L_{\mu, \tau}$ parameters 5

3 Three-flavor oscillation picture in presence of long-range potential $\quad 6$

3.1 Analytical expressions for the effective oscillation parameters 8

$\begin{array}{ll}3.2 & \text { Demonstration of the accuracy of the approximation } \\ 3.3\end{array}$

$\begin{array}{lll}3.3 & \text { Discussion at the probability level — neutrino case } & 14\end{array}$

4 Impact of long-range potential at the event level $\quad 17$

$\begin{array}{lll}\text { 4.1 Key features of DUNE and LBNO set-ups } & 17\end{array}$

$\begin{array}{lll}4.2 & \text { Event spectrum and rates } & 18\end{array}$

$\begin{array}{lll}4.3 & \text { Bi-events plot } 20\end{array}$

5 Simulation method $\quad 22$

6 Results $\quad \mathbf{2 3}$

6.1 Expected constraints on the effective gauge coupling $\alpha_{e \mu}$

$\begin{array}{ll}6.2 & \text { Discovery reach for } \alpha_{e \mu}\end{array} 25$

6.3 How robust are CP-violation searches in presence of LRF? 26

6.4 Impact of LRF on mass hierarchy measurements 30

$\begin{array}{lll}7 & \text { Summary and conclusions } & 31\end{array}$

A Discussion at the probability level - anti-neutrino case 34

\section{Introduction and motivation}

Over the past two decades or so, active attempts have been made both in experimental and theoretical fronts to improve our knowledge about neutrinos [1-3]. The three most important fundamental issues that have taken center stage in neutrino physics as a part of these activities are the following. First issue: how tiny is the neutrino mass? Second issue: can a neutrino turn into its own anti-particle? Third issue: do different neutrino flavors oscillate into one another? To shed light on the first issue, recently, the Planck Collaboration has reported an upper limit on the sum of all the neutrino mass eigenvalues of $\sum m_{i}<0.23 \mathrm{eV}$ at $95 \%$ C.L. in combination with the WMAP polarization and baryon acoustic oscillation (BAO) measurements [4]. Here, the sum runs over all the neutrino mass eigenstates which 
are in thermal equilibrium in the early Universe. As far as the second issue is concerned, the hunt for neutrinoless double beta decay process is still on which violates the total lepton number and requires Majorana neutrinos [5-8]. The third question has been answered positively only in recent years [9-11] and now, we have compelling evidence in favor of neutrino flavor oscillation $[12,13]$, suggesting that leptonic flavors are not symmetries of Nature. This entails that neutrinos are massive and they mix with each other, providing an evidence for physics beyond the Standard Model (SM) [14, 15]. This milestone has been achieved due to several fantastic world-class oscillation experiments involving neutrinos from the Sun [16-22], the Earth's atmosphere [23-25], nuclear reactors [26-36], and accelerators [37-46] which have enabled us to obtain a clear understanding of the lepton mixing pattern in three-flavor scenario. Using the standard Particle Data Group convention [14], we parametrize the vacuum Pontecorvo-Maki-Nakagawa-Sakata (PMNS) matrix $[12,47,48]$ in terms of the three mixing angles: $\theta_{12}, \theta_{23}, \theta_{13}$, and one Dirac-type $\mathrm{CP}$ phase $\delta_{\mathrm{CP}}$ (ignoring Majorana phases). In a three-flavor framework, the transition probability also depends on two independent mass-squared differences: $\Delta m_{21}^{2} \equiv m_{2}^{2}-m_{1}^{2}$ in the solar sector and $\Delta m_{32}^{2} \equiv m_{3}^{2}-m_{2}^{2}$ in the atmospheric sector where $m_{3}$ corresponds to the neutrino mass eigenstate with the smallest electron component. The smallest lepton mixing angle $\theta_{13}$ connects these two sectors and determines the impact of the sub-leading three-flavor effects [49-51]. All the neutrino oscillation data available till date have been explained quite successfully in terms of these mass-mixing parameters [52-54], excluding few anomalous results obtained at very-short-baseline experiments [55].

Neutrinos acquire additional phases while travelling in matter, the so-called 'MSW effect' [56-59] which determined the ordering of the 1-2 mass splitting using solar neutrinos. In light of large $\theta_{13}$, matter effects are also going to play an important role in presently running and future long-baseline $[49,51,60]$ neutrino oscillation experiments to settle the remaining unsolved issues, namely, the neutrino mass hierarchy, ${ }^{1}$ possibility of leptonic $\mathrm{CP}$-violation if $\delta_{\mathrm{CP}}$ differs from both $0^{\circ}$ and $180^{\circ}$, and the octant degeneracy of $\theta_{23}$ [61] if $\theta_{23}$ turns out to be non-maximal. The combined data from the current off-axis $\nu_{e}$ appearance experiments, $\mathrm{T} 2 \mathrm{~K}[62,63]$ and $\mathrm{NO} \nu \mathrm{A}[64-66]$, hold promise of providing a first hint for these missing links for only favorable ranges of oscillation parameters [67-73]. Hence, future facilities consisting of intense, high power, on-axis wide-band beams and large smart detectors have been proposed to cover the entire parameter space at unprecedented confidence level [60]. Future superbeam long-baseline facilities with liquid argon detectors, Deep Underground Neutrino Experiment (DUNE) [74-78] in the United States with a baseline of $1300 \mathrm{~km}$ from Fermilab to Homestake mine in South Dakota and Long-Baseline Neutrino Oscillation Experiment (LBNO) in Europe involving a path length of $2290 \mathrm{~km}$ between CERN and Pyhäsalmi mine in Finland [79-82] are the two major candidates in this roadmap which are capable enough to claim the discovery for the above mentioned issues $[50,51]$. For both the DUNE and LBNO baselines, the matter effects are quite significant which break the eight-fold degeneracies [83, 84] among the various oscillation parameters and improve the physics reach by considerable amount.

\footnotetext{
${ }^{1}$ Two patterns of neutrino masses are possible: $m_{3}>m_{2}>m_{1}$, called normal hierarchy (NH) where $\Delta m_{31}^{2}>0$ and $m_{2}>m_{1}>m_{3}$, called inverted hierarchy (IH) where $\Delta m_{31}^{2}<0$.
} 
Apart from the SM $W$-exchange interaction in matter, there may well be flavordependent, vector-like, leptonic long-range force (LRF), like those mediated by the $L_{e}-L_{\mu, \tau}$ gauge boson which is very light and neutral, leading to new non-universal flavor-diagonal neutral current (FDNC) interactions of the neutrinos which can give rise to non-trivial three-neutrino mixing effects in terrestrial experiments, and could affect the neutrino propagation through matter [85-89]. Can we constrain/discover these long-range FDNC interactions in upcoming long-baseline neutrino experiments? If this LRF exists in Nature, can it become fatal in our attempts to resolve the remaining unknowns in neutrino oscillation? In this paper, we attempt to address these pressing issues in the context of future high-precision superbeam facilities, DUNE and LBNO.

This paper is organized as follows. We start section 2 with a discussion on flavordependent LRF and how it arises from abelian gauged $L_{e}-L_{\mu, \tau}$ symmetry. Then, we discuss the strength of the long-range potential $V_{e \mu / e \tau}$ at the Earth surface generated by the electrons inside the Sun. This is followed by a brief discussion on the current constraints that we have on the effective gauge couplings $\alpha_{e \mu, e \tau}$ of the $L_{e}-L_{\mu, \tau}$ symmetry from various neutrino oscillation experiments. In section 3, we study in detail the three-flavor oscillation picture in presence of long-range potential. We derive the compact analytical expressions for the effective oscillation parameters in case of the $L_{e}-L_{\mu}$ symmetry, and also present a simple expression for the resonance energy, where 1-3 mixing angle in matter becomes $45^{\circ}$ in the presence of long-range potential. Next, we demonstrate the accuracy of our approximate analytical probability expressions by comparing it with the exact numerical results. We also discuss some salient features of the appearance and disappearance probabilities (calculated numerically) for the Fermilab-Homestake and CERN-Pyhäsalmi baselines in the presence of long-range potential towards the end of this section. We give the similar plots for the anti-neutrino case in appendix A. At the beginning of section 4, we give a brief description of the main experimental features of the DUNE and LBNO set-ups. Then, we study the impact of the long-range potential due to $L_{e}-L_{\mu}$ symmetry on the expected event spectra and total event rates for the DUNE and LBNO experiments. In section 5, we describe our simulation strategy. Next, we derive the expected constraints on $\alpha_{e \mu}$ from DUNE and LBNO in section 6.1, and estimate the discovery reach for $\alpha_{e \mu}$ in section 6.2. In section 6.3, we study how the long-range potential affects the CP-violation search of these future facilities. Section 6.4 is devoted to assess the impact of LRF on mass hierarchy measurements. Finally, we summarize and draw our conclusions in section 7 .

\section{Flavor-dependent long-range forces from gauged U(1) symmetries}

The SM gauge group $\mathrm{SU}(3)_{C} \times \mathrm{SU}(2)_{L} \times \mathrm{U}(1)_{Y}$ can be extended with minimal matter content by introducing anomaly free $\mathrm{U}(1)$ symmetries under which the SM remains invariant and renormalizable [90]. There are three lepton flavor combinations: $L_{e}-L_{\mu}, L_{e}-L_{\tau}$, and $L_{\mu}-L_{\tau}$ which can be gauged in an anomaly free way with the particle content of the SM [91-93]. These U(1) gauge symmetries have to be broken in Nature in order to allow the different neutrino species to mix among each other giving rise to neutrino oscillation as demanded by the recent data $[14,52-54]$. This can be understood from the following 
example. If we assume that neutrino masses are generated by effective five-dimensional operator following say, seesaw mechanism, then this operator would remain invariant under these $\mathrm{U}(1)$ symmetries if they are exact. This will ultimately give us an effective neutrino mass matrix containing a Dirac and a Majorana neutrino which remain unmixed and there will be no neutrino oscillation. For more discussions on these issues, see references [85, 88]. Now, there are two possibilities for the extra gauge boson associated with this abelian symmetry: ${ }^{2}$ either it can be very heavy or it is very light but in both the cases, it couples to matter very feebly to escape direct detection. If the electrically neutral gauge boson $\left(Z^{\prime}\right)$ corresponding to a flavor-diagonal generator of this new gauge group is relatively heavy, ${ }^{3}$ then the phenomenological consequences can be quite interesting [90-93, 100]. On the other hand, if the mass of the gauge boson is very light, then it can introduce LRF with terrestrial range (greater than or equal to the Sun-Earth distance) without call upon extremely low mass scales [85, 86]. This LRF is composition dependent (depends on the leptonic content and the mass of an object) and violate the universality of free fall which could be tested in the classic lunar ranging [101, 102] and Eötت̈os type gravity experiments $[103,104]$. In fact, this idea was given long back by Lee and Yang [105] and later, using this idea, Okun $[106,107]$ gave a $2 \sigma$ bound of $\alpha<3.4 \times 10^{-49}$ ( $\alpha$ denotes the strength of the long-range potential) for a range of the Sun-Earth distance or more. Now, the light gauge boson $Z^{\prime}$ should have a mass $m_{Z^{\prime}} \sim g\langle v\rangle$ where $g$ is the gauge coupling of the new $\mathrm{U}(1)$ symmetry and it should be $\lesssim 0.6 \times 10^{-24}$ since $g \approx \sqrt{\alpha}$ and $\langle v\rangle$ is the vacuum expectation value of the symmetry breaking scale. If the range of the LRF is comparable to the Sun-Earth distance $\left(\approx 1.5 \times 10^{13} \mathrm{~cm}\right)$ then $m_{Z^{\prime}} \sim 1 /\left(1.5 \times 10^{13} \mathrm{~cm}\right)=1.3 \times 10^{-18} \mathrm{eV}$ which means $\langle v\rangle \gtrsim 2 \mathrm{MeV}$.

\subsection{Abelian gauged $L_{e}-L_{\mu, \tau}$ symmetries}

If the extra $\mathrm{U}(1)$ corresponds to $L_{e}-L_{\mu}$ or $L_{e}-L_{\tau}$ flavor combination, ${ }^{4}$ the coupling of the solar electron to the $L_{e}-L_{\mu, \tau}$ gauge boson generates a flavor-dependent long-range potential for neutrinos [108-110] and can have significant impact on neutrino oscillations [85-89] inspite of such stringent constraints on $\alpha$. This is caused due to the fact that the $\left(L_{e}-L_{\mu, \tau}\right)$ charge of the electron neutrino is opposite to that of muon or tau flavor, leading to new non-universal FDNC interactions of the neutrinos in matter on top of the SM $W$-exchange interactions between the matter electrons and the propagating electron neutrinos. These new flavor-dependent FDNC interactions alter the 'running' of the oscillation parameters in matter by considerable amount [111]. Another important point is that the large number of electrons inside the Sun and the long-range nature of interaction balance the smallness of coupling and generate noticeable potential. As an example, the electrons inside the Sun

\footnotetext{
${ }^{2}$ Models with these symmetries necessarily possess a Higgs sector which also discriminates among different lepton families [94], but we will only focus here on the effect of the extra gauge boson.

${ }^{3}$ For an example, the $Z^{\prime}$ in gauged $L_{e}-L_{\mu, \tau}$ can be produced in $e^{+} e^{-}$collisions and subsequently decay into $e^{+} e^{-}$or $\mu^{+} \mu^{-}$or $\tau^{+} \tau^{-}$pairs and can be constrained using the data from LEP/LEP2 [95-99].

${ }^{4}$ Due to the absence of muons or tau leptons inside the Sun or Earth, we do not consider gauged $L_{\mu}-L_{\tau}$ symmetry here.
} 
give rise to a potential $V_{e \mu / e \tau}$ at the Earth surface which is given by [85, 88]

$$
V_{e \mu / e \tau}\left(R_{S E}\right)=\alpha_{e \mu / e \tau} \frac{N_{e}}{R_{S E}} \approx 1.3 \times 10^{-11} \mathrm{eV}\left(\frac{\alpha_{e \mu / e \tau}}{10^{-50}}\right),
$$

where $\alpha_{e \mu / e \tau}=\frac{g_{e \mu / e \tau}^{2}}{4 \pi}$, and $g_{e \mu / e \tau}$ is the gauge coupling of the $L_{e}-L_{\mu, \tau}$ symmetry. In eq. (2.1), $N_{e}\left(\approx 10^{57}\right)$ is the total number of electrons inside the Sun [112] and $R_{S E}$ is the Sun-Earth distance $\approx 1.5 \times 10^{13} \mathrm{~cm}=7.6 \times 10^{26} \mathrm{GeV}^{-1}$. Here $\alpha_{e \mu / e \tau}$ can be identified as the 'fine-structure constant' of the $\mathrm{U}(1)_{L_{e}-L_{\mu, \tau}}$ symmetry and its value is positive. ${ }^{5}$ The corresponding potential due to the electrons inside the Earth with a long-range force having the Earth-radius range $\left(R_{E} \sim 6400 \mathrm{~km}\right)$ is roughly one order of magnitude smaller compared to the solar long-range potential and can be safely neglected ${ }^{6}[85,88]$. The long-range potential $V_{e \mu / e \tau}$ in eq. (2.1) appears with a negative sign for anti-neutrinos and can affect the neutrino and anti-neutrino oscillation probabilities in different fashion. This feature can invoke fake CP-asymmetry like the SM matter effect and can influence the $\mathrm{CP}$-violation search in long-baseline experiments. This is one of the important findings of our paper and we will discuss this issue in detail in the later section. Now, it would be quite interesting to compare the strength of the potential given in eq. (2.1) with the quantity $\Delta m^{2} / 2 E$ which governs the neutrino oscillation probability. For long-baseline neutrinos, $\Delta m^{2} / 2 E \sim 10^{-12} \mathrm{eV}$ (assuming $\Delta m^{2} \sim 2 \times 10^{-3} \mathrm{eV}^{2}$ and $E \sim 1 \mathrm{GeV}$ ) which is comparable to $V_{e \mu / e \tau}$ even for $\alpha_{e \mu / e \tau} \sim 10^{-51}$ and can affect the long-baseline experiments significantly which we are going to explore in this paper in the context of upcoming facilities DUNE and LBNO.

\subsection{Existing phenomenological constraints on $L_{e}-L_{\mu, \tau}$ parameters}

There are phenomenological bounds on the effective gauge coupling $\alpha_{e \mu / e \tau}$ of the $L_{e}-L_{\mu, \tau}$ abelian symmetry ${ }^{7}$ using data from various neutrino oscillation experiments. It was shown in [85] that $L_{e}-L_{\mu, \tau}$ potential at the Earth due to the huge number of electrons inside the Sun suppresses the atmospheric neutrino $\nu_{\mu} \rightarrow \nu_{\tau}$ oscillations which enabled them to place tight constraints on $\alpha_{e \mu / e \tau}$ using the oscillation of multi-GeV neutrinos observed at the Super-Kamiokande (SK) experiment. They obtained an upper bound of $\alpha_{e \mu}<5.5 \times 10^{-52}$ and $\alpha_{e \tau}<6.4 \times 10^{-52}$ at $90 \%$ C.L. [85]. In [88], the authors performed a global fit to the solar neutrino and KamLAND data including the flavor-dependent LRF. They quoted an upper bound of $\alpha_{e \mu}<3.4 \times 10^{-53}$ and $\alpha_{e \tau}<2.5 \times 10^{-53}$ at $3 \sigma$ C.L. assuming $\theta_{13}=0^{\circ}$ [88]. A similar analysis was performed in [87] to place the constraints on LRF mediated by vector and non-vector (scalar or tensor) neutral bosons where the authors assumed one mass scale dominance. The proposed $50 \mathrm{kt}$ magnetized iron calorimeter (ICAL) detector at the

\footnotetext{
${ }^{5}$ In our work, we consider the case of a light vector boson exchange which makes sure that $\alpha_{e \mu / e \tau}$ is positive. It means that for an example, the force between an isolated electron and $\nu_{e}$ is repulsive.

${ }^{6}$ The possibility of the local screening of the leptonic force (generated due to the solar electrons) by the cosmic anti-neutrinos is also negligible over the Sun-Earth distance [85].

${ }^{7}$ Flavor-dependent long-range leptonic forces can also be generated via the unavoidable mixing of light $Z^{\prime}$ boson of the $L_{\mu}-L_{\tau}$ symmetry with the $Z$ boson of the SM. This issue was discussed in the context of the MINOS long-baseline experiment in [113, 114].
} 
India-based Neutrino Observatory (INO) can also probe the existence of LRF by observing the atmospheric neutrinos and anti-neutrinos separately over a wide range of energies and baselines [89]. With an exposure of one Mton.yr and using the muon momentum as observable, ICAL would be able to constrain $\alpha_{e \mu / e \tau} \lesssim 1.65 \times 10^{-53}$ at $3 \sigma$ C.L. [89].

\section{Three-flavor oscillation picture in presence of long-range potential}

In this section, we focus our attention to study the impact of flavor-dependent long-range leptonic potential (due to the electrons inside the Sun) at the Earth surface when neutrinos travel through the Earth matter. In a three-flavor framework, the long-range potential of eq. (2.1) due to $L_{e}-L_{\mu}$ symmetry modifies the effective Hamiltonian for neutrino propagation in Earth matter in the flavor basis to

$$
H_{f}=\left(U\left[\begin{array}{ccc}
0 & 0 & 0 \\
0 & \frac{\Delta m_{21}^{2}}{2 E} & 0 \\
0 & 0 & \frac{\Delta m_{31}^{2}}{2 E}
\end{array}\right] U^{\dagger}+\left[\begin{array}{ccc}
V_{C C} & 0 & 0 \\
0 & 0 & 0 \\
0 & 0 & 0
\end{array}\right]+\left[\begin{array}{ccc}
V_{e \mu} & 0 & 0 \\
0 & -V_{e \mu} & 0 \\
0 & 0 & 0
\end{array}\right]\right),
$$

where $U$ is the vacuum PMNS matrix $[12,47,48]$ which can be parametrized as

$$
U=R_{23}\left(\theta_{23}, 0\right) R_{13}\left(\theta_{13}, \delta_{\mathrm{CP}}\right) R_{12}\left(\theta_{12}, 0\right) \text {. }
$$

In the above equation, $E$ is the neutrino energy and $V_{C C}$ is the Earth matter potential which appears in the form

$$
V_{C C}=\sqrt{2} G_{F} N_{e} \simeq 7.6 \times Y_{e} \times \frac{\rho}{10^{14} \mathrm{~g} / \mathrm{cm}^{3}} \mathrm{eV},
$$

where $G_{F}$ is the Fermi coupling constant, $N_{e}$ is the electron number density inside the Earth, $\rho$ is the matter density, and $Y_{e}=\frac{N_{e}}{N_{p}+N_{n}}$ is the relative electron number density. $N_{p}, N_{n}$ are the proton and neutron densities in Earth matter respectively. In an electrically neutral, isoscalar medium, we have $N_{e}=N_{p}=N_{n}$ and $Y_{e}$ comes out to be 0.5. In eq. (3.1), $V_{e \mu}$ is the long-range potential due to $L_{e}-L_{\mu}$ symmetry and its strength is given by eq. (2.1). In case of $L_{e}-L_{\tau}$ symmetry, the contribution due to long-range potential in eq. (3.1) takes the form $\operatorname{Diag}\left(V_{e \tau}, 0,-V_{e \tau}\right)$. Note that the strength of the long-range potential $V_{e \mu / e \tau}$ does not depend on the Earth matter density ${ }^{8}$ and takes the same value for any baseline on the Earth. In case of anti-neutrino propagation, we have to reverse the sign of $V_{C C}, V_{e \mu}$ (or $\left.V_{e \tau}\right)$, and the CP phase $\delta_{\mathrm{CP}}$.

To judge the impact of the long-range potential $V_{e \mu}$ in long-baseline experiments with multi-GeV neutrinos, we need to compare its strength with the two other main terms in eq. (3.1) which are $\frac{\Delta m_{31}^{2}}{2 E}$ and the Earth matter potential $V_{C C}$. In table 1, we compare the strengths of these three quantities which control the 'running' of the oscillation parameters in matter. The first oscillation maximum for the DUNE (LBNO) experiment occurs at $2.56 \mathrm{GeV}\left(4.54 \mathrm{GeV}\right.$ ) assuming $\Delta m_{31}^{2}=2.44 \times 10^{-3} \mathrm{eV}^{2}$ (see table 2 ). In the fourth column

\footnotetext{
${ }^{8}$ This is in contrast to the usual non-standard interactions (NSI's) whose strengths are proportional to the Earth matter density.
} 


\begin{tabular}{|l|c|c|c|c|c|}
\hline \multirow{2}{*}{ Set-up } & \multirow{2}{*}{$1^{\text {st }}$ osc. max. $(\mathrm{GeV})$} & $\frac{\Delta m_{31}^{2}}{2 E}(\mathrm{eV})$ & $V_{C C}(\mathrm{eV})$ & \multicolumn{2}{|c|}{$V_{e \mu}(\mathrm{eV})$} \\
\cline { 5 - 6 } & & & & $\alpha_{e \mu}=10^{-52}$ & $\alpha_{e \mu}=10^{-53}$ \\
\hline DUNE & 2.56 & $4.8 \times 10^{-13}$ & $1.1 \times 10^{-13}$ & $1.3 \times 10^{-13}$ & $1.3 \times 10^{-14}$ \\
\hline LBNO & 4.54 & $2.7 \times 10^{-13}$ & $1.3 \times 10^{-13}$ & $1.3 \times 10^{-13}$ & $1.3 \times 10^{-14}$ \\
\hline
\end{tabular}

Table 1. The second column shows the first oscillation maxima for Fermilab-Homestake and CERN-Pyhäsalmi baselines considering $\Delta m_{31}^{2}=2.44 \times 10^{-3} \mathrm{eV}^{2}$. The fourth column depicts the strength of the Earth matter potentials for these baselines. The estimate of the long-range potentials $V_{e \mu}$ for two different values of $\alpha_{e \mu}$ is given in the last column.

of table 1 , the values of $V_{C C}$ have been estimated using eq. (3.3) where we take the lineaveraged constant Earth matter densities ${ }^{9}$ for both the baselines: $\rho=2.87 \mathrm{~g} / \mathrm{cm}^{3}$ for the DUNE baseline and $\rho=3.54 \mathrm{~g} / \mathrm{cm}^{3}$ for the LBNO baseline. Table 1 shows that around first oscillation maximum, the strengths of the terms $\frac{\Delta m_{31}^{2}}{2 E}, V_{C C}$, and $V_{e \mu}$ are comparable for both the set-ups under consideration even if $\alpha_{e \mu}$ is as small as $10^{-52}$. It means that these three terms can interfere with each other having significant impact on the oscillation probability which we are going to study in this section with the help of analytical expressions. Before we start deriving our approximate analytical expressions for the effective mass-squared differences and mixing angles in matter in the presence of longrange potential, let us take a look at the current status of the oscillation parameters. The second column of table 2 shows the present best-fit values and $1 \sigma$ errors on the three-flavor oscillation parameters assuming normal hierarchy in the fit [52]. We use the benchmark values of the various oscillation parameters as given in the third column of table 2 to draw the oscillation probability plots in this section and to generate the 'observed' data set while estimating the physics reach of the experimental set-ups. The ranges over which $\sin ^{2} \theta_{23}$ and $\delta_{\mathrm{CP}}$ are marginalized while minimizing the $\chi^{2}$ are given in the fourth column which we will discuss in detail while describing the simulation method in the later section. In table 2 , $\Delta m_{\mu \mu}^{2}$ is the effective mass-squared difference measured by the accelerator experiments using $\nu_{\mu} \rightarrow \nu_{\mu}$ disappearance channel $[41,116]$ and it is a linear combination of $\Delta m_{31}^{2}$ and $\Delta m_{21}^{2}$. The value of $\Delta m_{31}^{2}$ is estimated from $\Delta m_{\mu \mu}^{2}$ using the relation $[117,118]$

$$
\Delta m_{31}^{2}=\Delta m_{\mu \mu}^{2}+\Delta m_{21}^{2}\left(\cos ^{2} \theta_{12}-\cos \delta_{\mathrm{CP}} \sin \theta_{13} \sin 2 \theta_{12} \tan \theta_{23}\right) .
$$

The value of $\Delta m_{31}^{2}$ is calculated separately for NH and IH using the above equation assuming $\Delta m_{\mu \mu}^{2}= \pm 2.4 \times 10^{-3} \mathrm{eV}^{2}$ where positive (negative) sign is for $\mathrm{NH}(\mathrm{IH})$. Note that through out this paper, whenever we vary $\delta_{\mathrm{CP}}$ or $\theta_{23}$ or both, we calculate a new value for $\Delta m_{31}^{2}$ using eq. (3.4).

\footnotetext{
${ }^{9}$ These line-averaged constant Earth matter densities have been estimated using the Preliminary Reference Earth Model (PREM) [115].
} 


\begin{tabular}{|c|c|c|c|}
\hline Parameter & Best-fit and $1 \sigma$ error & True value & Marginalization range \\
\hline $\sin ^{2} \theta_{12}$ & $0.304_{-0.012}^{+0.013}$ & 0.304 & Not marginalized \\
\hline $\sin ^{2} \theta_{13}$ & $0.0218_{-0.0010}^{+0.0010}$ & 0.0218 & Not marginalized \\
\hline $\sin ^{2} \theta_{23}$ & $0.452_{-0.028}^{+0.052}$ & 0.50 & {$[0.38,0.64]$} \\
\hline$\delta_{\mathrm{CP}} /^{\circ}$ & $306_{-70}^{+39}$ & {$[0,360]$} & {$[0,360]$} \\
\hline$\frac{\Delta m_{21}^{2}}{10^{-5} \mathrm{eV}^{2}}$ & $7.5_{-0.17}^{+0.19}$ & 7.50 & Not marginalized \\
\hline$\frac{\Delta m_{31}^{2}}{10^{-3} \mathrm{eV}^{2}}$ & $2.457_{-0.047}^{+0.047}$ & 2.44 & Not marginalized \\
\hline$\frac{\Delta m_{\mu \mu}^{2}}{10^{-3} \mathrm{eV}^{2}}$ & $2.410_{-0.056}^{+0.051}$ & 2.40 & Not marginalized \\
\hline
\end{tabular}

Table 2. The second column shows the current best-fit values and $1 \sigma$ uncertainties on the threeflavor oscillation parameters assuming normal hierarchy in the fit [52]. The third column shows the true values of the oscillation parameters used to simulate the 'observed' data set. The fourth column depicts the range over which $\sin ^{2} \theta_{23}$ and $\delta_{\mathrm{CP}}$ are varied while minimizing the $\chi^{2}$ to obtain the final results. In our calculations, $\Delta m_{\mu \mu}^{2}$ serves as an input parameter and then we estimate the value of $\Delta m_{31}^{2}$ using the relation given in eq. (3.4) (see text for details). In the third column, we take $\delta_{\mathrm{CP}}=0^{\circ}$ to calculate the value of $\Delta m_{31}^{2}$ from $\Delta m_{\mu \mu}^{2}$.

\subsection{Analytical expressions for the effective oscillation parameters}

In a CP-conserving scenario $\left(\delta_{\mathrm{CP}}=0^{\circ}\right)$, the effective Hamiltonian in the flavor basis given in eq. (3.1) takes the form

$$
H_{f}=R_{23}\left(\theta_{23}\right) R_{13}\left(\theta_{13}\right) R_{12}\left(\theta_{12}\right) H_{0} R_{12}^{T}\left(\theta_{12}\right) R_{13}^{T}\left(\theta_{13}\right) R_{23}^{T}\left(\theta_{23}\right)+V,
$$

where $H_{0}=\operatorname{Diag}\left(0, \Delta_{21}, \Delta_{31}\right)$ with $\Delta_{21} \equiv \Delta m_{21}^{2} / 2 E$ and $\Delta_{31} \equiv \Delta m_{31}^{2} / 2 E$. In the above equation, $V=\operatorname{Diag}\left(V_{C C}+V_{e \mu},-V_{e \mu}, 0\right)$ for $L_{e}-L_{\mu}$ symmetry. We can rewrite $H_{f}$ in eq. (3.5) as

$$
H_{f}=\Delta_{31}\left(\begin{array}{lll}
a_{11} & a_{12} & a_{13} \\
a_{12} & a_{22} & a_{23} \\
a_{13} & a_{23} & a_{33}
\end{array}\right)
$$

where

$$
\begin{aligned}
& a_{11}=A+W+\sin ^{2} \theta_{13}+\alpha \cos ^{2} \theta_{13} \sin ^{2} \theta_{12}, \\
& a_{12}=\frac{1}{\sqrt{2}}\left[\cos \theta_{13}\left(\alpha \cos \theta_{12} \sin \theta_{12}+\sin \theta_{13}-\alpha \sin ^{2} \theta_{12} \sin \theta_{13}\right)\right], \\
& a_{13}=\frac{1}{\sqrt{2}}\left[\cos \theta_{13}\left(-\alpha \cos \theta_{12} \sin \theta_{12}+\sin \theta_{13}-\alpha \sin ^{2} \theta_{12} \sin \theta_{13}\right)\right], \\
& a_{22}=\frac{1}{2}\left[\alpha \cos ^{2} \theta_{12}+\cos ^{2} \theta_{13}-2 \alpha \cos \theta_{12} \sin \theta_{12} \sin \theta_{13}+\alpha \sin ^{2} \theta_{12} \sin ^{2} \theta_{13}-2 W\right], \\
& a_{23}=\frac{1}{2}\left[\cos ^{2} \theta_{13}-\alpha \cos ^{2} \theta_{12}+\alpha \sin ^{2} \theta_{12} \sin ^{2} \theta_{13}\right], \\
& a_{33}=\frac{1}{2}\left[\cos ^{2} \theta_{13}+\alpha \cos ^{2} \theta_{12}+\alpha \sin \theta_{13}\left(\sin 2 \theta_{12}+\sin ^{2} \theta_{12} \sin \theta_{13}\right)\right] .
\end{aligned}
$$


In the above equations, we introduce the terms $A, W$, and $\alpha$ which are defined as

$$
A \equiv \frac{V_{C C}}{\Delta_{31}}=\frac{2 E V_{C C}}{\Delta m_{31}^{2}}, W \equiv \frac{V_{e \mu}}{\Delta_{31}}=\frac{2 E V_{e \mu}}{\Delta m_{31}^{2}}, \alpha \equiv \frac{\Delta m_{21}^{2}}{\Delta m_{31}^{2}},
$$

and we assume that the vacuum value of $\theta_{23}$ is $45^{\circ}$. Note that we have kept the terms of all orders in $\sin \theta_{13}$ and $\alpha$ which are quite important in light of the large value of 1-3 mixing angle as was measured recently by the modern reactor experiments. Now, we need to diagonalize the effective Hamiltonian $H_{f}$ in eq. (3.6) to find the effective mass-squared differences and mixing angles in the presence of the Earth matter potential $\left(V_{C C}\right)$ and the long-range potential $\left(V_{e \mu}\right)$. We can almost diagonalize $H_{f}$ with the help of a unitary matrix

$$
\tilde{U} \equiv R_{23}\left(\theta_{23}^{m}\right) R_{13}\left(\theta_{13}^{m}\right) R_{12}\left(\theta_{12}^{m}\right)
$$

such that

$$
\tilde{U}^{T} H_{f} \tilde{U} \simeq \operatorname{Diag}\left(m_{1, m}^{2} / 2 E, m_{2, m}^{2} / 2 E, m_{3, m}^{2} / 2 E\right),
$$

where off-diagonal terms are quite small and can be safely neglected. The lower right $2 \times 2$ block in eq. (3.6) gives us the angle $\theta_{23}^{m}$ which has the form

$$
\tan 2 \theta_{23}^{m}=\frac{\cos ^{2} \theta_{13}-\alpha \cos ^{2} \theta_{12}+\alpha \sin ^{2} \theta_{12} \sin ^{2} \theta_{13}}{W+\alpha \sin 2 \theta_{12} \sin \theta_{13}} .
$$

The mixing angles $\theta_{13}^{m}$ and $\theta_{12}^{m}$ can be obtained by subsequent diagonalizations of the $(1,3)$ and $(1,2)$ blocks respectively and we get the following expressions

$$
\tan 2 \theta_{13}^{m}=\frac{\sin 2 \theta_{13}\left(1-\alpha \sin ^{2} \theta_{12}\right)\left(\cos \theta_{23}^{m}+\sin \theta_{23}^{m}\right)-\alpha \sin 2 \theta_{12} \cos \theta_{13}\left(\cos \theta_{23}^{m}-\sin \theta_{23}^{m}\right)}{\sqrt{2}\left(\lambda_{3}-A-W-\sin ^{2} \theta_{13}-\alpha \sin ^{2} \theta_{12} \cos ^{2} \theta_{13}\right)},
$$

and

$$
\begin{aligned}
& \tan 2 \theta_{12}^{m}= \\
& \frac{\cos \theta_{13}^{m}\left[\sin 2 \theta_{13}\left(1-\alpha \sin ^{2} \theta_{12}\right)\left(\cos \theta_{23}^{m}-\sin \theta_{23}^{m}\right)+\alpha \sin 2 \theta_{12} \cos \theta_{13}\left(\cos \theta_{23}^{m}+\sin \theta_{23}^{m}\right)\right]}{\sqrt{2}\left(\lambda_{2}-\lambda_{1}\right)},
\end{aligned}
$$

where

$$
\begin{aligned}
& \lambda_{3}=\frac{1}{2}\left[\cos ^{2} \theta_{13}+\alpha \cos ^{2} \theta_{12}+\alpha \sin ^{2} \theta_{12} \sin ^{2} \theta_{13}-W+\frac{W+\alpha \sin 2 \theta_{12} \sin \theta_{13}}{\cos 2 \theta_{23}^{m}}\right], \\
& \lambda_{2}=\frac{1}{2}\left[\cos ^{2} \theta_{13}+\alpha \cos ^{2} \theta_{12}+\alpha \sin ^{2} \theta_{12} \sin ^{2} \theta_{13}-W-\frac{W+\alpha \sin 2 \theta_{12} \sin \theta_{13}}{\cos 2 \theta_{23}^{m}}\right],
\end{aligned}
$$

and

$$
\begin{aligned}
\lambda_{1}= & \frac{1}{2}\left[\left(\lambda_{3}+A+W+\sin ^{2} \theta_{13}+\alpha \cos ^{2} \theta_{13} \sin ^{2} \theta_{12}\right)\right. \\
& \left.-\frac{\left(\lambda_{3}-A-W-\sin ^{2} \theta_{13}-\alpha \cos ^{2} \theta_{13} \sin ^{2} \theta_{12}\right)}{\cos 2 \theta_{13}^{m}}\right] .
\end{aligned}
$$



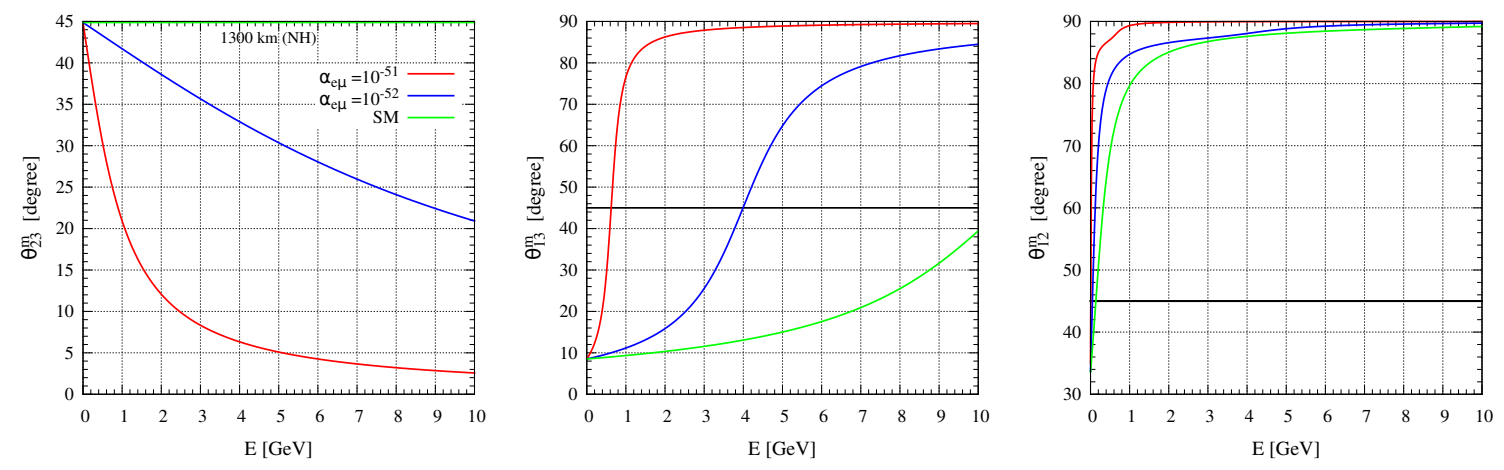

Figure 1. The variations in the effective mixing angles with the neutrino energy $E$ in the presence of the Earth matter potential $\left(V_{C C}\right)$ and long-range potential $\left(V_{e \mu}\right)$. The left, middle, and right panels show the 'running' of $\theta_{23}^{m}, \theta_{13}^{m}$, and $\theta_{12}^{m}$ respectively. Here, we take $L=1300 \mathrm{~km}$ which corresponds to the Fermilab-Homestake baseline and assume NH. Plots are given for three different choices of the effective gauge coupling $\alpha_{e \mu}$ : 0 (the SM case), $10^{-52}$, and $10^{-51}$. The vacuum values of the oscillation parameters are taken from the third column of table 2 and we consider $\delta_{\mathrm{CP}}=0^{\circ}$.

The eigenvalues $m_{i, m}^{2} / 2 E(i=1,2,3)$ are given by the expressions

$$
\begin{aligned}
m_{3, m}^{2} / 2 E= & \frac{\Delta_{31}}{2}\left[\left(\lambda_{3}+A+W+\sin ^{2} \theta_{13}+\alpha \cos ^{2} \theta_{13} \sin ^{2} \theta_{12}\right)\right. \\
& \left.+\frac{\left(\lambda_{3}-A-W-\sin ^{2} \theta_{13}-\alpha \cos ^{2} \theta_{13} \sin ^{2} \theta_{12}\right)}{\cos 2 \theta_{13}^{m}}\right], \\
m_{2, m}^{2} / 2 E= & \frac{\Delta_{31}}{2}\left[\lambda_{1}+\lambda_{2}-\frac{\left(\lambda_{1}-\lambda_{2}\right)}{\cos 2 \theta_{12}^{m}}\right],
\end{aligned}
$$

and

$$
m_{1, m}^{2} / 2 E=\frac{\Delta_{31}}{2}\left[\lambda_{1}+\lambda_{2}+\frac{\left(\lambda_{1}-\lambda_{2}\right)}{\cos 2 \theta_{12}^{m}}\right] .
$$

With the help of eq. (3.16), eq. (3.17), and eq. (3.18), we plot the 'running' of the effective mixing angles $\theta_{23}^{m}, \theta_{13}^{m}$, and $\theta_{12}^{m}$ respectively in figure 1 as functions of the neutrino energy $E$ in the presence of $V_{C C}$ and $V_{e \mu}$. Here, we consider $L=1300 \mathrm{~km}$ and NH. We give the plots for three different choices of the effective gauge coupling $\alpha_{e \mu}$ : 0 (the SM case), $10^{-52}$, and $10^{-51}$. The vacuum values of the oscillation parameters are taken from the third column of table 2. We do the same for the effective mass-squared differences (see eqs. (3.22), (3.23), and (3.24)) in figure 2. The extreme right panel of figure 1 shows that $\theta_{12}^{m}$ approaches to $90^{\circ}$ very rapidly with increasing $E$ in the presence of $V_{C C}$ and this behavior does not change much when we introduce $V_{e \mu}$. This is not the case for $\theta_{23}^{m}$ and $\theta_{13}^{m}$. The long-range potential $V_{e \mu}$ affects the 'running' of $\theta_{23}^{m}$ (see extreme left panel of figure 1) significantly. As we go to higher energies, $\theta_{23}^{m}$ deviates from $45^{\circ}$ and its value decreases very sharply depending on the strength of $\alpha_{e \mu}$. In case of $\theta_{13}^{m}$, the effect of $V_{e \mu}$ is quite opposite as compared to $\theta_{23}^{m}$ as can be seen from the middle panel of figure 1. Assuming $\mathrm{NH}$, as we increase $E, \theta_{13}^{m}$ quickly reaches to $45^{\circ}$ (resonance point) in the presence of $V_{e \mu}$ and then finally approaches toward $90^{\circ}$ as we further increase $E$. These two opposite 

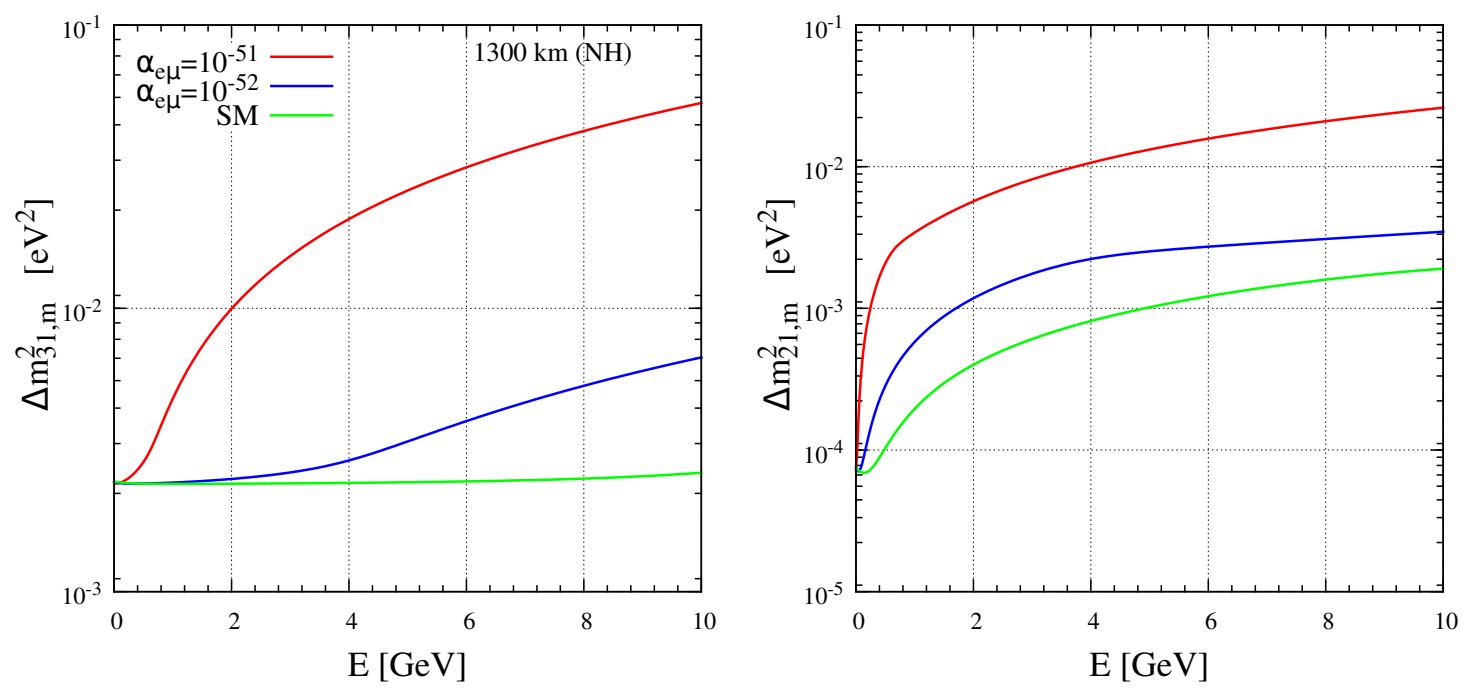

Figure 2. The variations in the effective mass-squared differences with the neutrino energy $E$ in the presence of $V_{C C}$ and $V_{e \mu}$. Left panel shows the 'running' of $\Delta m_{31, m}^{2}\left(\equiv m_{3, m}^{2}-m_{1, m}^{2}\right)$ while right panel is for $\Delta m_{21, m}^{2}\left(\equiv m_{2, m}^{2}-m_{1, m}^{2}\right)$. Here, we take $L=1300 \mathrm{~km}$ and assume NH. We give plots for three different choices of the effective gauge coupling $\alpha_{e \mu}$ : 0 (the SM case), $10^{-52}$, and $10^{-51}$. The vacuum values of the oscillation parameters are taken from the third column of table 2 and we consider $\delta_{\mathrm{CP}}=0^{\circ}$.

behaviors of $\theta_{23}^{m}$ and $\theta_{13}^{m}$ alter the amplitudes and the locations of oscillation maxima in the transition probability substantially for non-zero $\alpha_{e \mu}$ which we discuss in the later part of this section. For $\alpha_{e \mu}=10^{-52}\left(10^{-51}\right)$, the resonance occurs around $4 \mathrm{GeV}(0.6 \mathrm{GeV})$ for $1300 \mathrm{~km}$ baseline. We can obtain an analytical expression for the resonance energy demanding $\theta_{13}^{m}=45^{\circ}$ in eq. (3.17). In one mass scale dominance approximation where $\Delta m_{21}^{2}$ can be neglected i.e. assuming $\alpha=0$, the condition for the resonance energy $\left(E_{\text {res }}\right)$ takes the form:

$$
\lambda_{3}=A+W+\sin ^{2} \theta_{13} .
$$

Now, putting $\alpha=0$ in eqs. (3.19) and (3.16), we get a simplified expression for $\lambda_{3}$ which has the following form

$$
\lambda_{3}=\frac{1}{2}\left[\cos ^{2} \theta_{13}-W+\sqrt{W^{2}+\left(\cos ^{2} \theta_{13}\right)^{2}}\right] \simeq \frac{1}{2}\left[2 \cos ^{2} \theta_{13}-W\right],
$$

since at resonance energies, the term $W^{2}$ is small compared to $\cos ^{4} \theta_{13}$ and can be safely neglected. Now, comparing eq. (3.25) and eq. (3.26), we get a simple and compact expression for the resonance energy

$$
E_{\mathrm{res}}=\frac{\Delta m_{31}^{2} \cos 2 \theta_{13}}{2 V_{c c}+3 V_{e \mu}}
$$

In the absence of long-range potential $V_{e \mu}$, eq. (3.27) gives us the standard expression for the resonance energy in the SM framework. Eq. (3.27) suggests that for a given baseline, in the presence of $V_{e \mu}$, the resonance occurs at lower energy as compared to the SM case and this is exactly what we observe in the middle panel of figure 1 . The right panel of figure 2 
demonstrates that the effective solar mass-squared difference $\Delta m_{21, m}^{2}$ increases with energy and can be comparable to the vacuum value of $\Delta m_{31}^{2}$ at higher energies in the SM case. In the presence of $V_{e \mu}, \Delta m_{21, m}^{2}$ increases with energy even faster and can become quite large at higher energies depending on the strength of $\alpha_{e \mu}$. In the SM framework, the effective atmospheric mass-squared difference $\Delta m_{31, m}^{2}$ does not run with energy for this choice of baseline (see left panel of figure 2). But, in the presence of $V_{e \mu}$, the value of $\Delta m_{31, m}^{2}$ enhances a lot depending on the strength of $\alpha_{e \mu}$ as energy is increased. Next, to check the accuracy of our approximate analytical results, we compare the oscillation probabilities calculated with our approximate effective running mixing angles and mass-squared differences with those calculated numerically for the same baseline and line-averaged constant matter density along it.

\subsection{Demonstration of the accuracy of the approximation}

The neutrino oscillation probabilities in the presence of $V_{C C}$ and $V_{e \mu}$ can be obtained by replacing the vacuum expressions of the elements of the mixing matrix $U$ and the masssquare differences $\Delta m_{i j}^{2}$ with their effective 'running' values

$$
U_{\alpha i} \rightarrow \tilde{U}_{\alpha i}\left(\theta_{12} \rightarrow \theta_{12}^{m}, \theta_{13} \rightarrow \theta_{13}^{m}, \theta_{23} \rightarrow \theta_{23}^{m}\right), \Delta m_{i j}^{2} \rightarrow \Delta m_{i j, m}^{2}=m_{i, m}^{2}-m_{j, m}^{2} .
$$

Incorporating the modifications due to $V_{C C}$ and $V_{e \mu}$, the new transition probability in a $\mathrm{CP}$-conserving scenario can be written as

$$
P\left(\nu_{\alpha} \rightarrow \nu_{\beta}\right)=\delta_{\alpha \beta}-4 \sum_{i>j} \tilde{U}_{\alpha i} \tilde{U}_{\beta i} \tilde{U}_{\alpha j} \tilde{U}_{\beta j} \sin ^{2}\left(\frac{\Delta m_{i j, m}^{2} L}{4 E}\right) .
$$

Using eq. (3.29), we obtain the following expressions for the appearance and disappearance channels [119]

$$
\begin{aligned}
P\left(\nu_{\mu} \rightarrow \nu_{e}\right)= & 4 \tilde{U}_{\mu 2}^{2} \tilde{U}_{e 2}^{2} \sin ^{2} \frac{\Delta m_{21, m}^{2} L}{4 E}+4 \tilde{U}_{\mu 3}^{2} \tilde{U}_{e 3}^{2} \sin ^{2} \frac{\Delta m_{31, m}^{2} L}{4 E} \\
& +2 \tilde{U}_{\mu 3} \tilde{U}_{e 3} \tilde{U}_{\mu 2} \tilde{U}_{e 2}\left(4 \sin ^{2} \frac{\Delta m_{21, m}^{2} L}{4 E} \sin ^{2} \frac{\Delta m_{31, m}^{2} L}{4 E}\right) \\
& +2 \tilde{U}_{\mu 3} \tilde{U}_{e 3} \tilde{U}_{\mu 2} \tilde{U}_{e 2}\left(\sin \frac{\Delta m_{21, m}^{2} L}{2 E} \sin \frac{\Delta m_{31, m}^{2} L}{2 E}\right) \\
P\left(\nu_{\mu} \rightarrow \nu_{\mu}\right)= & 1-4 \tilde{U}_{\mu 2}^{2}\left(1-\tilde{U}_{\mu 2}^{2}\right) \sin ^{2} \frac{\Delta m_{21, m}^{2} L}{4 E}-4 \tilde{U}_{\mu 3}^{2}\left(1-\tilde{U}_{\mu 3}^{2}\right) \sin ^{2} \frac{\Delta m_{31, m}^{2} L}{4 E} \\
& +2 \tilde{U}_{\mu 2}^{2} \tilde{U}_{\mu 3}^{2}\left(4 \sin ^{2} \frac{\Delta m_{21, m}^{2} L}{4 E} \sin ^{2} \frac{\Delta m_{31, m}^{2} L}{4 E}\right) \\
& +2 \tilde{U}_{\mu 2}^{2} \tilde{U}_{\mu 3}^{2}\left(\sin \frac{\Delta m_{21, m}^{2} L}{2 E} \sin \frac{\Delta m_{31, m}^{2} L}{2 E}\right)
\end{aligned}
$$

In figure 3, we present our approximate $\nu_{\mu} \rightarrow \nu_{e}$ oscillation probabilities (dashed curves) as a function of the neutrino energy against the exact numerical results (solid curves) considering $L=1300 \mathrm{~km}$ (left panels) and $2290 \mathrm{~km}$ (right panels). We give the 

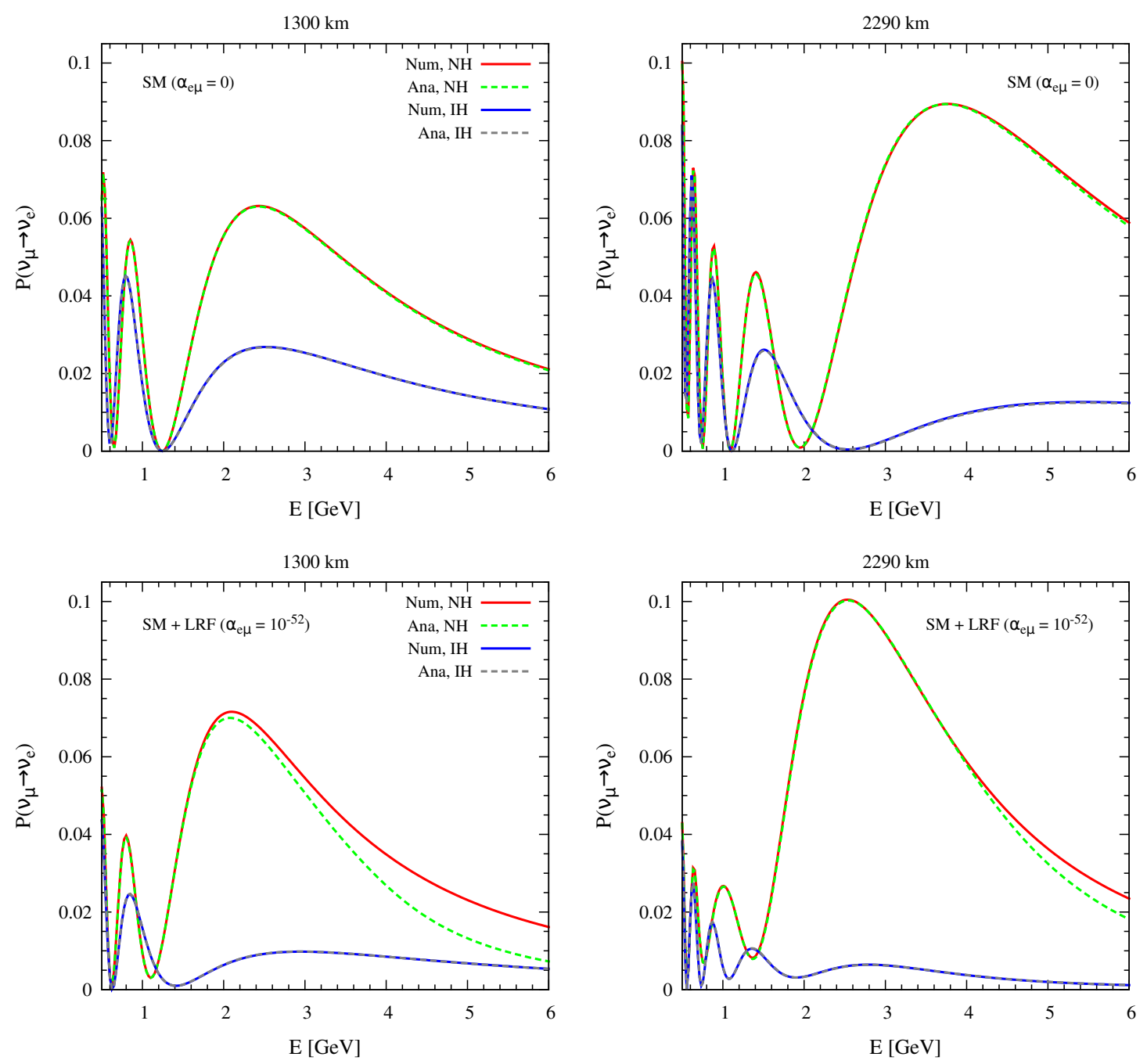

Figure 3. $\nu_{\mu} \rightarrow \nu_{e}$ transition probability as a function of neutrino energy $E$ in $\mathrm{GeV}$ for $1300 \mathrm{~km}$ $(2290 \mathrm{~km})$ baseline in left (right) panels. The upper panels are for the SM case without longrange potential. The lower panels correspond to $\alpha_{e \mu}=10^{-52}$. In all the panels, we compare our analytical expressions (dashed curves) to the exact numerical results (solid curves) for $\mathrm{NH}$ and $\mathrm{IH}$. The vacuum values of the oscillation parameters are taken from the third column of table 2 and we take $\delta_{\mathrm{CP}}=0^{\circ}$.

plots for both $\mathrm{NH}$ and $\mathrm{IH}$ in all the panels considering line-averaged constant Earth matter densities for both the baselines. The upper panels are drawn for the SM case $\left(\alpha_{e \mu}=0\right)$ where our approximate results match exactly with the numerically obtained probabilities. In the lower panels, we give the probabilities considering $\alpha_{e \mu}=10^{-52}$ and find that our approximate expressions work quite well in the presence of long-range potential and can predict almost accurate $L / E$ patterns of the oscillation probability. In figure 4 , we study the same for $\nu_{\mu} \rightarrow \nu_{\mu}$ oscillation channel and find that our approximate expressions match quite nicely with the numerical results. Here, we present our analytical results for NH. We can obtain the same for IH by changing $\Delta m_{31}^{2} \rightarrow-\Delta m_{31}^{2}$. Following the same procedure 

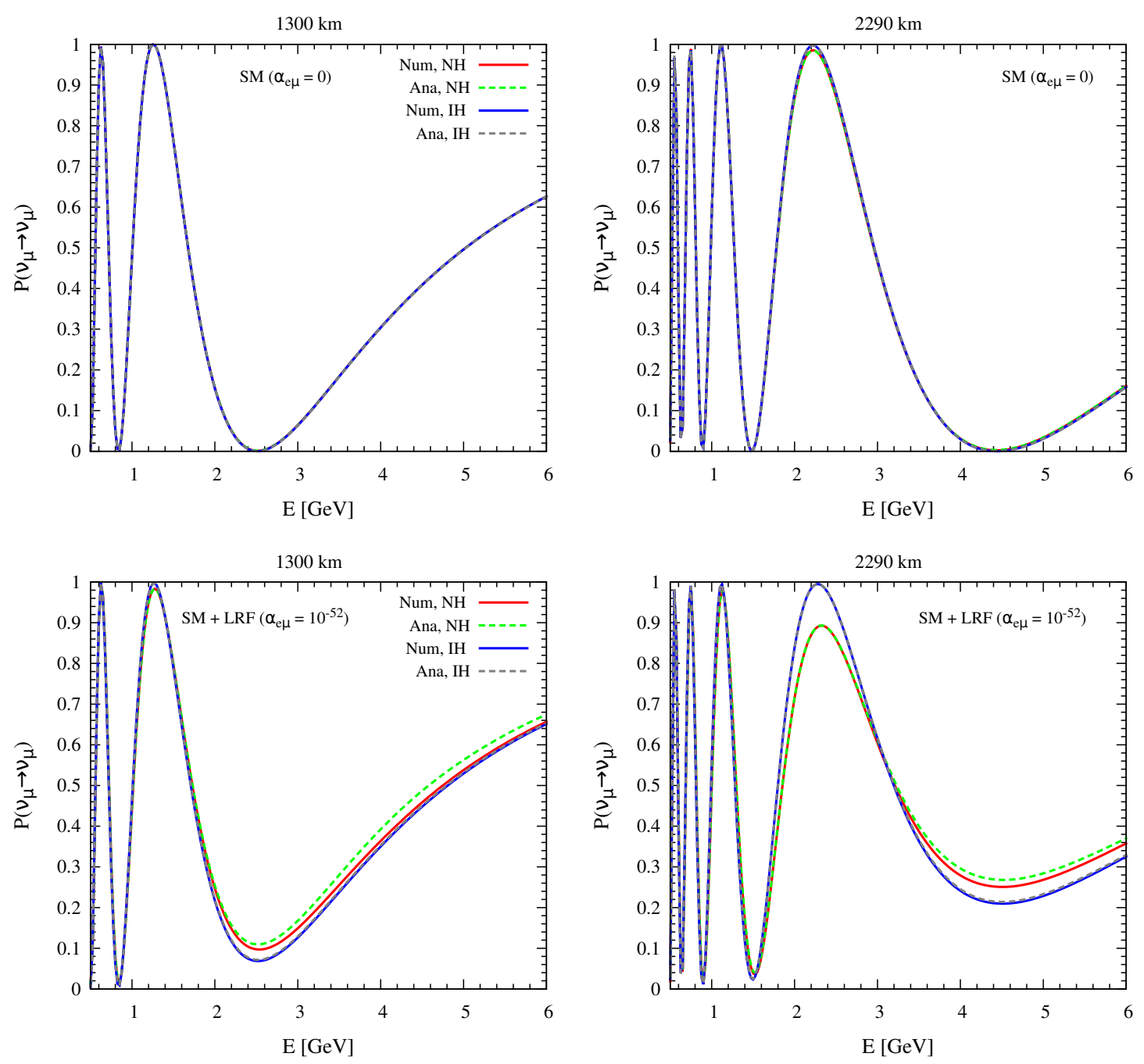

Figure 4. $\nu_{\mu} \rightarrow \nu_{\mu}$ transition probability as a function of neutrino energy $E$ in $\mathrm{GeV}$ for $1300 \mathrm{~km}$ $(2290 \mathrm{~km})$ baseline in left (right) panels. The upper panels are for the SM case without longrange potential. The lower panels correspond to $\alpha_{e \mu}=10^{-52}$. In all the panels, we compare our analytical expressions (dashed curves) to the exact numerical results (solid curves) for $\mathrm{NH}$ and $\mathrm{IH}$. The vacuum values of the oscillation parameters are taken from the third column of table 2 and we take $\delta_{\mathrm{CP}}=0^{\circ}$.

and reversing the sign of $V$ in eq. (3.5), we can derive the analytical expressions for antineutrino as well. Note that in this paper, we limit our investigation to $L_{e}-L_{\mu}$ symmetry, though similar procedure can be adopted for $L_{e}-L_{\tau}$ symmetry.

\subsection{Discussion at the probability level - neutrino case}

In this section, we discuss in detail how the long-range potential affects the full threeflavor neutrino oscillation probabilities in matter considering non-zero values of $\delta_{\mathrm{CP}}$. In figure 5, we show the exact numerical transition probability $P_{\mu e}$ as a function of the neutrino energy using the line-averaged constant Earth matter densities for $1300 \mathrm{~km}$ (left panels) 

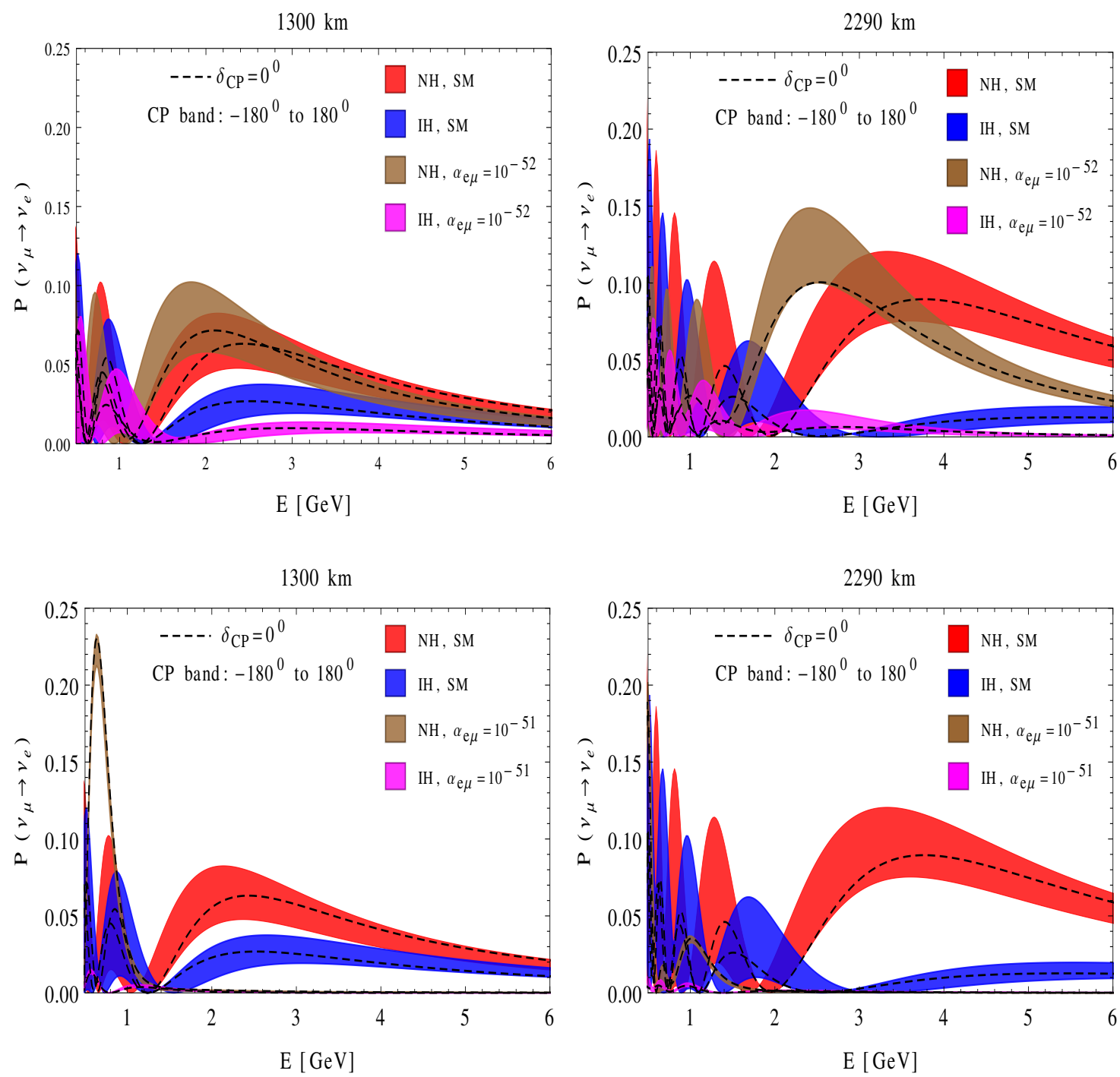

Figure 5. The transition probability $P_{\mu e}$ as a function of neutrino energy. The band reflects the effect of unknown $\delta_{\mathrm{CP}}$. Inside each band, the probability for $\delta_{\mathrm{CP}}=0^{\circ}$ case is shown by the black dashed line. The left panels (right panels) are for $1300 \mathrm{~km}(2290 \mathrm{~km})$ baseline. In each panel, we compare the probabilities for $\mathrm{NH}$ and $\mathrm{IH}$ with and without long-range potential. In the upper (lower) panels, we take $\alpha_{e \mu}=10^{-52}\left(\alpha_{e \mu}=10^{-51}\right)$ for the cases with long-range potential.

and $2290 \mathrm{~km}$ (right panels) baselines. We vary $\delta_{\mathrm{CP}}$ within the range $-180^{\circ}$ to $180^{\circ}$ and the resultant probability is shown as a band, with the thickness of the band reflecting the effect of $\delta_{\mathrm{CP}}$ on $P_{\mu e}$. Inside each band, the probability for $\delta_{\mathrm{CP}}=0^{\circ}$ case is shown explicitly by the black dashed line. The left panels (right panels) are for $1300 \mathrm{~km}(2290 \mathrm{~km})$ baseline. In each panel, we compare the probabilities for $\mathrm{NH}$ and $\mathrm{IH}$ with and without long-range potential. In the upper (lower) panels, we take $\alpha_{e \mu}=10^{-52}\left(\alpha_{e \mu}=10^{-51}\right)$ for the cases with long-range potential. We study the same for the disappearance $\left(\nu_{\mu} \rightarrow \nu_{\mu}\right)$ channel in figure 6 . We give the similar plots for the anti-neutrino case in appendix A. 

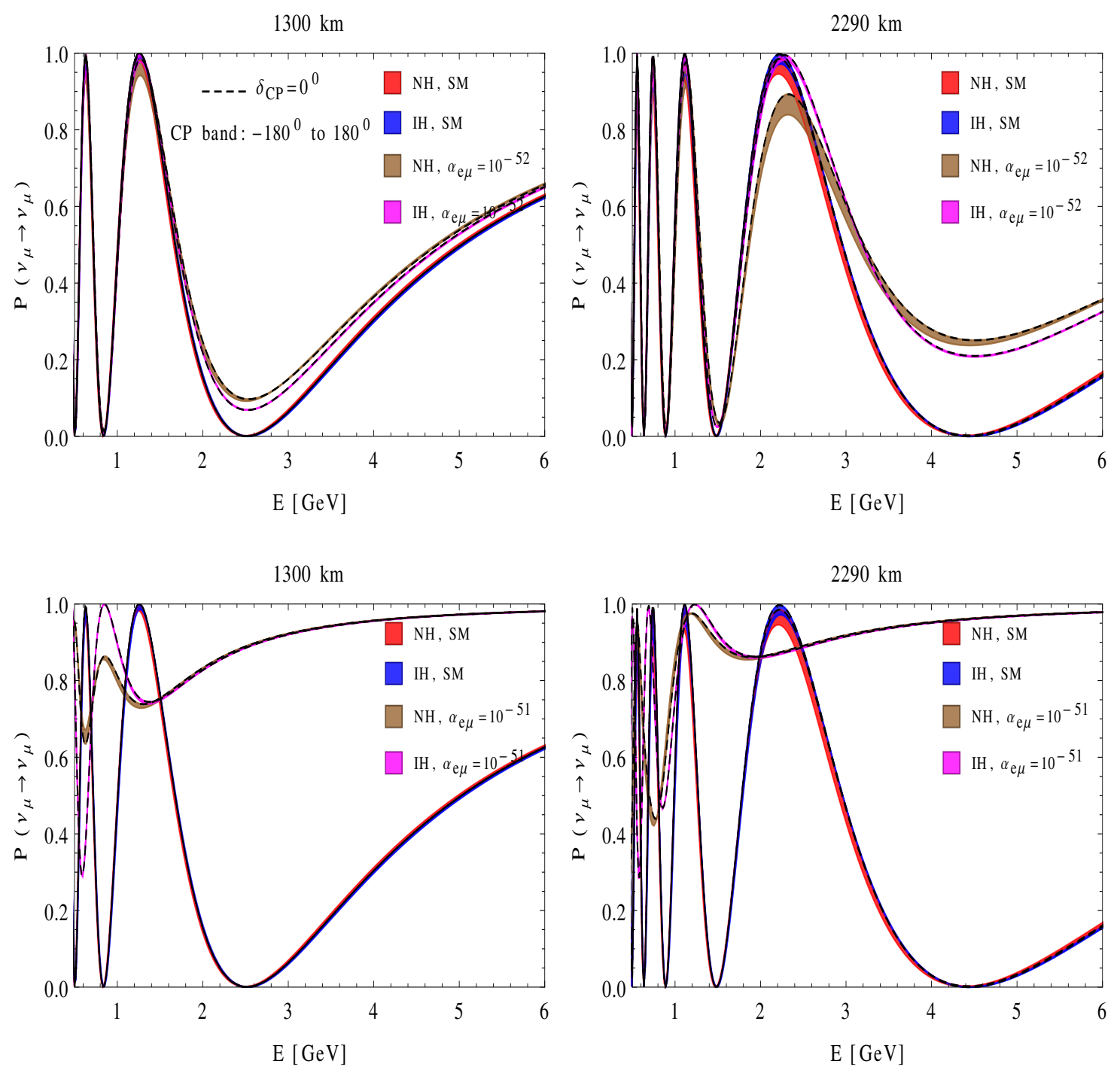

Figure 6. The transition probability $P_{\mu \mu}$ as a function of neutrino energy. The band reflects the effect of unknown $\delta_{\mathrm{CP}}$. Inside each band, the probability for $\delta_{\mathrm{CP}}=0^{\circ}$ case is shown by the black dashed line. The left panels (right panels) are for $1300 \mathrm{~km}(2290 \mathrm{~km})$ baseline. In each panel, we compare the probabilities for $\mathrm{NH}$ and $\mathrm{IH}$ with and without long-range potential. In the upper (lower) panels, we take $\alpha_{e \mu}=10^{-52}\left(\alpha_{e \mu}=10^{-51}\right)$ for the cases with long-range potential.

To explain the behavior of the oscillation probabilities in figure 5 and figure 6 at the qualitative level, we can simplify the analytical expressions given in eqs. (3.30) and (3.31) in the following fashion. The extreme right panel of figure 1 suggests that $\sin \theta_{12}^{m} \rightarrow 1$ and $\cos \theta_{12}^{m} \rightarrow 0$ very quickly as we increase $E$ in the SM case or with non-zero $\alpha_{e \mu}$. So, we set $\sin \theta_{12}^{m} \approx 1$ and $\cos \theta_{12}^{m} \approx 0$ in eqs. (3.30) and (3.31) and obtain the following simple expressions:

$$
P\left(\nu_{\mu} \rightarrow \nu_{e}\right)=\sin ^{2} \theta_{23}^{m} \sin ^{2} 2 \theta_{13}^{m} \sin ^{2} \frac{\Delta m_{32, m}^{2} L}{4 E},
$$


and

$$
\begin{aligned}
P\left(\nu_{\mu} \rightarrow \nu_{\mu}\right)= & 1-\sin ^{2} 2 \theta_{23}^{m} \sin ^{2} \theta_{13}^{m} \sin ^{2} \frac{\Delta m_{21, m}^{2} L}{4 E} \\
& -\sin ^{2} 2 \theta_{23}^{m} \cos ^{2} \theta_{13}^{m} \sin ^{2} \frac{\Delta m_{31, m}^{2} L}{4 E} \\
& -\sin ^{4} \theta_{23}^{m} \sin ^{2} 2 \theta_{13}^{m} \sin ^{2} \frac{\Delta m_{32, m}^{2} L}{4 E} .
\end{aligned}
$$

In figure 5 , we can see that for $\alpha_{e \mu}=10^{-52}$ case (upper panels), the locations of the first oscillation maxima have been shifted toward lower energies for both the baselines and also the amplitudes of the first oscillation maxima have been enhanced when we assume NH. This can be understood from the 'running' of $\theta_{23}^{m}, \theta_{13}^{m}$ (extreme left and middle panels of figure 1), and $\Delta m_{32, m}^{2}$ (left and right panels of figure 2). As we go to higher energies, $\theta_{13}^{m}$ increases and $\theta_{23}^{m}$ decreases and there is a trade-off between the terms $\sin ^{2} \theta_{23}^{m}$ and $\sin ^{2} 2 \theta_{13}^{m}$ in eq. (3.32). Also, the value of $\Delta m_{32, m}^{2}\left(\Delta m_{31, m}^{2}-\Delta m_{21, m}^{2}\right)$ decreases with energy as $\Delta m_{21, m}^{2}$ increases by substantial amount compared to $\Delta m_{31, m}^{2}$, which shifts the location of the first oscillation maxima toward lower energies. For IH, the value of $\theta_{13}^{m}$ decreases fast with non-zero $\alpha_{e \mu}$ compared to the SM case, causing a depletion in the probabilities over a wide range of energies. In case of $\alpha_{e \mu}=10^{-51}$ (lower panels), there is a huge suppression in the probabilities at both the baselines over a wide range of energies above $1 \mathrm{GeV}$ assuming

$\mathrm{NH}$. The main reason behind this large damping in the probabilities is that $\theta_{13}^{m}$ approaches very quickly to $90^{\circ}$ around $1 \mathrm{GeV}$ or so for $\alpha_{e \mu}=10^{-51}$ (see middle panel of figure 1) and therefore, $\sin ^{2} 2 \theta_{13}^{m} \rightarrow 0$, vanishing the probability amplitude for $\nu_{\mu} \rightarrow \nu_{e}$ oscillation channel. Below $1 \mathrm{GeV}, \theta_{13}^{m}$ runs toward $45^{\circ}$ and therefore, $\sin ^{2} 2 \theta_{13}^{m} \rightarrow 1$, causing the enhancement in the probabilities. When we take IH, $\theta_{13}^{m}$ quickly advances to zero, causing a huge damping in the probabilities at all the energies. These 'running' behaviors of $\theta_{23}^{m}$, $\theta_{13}^{m}$, and the mass-squared differences in the presence of long-range potential as discussed above also affect $\nu_{\mu} \rightarrow \nu_{\mu}$ oscillation channel (see figure 6) which can be explained with the help of eq. (3.33). Next, we discuss how the long-range potential due to $L_{e}-L_{\mu}$ symmetry modifies the expected event spectra and total event rates of the DUNE and LBNO experiments.

\section{Impact of long-range potential at the event level}

We start this section with a brief description of the main experimental features of the DUNE and LBNO set-ups that we use in our simulation.

\subsection{Key features of DUNE and LBNO set-ups}

The proposed DUNE experiment $[74-78,120]$ in the United States with a baseline of $1300 \mathrm{~km}$ from Fermilab to Homestake mine in South Dakota is planning to build a massive $35 \mathrm{kt}$ liquid argon time projection chamber (LArTPC) as the far detector choice. This LArTPC will have excellent kinematic reconstruction capability for all the observed particles, rejecting almost all of the large neutral current background. We use the detector properties which are given in table 1 of ref. [79]. As far as the neutrinos are concerned, this 
facility will have a new, high intensity, on-axis neutrino beam, which in its initial phase, will operate at a proton beam power of $708 \mathrm{~kW}$, with proton energy of $120 \mathrm{GeV}$, delivering $6 \times 10^{20}$ protons on target in 230 days per calendar year. In this work, we have used the latest fluxes being considered by the collaboration [121]. We have assumed five years of neutrino run and five years of anti-neutrino run to estimate the physics capabilities of this set-up.

In Europe, the proposed LBNO experiment [79-82] offers an interesting possibility to address the fundamental unsolved issues in neutrino oscillation physics using a baseline of $2290 \mathrm{~km}$ between CERN and Pyhäsalmi mine in Finland which enables us to cover a wide range of $L / E$ choices, mandatory to resolve parameter degeneracies. The Pyhäsalmi mine will house a giant $70 \mathrm{kt} \mathrm{LArTPC}$ as a far detector which will observe the neutrinos produced in a conventional wide-band beam facility at CERN. The fluxes that we use in our simulation have been computed assuming an exposure of $1.5 \times 10^{20}$ protons on target in 200 days per calendar year from the SPS accelerator at $400 \mathrm{GeV}$ with a beam power of $750 \mathrm{~kW}$ [122]. For LBNO also, we assume five years of neutrino run and five years of anti-neutrino run. We consider the same detector properties as that of DUNE.

\subsection{Event spectrum and rates}

In this section, we present the expected event spectra and total event rates for both the setups under consideration in the presence of long-range potential. We calculate the number of expected electron events ${ }^{10}$ in the $i$-th energy bin in the detector using the following expression

$$
N_{i}=\frac{T n_{n} \epsilon}{4 \pi L^{2}} \int_{0}^{E_{\max }} d E \int_{E_{A_{i}}^{\min }}^{E_{A_{i}}^{\max }} d E_{A} \phi(E) \sigma_{\nu_{e}}(E) R\left(E, E_{A}\right) P_{\mu e}(E),
$$

where $\phi(E)$ is the neutrino flux, $T$ is the total running time, $n_{n}$ is the number of target nucleons in the detector, $\epsilon$ is the detector efficiency, and $R\left(E, E_{A}\right)$ is the Gaußian energy resolution function of the detector. $\sigma_{\nu_{e}}$ is the neutrino interaction cross-section which has been taken from refs. [123, 124], where the authors estimated the cross-section for water and isoscalar targets. In order to have LAr cross-sections, we have scaled the inclusive charged current cross-sections of water by a factor of 1.06 for neutrino and 0.94 for antineutrino $[125,126]$. The quantities $E$ and $E_{A}$ are the true and reconstructed (anti-)neutrino energies respectively, and $L$ is the path length.

In our study, we consider the $\nu_{e}$ and $\bar{\nu}_{e}$ appearance channels, where the backgrounds mainly stem from the the intrinsic $\nu_{e} / \bar{\nu}_{e}$ contamination of the beam, the number of muon events which will be misidentified as electron events, and the neutral current events. In figure 7, we show the expected signal and background event spectra as a function of reconstructed neutrino energy including the efficiency and background rejection capabilities. The left panel shows the results for the DUNE set-up with $35 \mathrm{kt}$ far detector mass. The right panel displays the same for the LBNO set-up with $70 \mathrm{kt}$ far detector. In both the

\footnotetext{
${ }^{10}$ The number of positron events can be estimated using eq. (4.1), by considering appropriate oscillation probability and cross-section. The same is true for $\mu^{ \pm}$events.
} 

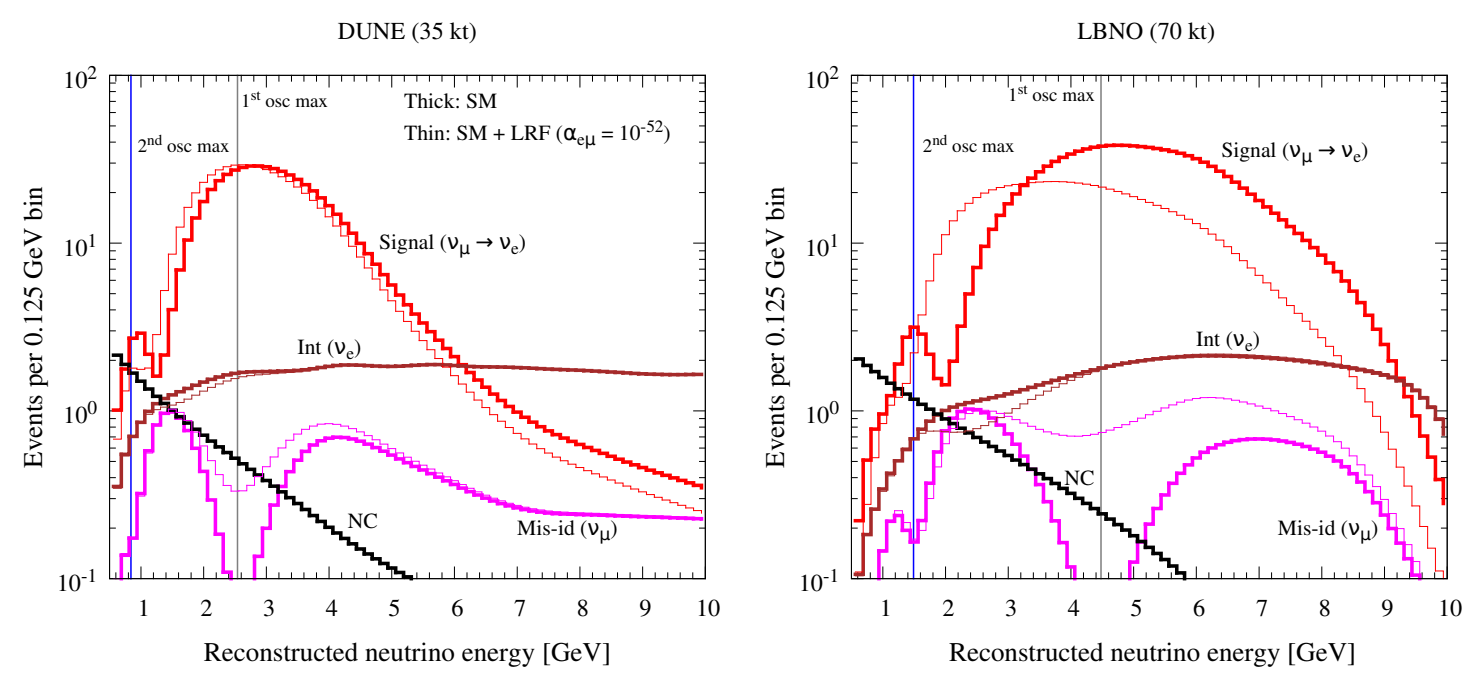

Figure 7. Expected signal and background event spectra in the $\nu_{e}$ appearance channel as a function of the reconstructed neutrino energy including the efficiency and background rejection capabilities. The left panel is for the DUNE (35 kt) and the right one is for the LBNO (70 kt). In each panel, the thick lines correspond to the SM case, whereas the thin lines are drawn assuming $\alpha_{e \mu}=10^{-52}$. In both the panels, the solid grey (blue) vertical lines display the locations of the first (second) oscillation maxima. We assume $\delta_{\mathrm{CP}}=0^{\circ}$ and $\mathrm{NH}$. For other oscillation parameters, the values are taken from the third column of table 2 .

panels, the thick lines correspond to the SM case, whereas the thin lines are drawn assuming $\alpha_{e \mu}=10^{-52}$. In both the set-ups, we can clearly see a systematic downward bias in the reconstructed energy for the neutral current background events due to the final state neutrino included via the migration matrices. The solid grey (blue) vertical lines display the locations of the first (second) oscillation maxima. The red solid histogram shows the signal event spectrum. Note that in the presence of long-range potential, both the signal and background (intrinsic $\nu_{e}$ contamination and misidentified muons) event spectra get modified substantially. For both the baselines, we have considerable number of signal events around the second oscillation maximum. But, these event samples are highly contaminated with the neutral current and other backgrounds at lower energies, limiting their impact.

In table 3, we show a comparison between the total signal and background event rates in the $\nu_{e} / \bar{\nu}_{e}$ appearance channel for DUNE (35 kt) and LBNO (70 kt) set-ups. For both the set-ups, we assume five years of neutrino run and five years of anti-neutrino run. For the cases denoted by 'SM+LRF', we take $\alpha_{e \mu}=10^{-52}$. The results are shown for both $\mathrm{NH}$ and $\mathrm{IH}$ assuming $\delta_{\mathrm{CP}}=0^{\circ}$. The Earth matter effects play an important role for both the baselines which is evident from the fact that in the neutrino channel, the number of expected events is quite large compared to the IH case and in the anti-neutrino channel, the situation is totally opposite where we have larger event rates for IH than for NH. The relative difference between the number of events for $\mathrm{NH}$ and $\mathrm{IH}$ is larger for the CERNPyhäsalmi baseline than the FNAL-Homestake baseline, since the impact of matter effects is more significant at the $2290 \mathrm{~km}$ baseline compared to the $1300 \mathrm{~km}$ baseline. In the 


\begin{tabular}{|c||c|c||c|c|}
\hline \multirow{2}{*}{ Channel } & \multicolumn{2}{|c||}{ DUNE (35 kt) } & \multicolumn{2}{c|}{ LBNO (70 kt) } \\
\cline { 2 - 5 } & Signal & Background & Signal & Background \\
\cline { 2 - 5 } & CC & Int+Mis-id+NC=Total & CC & Int+Mis-id+NC=Total \\
\hline \hline$P_{\mu e}(\mathrm{NH}, \mathrm{SM})$ & 590 & $125+29+24=178$ & 1228 & $115+31+29=175$ \\
\hline$P_{\mu e}(\mathrm{NH}, \mathrm{SM}+\mathrm{LRF})$ & 588 & $123+34+24=181$ & 786 & $112+53+29=194$ \\
\hline$P_{\mu e}(\mathrm{IH}, \mathrm{SM})$ & 268 & $129+29+24=182$ & 220 & $126+31+29=186$ \\
\hline$P_{\mu e}(\mathrm{IH}, \mathrm{SM}+\mathrm{LRF})$ & 108 & $130+33+24=187$ & 49 & $128+50+29=207$ \\
\hline \hline$P_{\bar{\mu} \bar{e}}(\mathrm{NH}, \mathrm{SM})$ & 116 & $43+10+7=60$ & 117 & $33+11+13=57$ \\
\hline$P_{\bar{\mu} \bar{e}(\mathrm{NH}, \mathrm{SM}+\mathrm{LRF})}$ & 44 & $44+12+7=63$ & 22 & $34+19+13=66$ \\
\hline$P_{\bar{\mu} \bar{e}}(\mathrm{IH}, \mathrm{SM})$ & 210 & $42+10+7=59$ & 484 & $30+11+13=54$ \\
\hline$P_{\bar{\mu} \bar{e}}(\mathrm{IH}, \mathrm{SM}+\mathrm{LRF})$ & 220 & $41+12+7=60$ & 343 & $29+19+13=61$ \\
\hline
\end{tabular}

Table 3. Comparison of the total signal and background event rates in the $\nu_{e} / \bar{\nu}_{e}$ appearance channel for DUNE (35 kt) and LBNO (70 kt) set-ups. Here 'Int' means intrinsic beam contamination, 'Mis-id' means misidentified muon events, and ' $\mathrm{NC}$ ' stands for neutral current. For the cases denoted by 'SM+LRF', we take $\alpha_{e \mu}=10^{-52}$. The results are shown for both $\mathrm{NH}$ and IH assuming $\delta_{\mathrm{CP}}$ $=0^{\circ}$. For both the set-ups, we assume five years of neutrino run and five years of anti-neutrino run.

presence of long-range potential with a benchmark choice of $\alpha_{e \mu}=10^{-52}$, qualitatively, the trend remains the same as mentioned above. Table 3 clearly shows that in all the cases, the most dominant contribution to the background comes from the intrinsic $\nu_{e} / \bar{\nu}_{e}$ beam contamination. Note that though the total signal event rate for the DUNE set-up in the neutrino mode with $\mathrm{NH}$, does not change much due to long-range potential with $\alpha_{e \mu}=10^{-52}$, but, the shape of the signal event spectrum gets affected by considerable amount as can be seen from figure 7, which enables us to place tight constraints on $\alpha_{e \mu}$ as we discuss in the results section. In our simulation, we also include the information coming from the $\nu_{\mu} / \bar{\nu}_{\mu}$ disappearance channels. For these type of channels, neutral current events are the main source of background. Table 4 shows the total signal and background event rates in the $\nu_{\mu} / \bar{\nu}_{\mu}$ disappearance channels for both the set-ups, considering five years of neutrino run and five years of anti-neutrino run. For the cases marked by 'SM+LRF', we take $\alpha_{e \mu}=10^{-52}$ and we present results for both $\mathrm{NH}$ and IH assuming $\delta_{\mathrm{CP}}=0^{\circ}$. Interestingly, the $\nu_{\mu} / \bar{\nu}_{\mu}$ disappearance channels are also quite sensitive to the long-range potential and in all the cases, we see a significant change in the total signal event rates with $\alpha_{e \mu}=10^{-52}$ as compared to the SM case. Also, the rates are different for $\mathrm{NH}$ and IH in the presence of long-range potential. The $\nu_{\mu} / \bar{\nu}_{\mu}$ disappearance channels also play an important role to constrain the atmospheric oscillation parameters in the fit.

\subsection{Bi-events plot}

In this section, we make an attempt to unravel the impact of long-range potential with the help of bi-events plot. In figure 8 , we have plotted $\nu_{e}$ vs. $\bar{\nu}_{e}$ appearance events, for DUNE (left panel) and LBNO (right panel), considering both $\mathrm{NH}$ and $\mathrm{IH}$ and with and without long-range potential. Since $\delta_{\mathrm{CP}}$ is not known, events are generated for the full 


\begin{tabular}{|c||c|c||c|c|}
\hline \multirow{2}{*}{ Channel } & \multicolumn{2}{c||}{ DUNE (35 kt) } & \multicolumn{2}{c|}{ LBNO (70 kt) } \\
\cline { 2 - 5 } & Signal & Background & Signal & Background \\
\cline { 2 - 5 } & CC & NC & CC & NC \\
\hline \hline$P_{\mu \mu}(\mathrm{NH}, \mathrm{SM})$ & 4889 & 24 & 5222 & 29 \\
\hline$P_{\mu \mu}(\mathrm{NH}, \mathrm{SM}+\mathrm{LRF})$ & 5806 & 24 & 8949 & 29 \\
\hline$P_{\mu \mu}(\mathrm{IH}, \mathrm{SM})$ & 4882 & 24 & 5203 & 29 \\
\hline$P_{\mu \mu}(\mathrm{IH}, \mathrm{SM}+\mathrm{LRF})$ & 5569 & 24 & 8519 & 29 \\
\hline \hline$P_{\bar{\mu} \bar{\mu}}(\mathrm{NH}, \mathrm{SM})$ & 1751 & 7 & 1936 & 13 \\
\hline$P_{\bar{\mu} \bar{\mu}}(\mathrm{NH}, \mathrm{SM}+\mathrm{LRF})$ & 2012 & 7 & 3257 & 13 \\
\hline$P_{\bar{\mu} \bar{\mu}}(\mathrm{IH}, \mathrm{SM})$ & 1752 & 7 & 1923 & 13 \\
\hline$P_{\bar{\mu} \bar{\mu}}(\mathrm{IH}, \mathrm{SM}+\mathrm{LRF})$ & 2063 & 7 & 3309 & 13 \\
\hline \multicolumn{2}{||c|}{}
\end{tabular}

Table 4. Comparison of the total signal and background event rates in the $\nu_{\mu} / \bar{\nu}_{\mu}$ disappearance channel for DUNE (35 kt) and LBNO (70 kt) set-ups. For the cases denoted by 'SM+LRF', we take $\alpha_{e \mu}=10^{-52}$. The results are shown for both $\mathrm{NH}$ and $\mathrm{IH}$ assuming $\delta_{\mathrm{CP}}=0^{\circ}$. For both the set-ups, we consider five years of neutrino run and five years of anti-neutrino run.

range $\left[-180^{\circ}, 180^{\circ}\right]$, leading to the ellipses. For the cases labelled by 'SM+LRF', we take $\alpha_{e \mu}=10^{-52}$. We generate these plots with $\sin ^{2} \theta_{23}=0.5$ as mentioned in table 2 . The ellipses in figure 8 suggest that both the set-ups can discriminate between $\mathrm{NH}$ and $\mathrm{IH}$ at high confidence level, irrespective of the choice of $\delta_{\mathrm{CP}}$, and the presence of long-range potential with $\alpha_{e \mu}=10^{-52}$ does not spoil this picture. We can see from the left panel that for the DUNE set-up, the anti-neutrino (neutrino) event rates get reduced for NH (IH) with LRF as compared to the SM case. But, for CERN-Pyhäsalmi baseline (see right panel) with more matter effect, both the neutrino and anti-neutrino event rates get diminished for $\mathrm{NH}$ and IH in the presence of long-range potential as compared to the SM case. Now, let us make an attempt to understand this behavior. In the presence of long-range potential, the locations of the first oscillation maxima shift towards lower energies (see the upper panels of figure 5 and figure 16), where both the fluxes and the interaction cross-sections are small. On the contrary, at higher energies, we see a suppression in the probability where we have most of the neutrino fluxes and the cross-sections are also high at these energies. These opposite bahaviors are responsible for the large depletions in the event rates. Figure 8 also portrays that the asymmetries between the neutrino and anti-neutrino appearance events are largest for the combinations: $\left(\mathrm{NH}, \delta_{\mathrm{CP}}=-90^{\circ}\right)$ and $\left(\mathrm{IH}, \delta_{\mathrm{CP}}=90^{\circ}\right)$. One striking feature emerging from both the panels is that all the ellipses get shrunk in the presence of long-range potential, reducing the differences in the number of events due to the $\mathrm{CP}$-conserving and $\mathrm{CP}$-violating phases. It ultimately affects the $\mathrm{CP}$-coverage for the leptonic $\mathrm{CP}$-violation searches, where we study the choices of the $\mathrm{CP}$ phase, $\delta_{\mathrm{CP}}$ which can be distinguished from both $0^{\circ}$ and $180^{\circ}$ at a given confidence level. The right panel shows that this effect is more prominent for the LBNO set-up, severely limiting its discovery reach for CP-violation which we discuss in detail in the results section. 

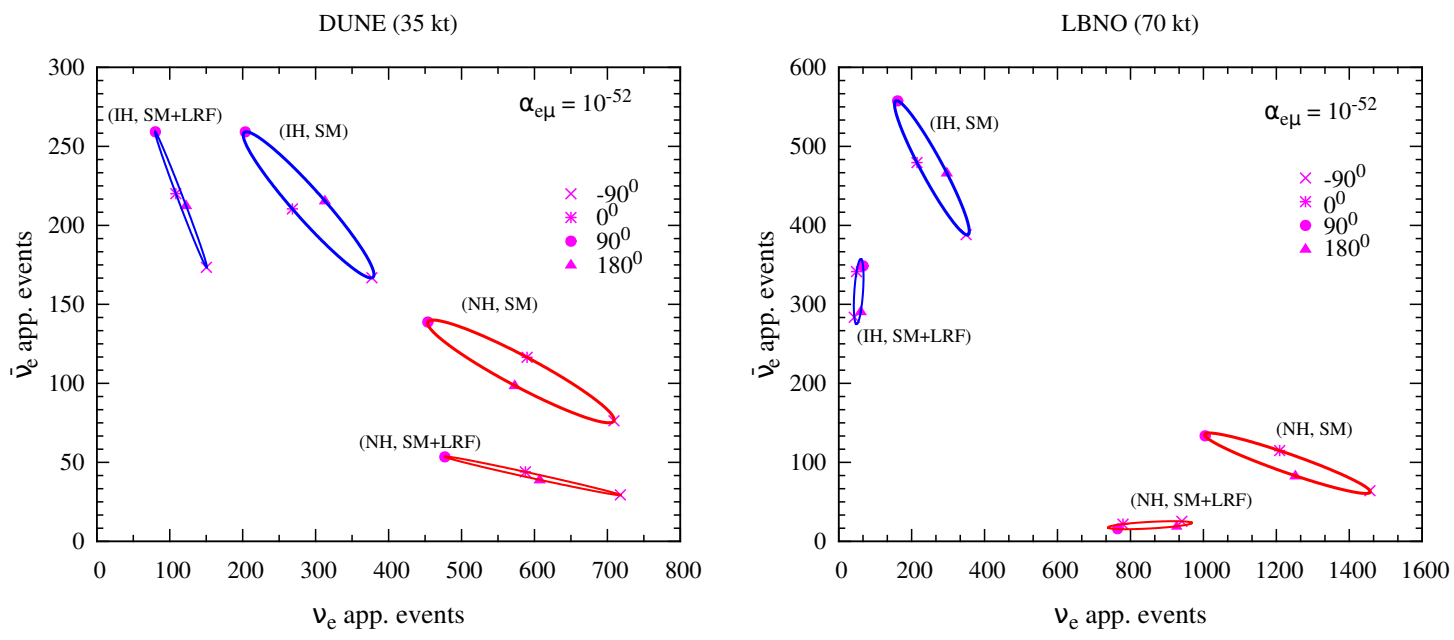

Figure 8. Bi-events $\left(\nu_{e}\right.$ and $\bar{\nu}_{e}$ appearance) plots for $\mathrm{NH}$ and $\mathrm{IH}$ with and without long-range potential. The ellipses are due to all possible $\delta_{\mathrm{CP}}$ values. For the cases labelled by 'SM+LRF', we take $\alpha_{e \mu}=10^{-52}$. The left panel is for the DUNE set-up (35 kt) and the right panel is for the LBNO set-up (70 kt).

\section{Simulation method}

In this section, we give a brief description of the numerical technique and analysis procedure which we adopt to estimate the physics reach of the experimental set-ups. We have made suitable changes in the GLoBES software $[127,128]$ to obtain our results. The entire numerical analysis is performed using the full three-flavor oscillation probabilities. Unless stated otherwise, we generate our simulated data considering the true values of the oscillation parameters given in the third column of table 2. These choices of the oscillation parameters are well within their $1 \sigma$ allowed ranges which are obtained in recent global fit analysis [52]. In the fit, we marginalize over test $\sin ^{2} \theta_{23}$ and test $\delta_{\mathrm{CP}}$ in their allowed ranges which are given in the fourth column of table 2 , without assuming any prior on these parameters. We also marginalize over both the hierarchy choices in the fit for all the analyses, except for the mass hierarchy discovery studies where our goal is to exclude the wrong hierarchy in the fit. We keep $\theta_{13}$ fixed in the fit as the Daya Bay experiment is expected to achieve a relative $1 \sigma$ precision of $\sim 3 \%$ by the end of 2017 [129], and needless to say that the global oscillation data will severely constrain $\theta_{13}$ beyond the Daya Bay limit before these future experiments will come online. For the atmospheric mass-squared splitting, we take the true value of $\Delta m_{\mu \mu}^{2}= \pm 2.4 \times 10^{-3} \mathrm{eV}^{2}$ where positive (negative) sign is for $\mathrm{NH}(\mathrm{IH})$, and we do not marginalize over this parameter in the fit since the projected combined data from the currently running $\mathrm{T} 2 \mathrm{~K}$ and $\mathrm{NO} \nu \mathrm{A}$ experiments will be able to improve the precision in $\left|\Delta m_{\mu \mu}^{2}\right|$ to sub-percent level for maximal $\theta_{23}$ [130]. On top of the standard three-flavor oscillation parameters, we have also the LRF parameter $\alpha_{e \mu}$ which enters into the oscillation probability. As far as the true and test values of $\alpha_{e \mu}$ are 
concerned, we vary our choices in the range $10^{-54}$ to $10^{-51}$, where the lower limit ${ }^{11}$ corresponds to the cases where the oscillation probabilities almost overlap with the SM cases for the set-ups that we consider in this work. We take the upper limit of $\alpha_{e \mu}$ as $10^{-51}$ which covers all the existing bounds on this parameter available from the oscillation experiments as discussed in section 2.2. Based on the techniques discussed in refs. [131, 132], we use the following $\chi^{2}$ function in our statistical analysis:

$$
\chi^{2}=\min _{\xi_{s}, \xi_{b}}\left[2 \sum_{i=1}^{n}\left(\tilde{y}_{i}-x_{i}-x_{i} \ln \frac{\tilde{y}_{i}}{x_{i}}\right)+\xi_{s}^{2}+\xi_{b}^{2}\right],
$$

where $n$ is the total number of bins and

$$
\tilde{y}_{i}\left(\left\{\omega, \alpha_{e \mu}\right\},\left\{\xi_{s}, \xi_{b}\right\}\right)=N_{i}^{t h}\left(\left\{\omega, \alpha_{e \mu}\right\}\right)\left[1+\pi^{s} \xi_{s}\right]+N_{i}^{b}\left(\left\{\omega, \alpha_{e \mu}\right\}\right)\left[1+\pi^{b} \xi_{b}\right] .
$$

Above, $N_{i}^{t h}\left(\left\{\omega, \alpha_{e \mu}\right\}\right)$ is the predicted number of signal events in the $i$-th energy bin for a set of oscillation parameters $\omega$ and a particular value of $\alpha_{e \mu} . N_{i}^{b}\left(\left\{\omega, \alpha_{e \mu}\right\}\right)$ is the number of background events in the $i$-th bin where the charged current backgrounds are dependent on $\omega$ and $\alpha_{e \mu}$, and the neutral current backgrounds do not depend on the oscillation parameters and $\alpha_{e \mu}$. The quantities $\pi^{s}$ and $\pi^{b}$ in eq. (5.2) are the systematic errors on the signal and background respectively. For both the set-ups, we consider $\pi^{s}=5 \%$ and $\pi^{b}=5 \%$ in the form of normalization error for both the appearance and disappearance channels. The quantities $\xi_{s}$ and $\xi_{b}$ are the "pulls" due to the systematic error on signal and background respectively. We incorporate the data in eq. (5.1) through the variable $x_{i}=N_{i}^{e x}+N_{i}^{b}$, where $N_{i}^{e x}$ is the number of observed charged current signal events in the $i$-th energy bin and $N_{i}^{b}$ is the background as mentioned earlier. To estimate the total $\chi^{2}$, we add the $\chi^{2}$ contributions coming from all the relevant channels in a given experiment in the following way

$$
\chi_{\text {total }}^{2}=\chi_{\nu_{\mu} \rightarrow \nu_{e}}^{2}+\chi_{\nu_{\mu} \rightarrow \nu_{\mu}}^{2}+\chi_{\bar{\nu}_{\mu} \rightarrow \bar{\nu}_{e}}^{2}+\chi_{\bar{\nu}_{\mu} \rightarrow \bar{\nu}_{\mu}}^{2},
$$

where we assume that all these channels are completely uncorrelated, all the energy bins in a given channel are fully correlated, and the systematic errors on signal and background are fully uncorrelated. Finally, $\chi_{\text {total }}^{2}$ is marginalized in the fit over the oscillation parameters, both the hierarchy choices, the LRF parameter $\alpha_{e \mu}$ (as needed), and the systematic parameters as mentioned above to obtain $\Delta \chi_{\min }^{2}$.

\section{Results}

In this section, we report our main findings. First, we present the expected constraints on $\alpha_{e \mu}$ from the proposed DUNE and LBNO experiments. Next, we quantify the discovery reach for $\alpha_{e \mu}$ of these future facilities. Then, we address how the flavor-dependent LRF, mediated by the extremely light $L_{e}-L_{\mu}$ gauge boson can affect the CP-violation searches and the mass hierarchy measurements at these upcoming facilities.

\footnotetext{
${ }^{11}$ If the range of the long-range force is equal or larger than our distance from the galactic center, then the collective long-range potential due to all the electrons in the galaxy becomes significant. In such cases, these experimental set-ups can be sensitive to even lower values of $\alpha_{e \mu}$ [88].
} 

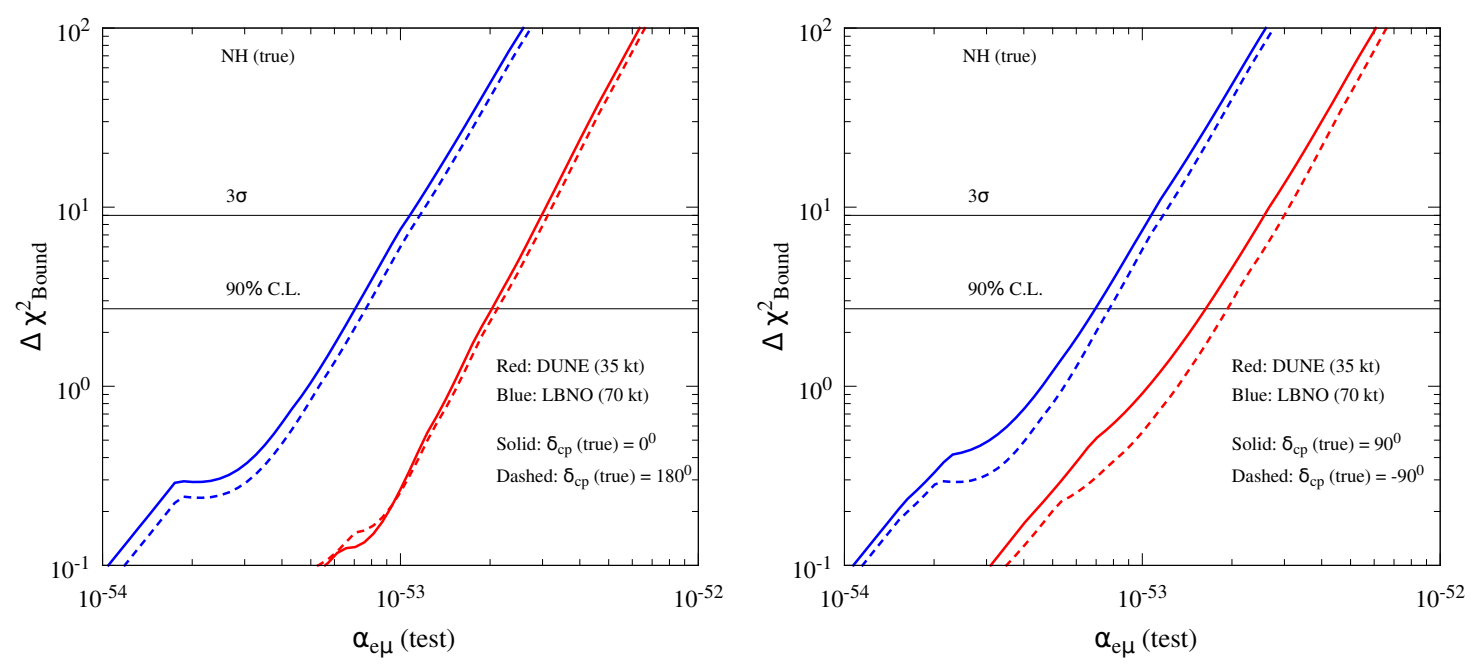

Figure 9. Expected bounds on $\alpha_{e \mu}$ from the DUNE (35 kt) and LBNO (70 kt) experiments in the scenarios when the data show no signal of LRF. Results are given for four different choices of true values of $\delta_{\mathrm{CP}}$. The left panel is for CP-conserving choices: $\delta_{\mathrm{CP}}($ true $)=0^{\circ}$ (solid lines), $180^{\circ}$ (dashed lines). The right panel is for maximal CP-violating choices: $\delta_{\mathrm{CP}}$ (true) $=90^{\circ}$ (solid lines), $-90^{\circ}$ (dashed lines). In all the cases, we assume NH as true hierarchy.

\subsection{Expected constraints on the effective gauge coupling $\alpha_{e \mu}$}

In this section, we estimate the upper bounds on $\alpha_{e \mu}$ from the proposed DUNE and LBNO experiments if there is no signal of LRF in the data. This performance indicator corresponds to the new upper limit on $\alpha_{e \mu}$ if the experiment does not see a signal of LRF in oscillations. We simulate this situation in our analysis by generating the data at $\alpha_{e \mu}$ (true) $=0$ and fitting it with some non-zero value of $\alpha_{e \mu}$ by means of the $\chi^{2}$ technique as outlined in section 5 . The corresponding $\Delta \chi_{\text {Bound }}^{2}$ obtained after marginalizing over oscillation parameters $\left(\theta_{23}\right.$, $\delta_{\mathrm{CP}}$, and mass hierarchy) and systematic parameters in the fit, is plotted in figure 9 as a function of $\alpha_{e \mu}$ (test), which gives a measure of the sensitivity reach of the DUNE or LBNO set-up to the effective gauge coupling of the LRF. New limits are given for four different choices of true values of $\delta_{\mathrm{CP}}$. The left panel is for CP-conserving choices: $\delta_{\mathrm{CP}}($ true $)=$ $0^{\circ}$ (solid lines), $180^{\circ}$ (dashed lines). The right panel is for maximal CP-violating choices: $\delta_{\mathrm{CP}}($ true $)=90^{\circ}$ (solid lines), $-90^{\circ}$ (dashed lines). In all the cases, we assume NH as true hierarchy. Figure 9 clearly shows that the LBNO set-up with 70 kt detector mass can place better limits on $\alpha_{e \mu}$ as compared to the DUNE set-up with $35 \mathrm{kt}$ detector and the limits are not very sensitive to the choice of unknown $\delta_{\mathrm{CP}}($ true) for both the set-ups. Table 5 lists the precise upper limits on $\alpha_{e \mu}$ which are expected from these future facilities if there is no trace of LRF in the data. We present the bounds at $90 \%(1.64 \sigma)$ and $3 \sigma$ confidence levels $^{12}$ for four different choices of true values of $\delta_{\mathrm{CP}}: 0^{\circ}, 180^{\circ}, 90^{\circ}$, and $-90^{\circ}$. For each $\delta_{\mathrm{CP}}($ true $)$ value, we give the results for both $\mathrm{NH}$ and $\mathrm{IH}$ as true hierarchy choice. For an example,

\footnotetext{
${ }^{12}$ To calculate this, we use the relation $\mathrm{n} \sigma=\sqrt{\Delta \chi_{\min }^{2}}$. In [133], it was shown that the above relation is valid in the frequentist method of hypothesis testing.
} 


\begin{tabular}{|c|c|c|c|c|c|c|c|}
\hline Expt. & $\delta_{\mathrm{CP}}($ true $)$ & 90\% C.L. & $3 \sigma$ & Expt. & $\delta_{\mathrm{CP}}($ true $)$ & 90\% C.L. & $3 \sigma$ \\
\hline \multirow{8}{*}{$\begin{array}{l}\text { DUNE } \\
(35 \mathrm{kt})\end{array}$} & $\Omega^{0}$ & $2.0 \times 10^{-53}(\mathrm{NH})$ & $3.0 \times 10^{-53}(\mathrm{NH})$ & \multirow{8}{*}{$\begin{array}{l}\text { LBNO } \\
(70 \mathrm{kt})\end{array}$} & \multirow{2}{*}{$0^{0}$} & $7.0 \times 10^{-54}(\mathrm{NH})$ & $1.1 \times 10^{-53}(\mathrm{NH})$ \\
\hline & 0 & $2.1 \times 10^{-53}(\mathrm{IH})$ & $3.1 \times 10^{-53}(\mathrm{NH})$ & & & $7.8 \times 10^{-54}(\mathrm{IH})$ & $1.2 \times 10^{-53}(\mathrm{IH})$ \\
\hline & \multirow{2}{*}{$180^{\circ}$} & $2.1 \times 10^{-53}(\mathrm{NH})$ & $3.1 \times 10^{-53}(\mathrm{NH})$ & & \multirow{2}{*}{$180^{\circ}$} & $7.6 \times 10^{-54}(\mathrm{NH})$ & $1.2 \times 10^{-53}(\mathrm{NH})$ \\
\hline & & $2.0 \times 10^{-53}(\mathrm{IH})$ & $3.0 \times 10^{-53}(\mathrm{IH})$ & & & $7.0 \times 10^{-54}(\mathrm{IH})$ & $1.1 \times 10^{-53}(\mathrm{IH})$ \\
\hline & \multirow{2}{*}{$90^{\circ}$} & $1.7 \times 10^{-53}(\mathrm{NH})$ & $2.7 \times 10^{-53}(\mathrm{NH})$ & & \multirow{2}{*}{$90^{\circ}$} & $7.0 \times 10^{-54}(\mathrm{NH})$ & $1.1 \times 10^{-53}(\mathrm{NH})$ \\
\hline & & $1.8 \times 10^{-53}(\mathrm{IH})$ & $2.8 \times 10^{-53}(\mathrm{IH})$ & & & $7.0 \times 10^{-54}(\mathrm{IH})$ & $1.1 \times 10^{-53}(\mathrm{IH})$ \\
\hline & \multirow{2}{*}{$-90^{0}$} & $1.9 \times 10^{-53}(\mathrm{NH})$ & $3.0 \times 10^{-53}(\mathrm{NH})$ & & \multirow{2}{*}{$-90^{0}$} & $7.8 \times 10^{-54}(\mathrm{NH})$ & $1.2 \times 10^{-53}(\mathrm{NH})$ \\
\hline & & $1.5 \times 10^{-53}(\mathrm{IH})$ & $2.4 \times 10^{-53}(\mathrm{IH})$ & & & $6.0 \times 10^{-54}(\mathrm{IH})$ & $9.6 \times 10^{-54}(\mathrm{IH})$ \\
\hline
\end{tabular}

Table 5. The expected bounds on $\alpha_{e \mu}$ from the DUNE (35 kt) and LBNO (70 kt) experiments if there is no signal of LRF in the data. The results are presented at $90 \%$ and $3 \sigma$ confidence levels for four different choices of true values of $\delta_{\mathrm{CP}}: 0^{\circ}, 180^{\circ}, 90^{\circ}$, and $-90^{\circ}$. For each $\delta_{\mathrm{CP}}($ true) value, we show the results for both the choices of true hierarchy: $\mathrm{NH}$ and $\mathrm{IH}$.

if $\delta_{\mathrm{CP}}$ (true) $=-90^{\circ}$ and true hierarchy is $\mathrm{NH}$, then the $90 \%$ C.L. limit from the DUNE (LBNO) experiment is $\alpha_{e \mu}<1.9 \times 10^{-53}\left(7.8 \times 10^{-54}\right)$, suggesting that the constraint from the LBNO experiment is $\sim 2.4$ times better than the DUNE set-up. ${ }^{13}$ This future limit from the DUNE (LBNO) experiment is $\sim 30$ (70) times better than the existing $\operatorname{limit}^{14}$ from the SK experiment [85] which is also mainly sensitive to the atmospheric mass scale like long-baseline experiments. At $3 \sigma$, we see the same relative improvement in the LBNO experiment in constraining $\alpha_{e \mu}$ compared to the DUNE set-up. Table 5 also suggests that the limits are not highly dependent on the true choices of mass hierarchy. The variation in the upper limits on $\alpha_{e \mu}$ is also not significant as we vary $\delta_{\mathrm{CP}}$ (true) in the range $-180^{\circ}$ to $180^{\circ}$ as can be seen from figure 10 , where we give the expected bounds at $3 \sigma$ and $5 \sigma$ confidence levels from both the set-ups assuming $\mathrm{NH}$ as true hierarchy. Next, we discuss the discovery reach for $\alpha_{e \mu}$ if we find a positive signal of LRF in the expected event spectra at DUNE and LBNO.

\subsection{Discovery reach for $\alpha_{e \mu}$}

How good are our chances of observing a positive signal for LRF and hence $\alpha_{e \mu}$ in these proposed facilities? We answer this question in terms of the parameter indicator which we call the "discovery reach" of the experiment for $\alpha_{e \mu}$. We define this performance indicator as the expected lower limit on true values of $\alpha_{e \mu}$ above which the projected data at DUNE or LBNO would give us a signal for LRF at a certain confidence level. To find these limiting values, we simulate the data for various true values of $\alpha_{e \mu}$ and fit it with a predicted event spectrum corresponding to $\alpha_{e \mu}=0$. We marginalize over $\theta_{23}, \delta_{\mathrm{CP}}$, mass hierarchy, and systematic parameters in the fit to estimate the resultant $\Delta \chi_{\text {Discovery }}^{2}$ which is plotted in

\footnotetext{
${ }^{13}$ We have checked that the larger detector mass (two times) in the LBNO set-up compared to the DUNE set-up is partially responsible for this improvement in the sensitivity, but also the larger path length with more matter effect plays an important role in this direction.

${ }^{14}$ This limit is quite old and was derived in a two-flavor scheme assuming $\theta_{13}=0^{\circ}$ [85]. One needs to revise this limit using the presently available full data set from the SK experiment in light of the non-zero and large $\theta_{13}$.
} 


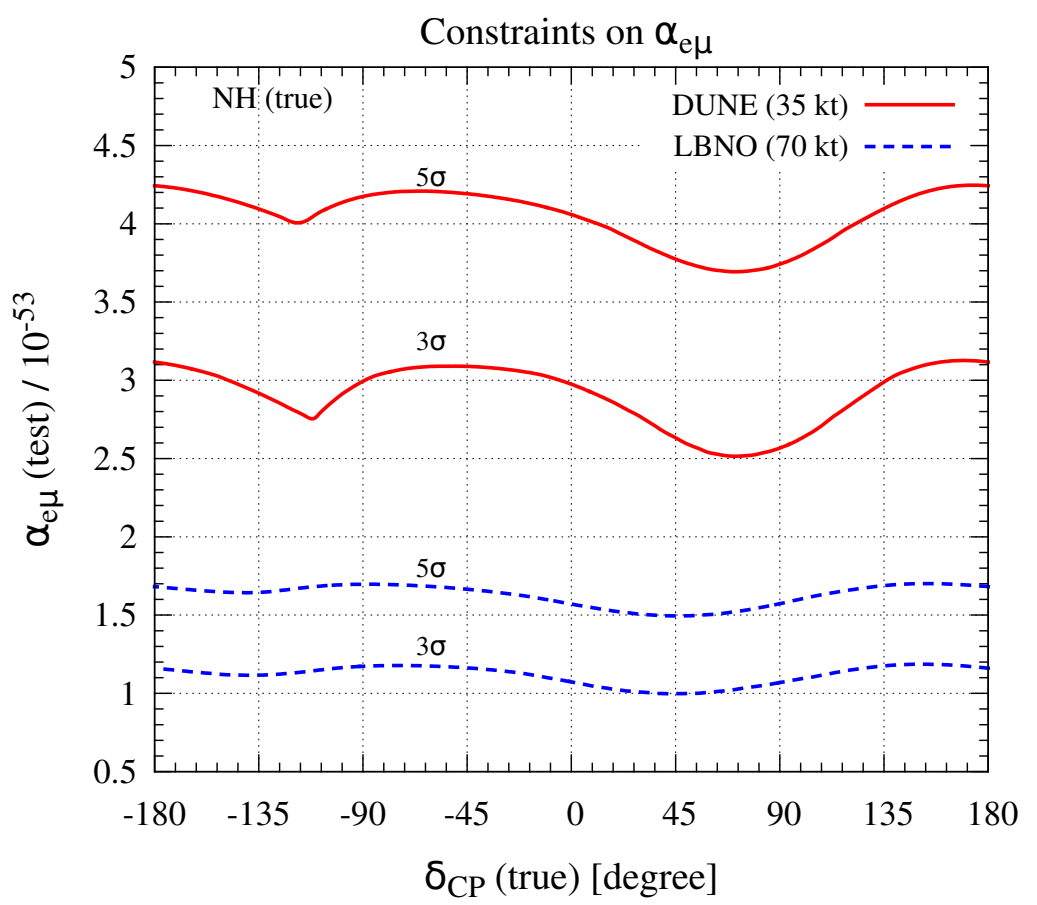

Figure 10. Constraints on $\alpha_{e \mu}$ as a function of true value of $\delta_{\mathrm{CP}}$ assuming $\mathrm{NH}$ as true hierarchy. Results are shown for DUNE (35 kt) and LBNO (70 kt) at $3 \sigma$ and $5 \sigma$ confidence levels.

figure 11 for DUNE (35 kt) and LBNO (70 kt) set-ups, considering four different choices of true values of $\delta_{\mathrm{CP}}$. In the left panel, we take the CP-conserving choices: $\delta_{\mathrm{CP}}($ true $)=0^{\circ}$ (solid lines), $180^{\circ}$ (dashed lines). In the right panel, we consider the maximal CP-violating choices: $\delta_{\mathrm{CP}}($ true $)=90^{\circ}$ (solid lines), $-90^{\circ}$ (dashed lines). In all the cases, we take $\mathrm{NH}$ as true hierarchy. The nature of the curves in figure 11 are quite similar to the curves which are shown in figure 9, and LBNO with $70 \mathrm{kt}$ detector has better discovery reach for $\alpha_{e \mu}$ as compared to DUNE with $35 \mathrm{kt}$, like in the case of constraints on $\alpha_{e \mu}$. In table 6 , we give the precise lower limits on true values of $\alpha_{e \mu}$ which can be separated from $\alpha_{e \mu}=0$ in the fit at $90 \%$ and $3 \sigma$ confidence levels. The results are given for both the set-ups and for four different choices of true values of $\delta_{\mathrm{CP}}: 0^{\circ}, 180^{\circ}, 90^{\circ}$, and $-90^{\circ}$. For each $\delta_{\mathrm{CP}}($ true) value, we show the results for both the choices of true hierarchy: $\mathrm{NH}$ and IH. If we compare the entries in table 6 and table 5, then we can see that the values of the discovery reach for $\alpha_{e \mu}$ are slightly different than the constraints on $\alpha_{e \mu}$ at a given confidence level and for the same choices of true oscillation parameters. Also, we can see that the values of discovery reach are marginally dependent on the choices of true $\delta_{\mathrm{CP}}$ and mass hierarchy as we have seen for the constraints in the previous section.

\subsection{How robust are CP-violation searches in presence of LRF?}

This section is devoted to study how the long-range potential due to $L_{e}-L_{\mu}$ symmetry affects the CP-violation search which is the prime goal of these future facilities. Can we reject both the $\mathrm{CP}$-conserving values of $0^{\circ}, 180^{\circ}$ at a given confidence level? The 

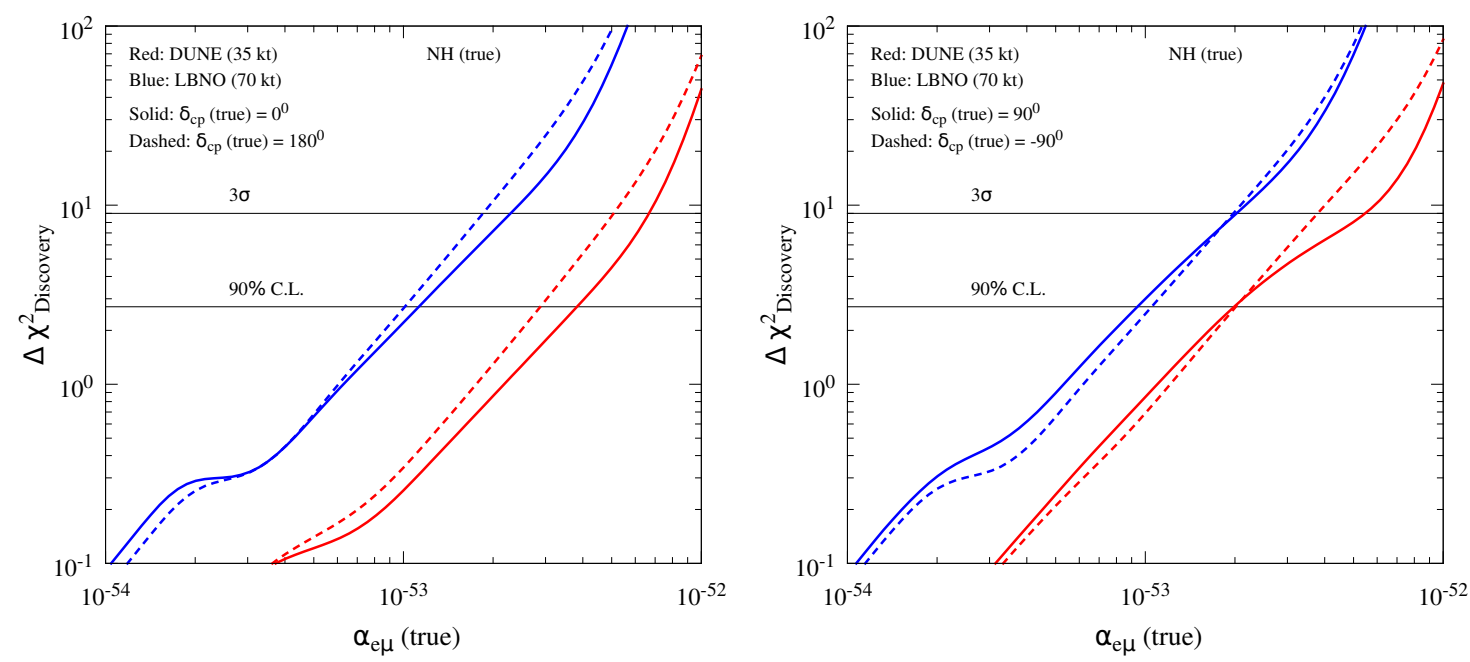

Figure 11. $\Delta \chi_{\text {Discovery }}^{2}$ showing the discovery reach for $\alpha_{e \mu}$ expected from the DUNE (35 kt) and LBNO (70 kt) set-ups in the cases when one finds a signal of LRF in the data. Results are given for four different choices of true values of $\delta_{\mathrm{CP}}$. The left panel is for CP-conserving choices: $\delta_{\mathrm{CP}}($ true $)=$ $0^{\circ}$ (solid lines), $180^{\circ}$ (dashed lines). The right panel is for maximal CP-violating choices: $\delta_{\mathrm{CP}}($ true) $=90^{\circ}$ (solid lines), $-90^{\circ}$ (dashed lines). In all the cases, we assume $\mathrm{NH}$ as true hierarchy.

\begin{tabular}{|c|c|c|c|c|c|c|c|}
\hline Expt. & $\delta_{\mathrm{CP}}($ true $)$ & 90\% C.L. & $3 \sigma$ & cpt. & $\delta_{\mathrm{CP}}$ (true) & 90\% C.L. & $3 \sigma$ \\
\hline \multirow{8}{*}{$\begin{array}{l}\text { DUNE } \\
(35 \mathrm{kt})\end{array}$} & & $3.8 \times 10^{-53}(\mathrm{NH})$ & $6.5 \times 10^{-53}(\mathrm{NH})$ & \multirow{8}{*}{$\begin{array}{l}\text { LBNO } \\
(70 \mathrm{kt})\end{array}$} & \multirow{2}{*}{$0^{0}$} & $1.2 \times 10^{-53}(\mathrm{NH})$ & $2.2 \times 10^{-53}(\mathrm{NH})$ \\
\hline & & $3.7 \times 10^{-53}(\mathrm{IH})$ & $7.2 \times 10^{-53}(\mathrm{IH})$ & & & $1.4 \times 10^{-53}(\mathrm{IH})$ & $2.6 \times 10^{-53}(\mathrm{IH})$ \\
\hline & \multirow{2}{*}{$180^{\circ}$} & $2.9 \times 10^{-53}(\mathrm{NH})$ & $5.0 \times 10^{-53}(\mathrm{NH})$ & & \multirow{2}{*}{$180^{\circ}$} & $9.5 \times 10^{-54}(\mathrm{NH})$ & $1.9 \times 10^{-53}(\mathrm{NH})$ \\
\hline & & $4.0 \times 10^{-53}(\mathrm{IH})$ & $7.8 \times 10^{-53}(\mathrm{IH})$ & & & $1.4 \times 10^{-53}(\mathrm{IH})$ & $2.6 \times 10^{-53}(\mathrm{IH})$ \\
\hline & \multirow{2}{*}{$90^{\circ}$} & $2.0 \times 10^{-53}(\mathrm{NH})$ & $5.4 \times 10^{-53}(\mathrm{NH})$ & & \multirow{2}{*}{$90^{\circ}$} & $9.0 \times 10^{-54}(\mathrm{NH})$ & $2.0 \times 10^{-53}(\mathrm{NH})$ \\
\hline & & $2.3 \times 10^{-53}(\mathrm{IH})$ & $4.4 \times 10^{-53}(\mathrm{IH})$ & & & $1.0 \times 10^{-53}(\mathrm{IH})$ & $2.0 \times 10^{-53}(\mathrm{IH})$ \\
\hline & \multirow[t]{2}{*}{$-90^{0}$} & $2.0 \times 10^{-53}(\mathrm{NH})$ & $3.7 \times 10^{-53}(\mathrm{NH})$ & & \multirow[t]{2}{*}{$-90^{0}$} & $1.0 \times 10^{-53}(\mathrm{NH})$ & $2.0 \times 10^{-53}(\mathrm{NH})$ \\
\hline & & $1.8 \times 10^{-53}(\mathrm{IH})$ & $4.8 \times 10^{-53}(\mathrm{IH})$ & & & $7.6 \times 10^{-54}(\mathrm{IH})$ & $1.5 \times 10^{-53}(\mathrm{IH})$ \\
\hline
\end{tabular}

Table 6. The discovery reach for $\alpha_{e \mu}$ as expected from the DUNE (35 kt) and LBNO (70 kt) experiments if the data show a signal of LRF. The results are presented at $90 \%$ and $3 \sigma$ confidence levels for four different choices of true values of $\delta_{\mathrm{CP}}: 0^{\circ}, 180^{\circ}, 90^{\circ}$, and $-90^{\circ}$. For each $\delta_{\mathrm{CP}}($ true) value, we show the results for both the choices of true hierarchy: $\mathrm{NH}$ and $\mathrm{IH}$.

performance indicator "discovery reach of leptonic CP-violation" addresses this question and obviously, this measurement becomes extremely difficult for the $\delta_{\mathrm{CP}}$ values which are close to $0^{\circ}$ and $180^{\circ}$. In figure 12 , we present the CP-violation discovery reach of DUNE (left panel) and LBNO (right panel) as a function of true value of $\delta_{\mathrm{CP}}$ assuming $\mathrm{NH}$ as true hierarchy. In this plot, we generate our predicted event spectrum (data) considering the true value of $\delta_{\mathrm{CP}}$ as shown in the x-axis, along with the other true values of the oscillation parameters given in the third column of table 2 . Then, we estimate the various theoretical event spectra assuming the test $\delta_{\mathrm{CP}}$ to be the CP-conserving values $0^{\circ}$ and $180^{\circ}$, and by 
varying simultaneously $\theta_{23}$ in its $3 \sigma$ allowed range and both the choices of mass hierarchy. We calculate the $\Delta \chi^{2}$ between each set of predicted and theoretical event spectra using the procedure described in section 5 . The smallest of all such $\Delta \chi^{2}$ values: $\Delta \chi_{\mathrm{CPV}}^{2}$ is plotted in figure 12 as a function of $\delta_{\mathrm{CP}}$ (true) in the range $-180^{\circ}$ to $180^{\circ}$. In both the panels, the solid red lines depict the 'SM' case where $\alpha_{e \mu}=0$ in the data and also in the fit. For each $\delta_{\mathrm{CP}}$ (true), we also give the results generating the data with three different true values of $\alpha_{e \mu}$ which are mentioned in the figure legends. In all these three cases, in the fit, we also marginalize over test values of $\alpha_{e \mu}$ in the range $10^{-54}$ to $10^{-51}$ along with the other three-flavor oscillation parameters as discussed before. Figure 12 clearly shows that the $\mathrm{CP}$-violation discovery reach can be altered by substantial amount as compared to the 'SM' case depending on the true choice of $\alpha_{e \mu}$. In case of $\alpha_{e \mu}$ (true) $=6 \times 10^{-53}$, we see a large suppression in the CP-violation discovery reach of DUNE (left panel) in the range $45^{\circ} \leq \delta_{\mathrm{CP}}$ (true) $\leq 135^{\circ}$. We have checked that this mainly happens due to the marginalization over $\theta_{23}$ in the fit where we vary $\sin ^{2} \theta_{23}$ over a wide range (0.38 to 0.64 ) without imposing any prior on it. In case $\alpha_{e \mu}$ (true) $=10^{-52}$, the LBNO set-up (right panel) suffers a large depletion in the $\mathrm{CP}$-violation discovery reach which can be easily explained with the help of bi-events plot (figure 8) shown in section 4.3. In the right panel of figure 8 , we have seen a large reduction in the $\nu$ and $\bar{\nu}$ event rates for CERN-Pyhäsalmi baseline with $\alpha_{e \mu}=10^{-52}$ and this is true for both $\mathrm{NH}$ and IH. The differences in the number of events for the $\mathrm{CP}$-conserving and $\mathrm{CP}$-violating phases get reduced as the ellipses in figure 8 get shrunk in the presence of LRF, severely deteriorating the CP-violation discovery reach of LBNO as can be seen from the right panel of figure 12. Table 7 also validates this result, where we compare the precise fraction of $\delta_{\mathrm{CP}}$ (true) for which a discovery is possible for CP-violation from LBNO (70 kt) and DUNE (35 kt) at $2 \sigma$ and $3 \sigma$ confidence levels. For LBNO set-up with true $\mathrm{NH}$ and $\alpha_{e \mu}$ (true) $=10^{-52}$, the coverage in $\delta_{\mathrm{CP}}$ (true) at $3 \sigma$ C.L. reduces to $30 \%$ from $55 \%$ as we have in the 'SM' case. In case of true IH, the impact of long-range potential is even more dramatic for these future facilities. At $3 \sigma$ with $\alpha_{e \mu}$ (true) $=10^{-52}$, their CP-violation reach is quite minimal: only $12 \%$ for LBNO and $37 \%$ for DUNE while in the 'SM' framework, the coverage is $60 \%$ for LBNO and $53 \%$ for DUNE. Since, the sign of the long-range potential $V_{e \mu}$ is opposite for neutrino and anti-neutrino, it affects the neutrino and anti-neutrino oscillation probabilities in different fashion. This feature introduces fake CP-asymmetry like the SM matter effect and severely limits the $\mathrm{CP}$-violation search in these long-baseline facilities which can be clearly seen from table 7 .

Finally, to see the complete picture, the fraction of $\delta_{\mathrm{CP}}$ (true) for which a discovery is possible for CP-violation is shown in figure 13 as a function of true value of $\alpha_{e \mu}$ assuming $\mathrm{NH}$ as true hierarchy. In each panel, we compare the performances of DUNE (35 kt) and LBNO (70 kt) which are shown by red and blue lines respectively. We give the results at $2 \sigma$ (left panel) and $3 \sigma$ (right panel) confidence levels. In both the panels, the solid horizontal lines depict the 'SM' case where $\alpha_{e \mu}=0$ in the data and also in the fit. For the 'SM+LRF' case (dashed lines), the data is generated with the true value of $\alpha_{e \mu}$ as shown in the x-axis and in the fit, we marginalize over test values of $\alpha_{e \mu}$ in its allowed range. The rest of the simulation details are exactly similar to the 'SM' case as discussed before. For the values close to $\alpha_{e \mu}$ (true) $=10^{-54}$, the event spectra in the data is almost similar to the 'SM' case, 

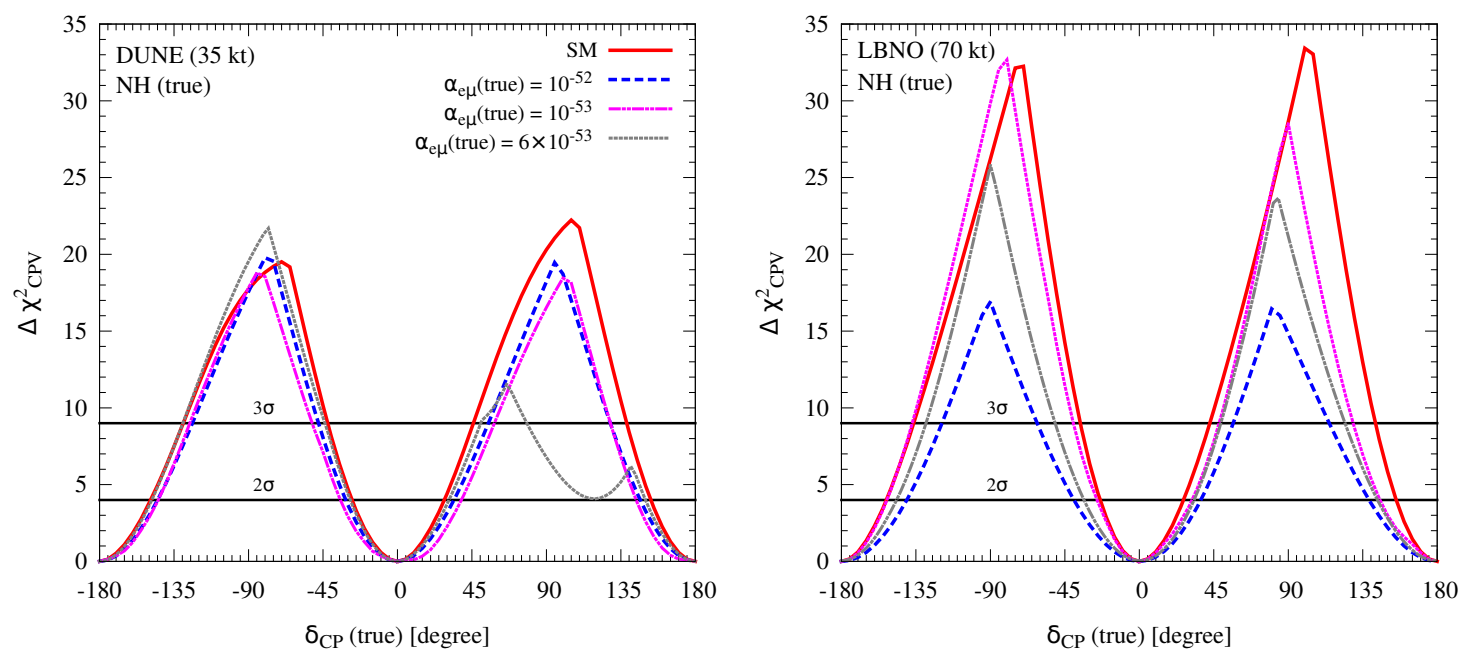

Figure 12. CP-violation discovery reach as a function of true value of $\delta_{\mathrm{CP}}$ assuming $\mathrm{NH}$ as true hierarchy. In the left panel, we show the results for DUNE (35 kt) and the right panel is for LBNO $(70 \mathrm{kt})$. For the 'SM' case, $\alpha_{e \mu}=0$ in the data and also in the fit. For each $\delta_{\mathrm{CP}}($ true), we also give the results generating the data with three different true values of $\alpha_{e \mu}$ which are mentioned in the figure legends. In all these three cases, in the fit, we marginalize over test values of $\alpha_{e \mu}$ in its allowed range. The rest of the simulation details are exactly similar to the 'SM' case (see text for details).

\begin{tabular}{|c|c||c|c||c|c|}
\hline \multicolumn{2}{|c||}{ True Hierarchy } & \multicolumn{2}{c||}{ DUNE (35 kt) } & \multicolumn{2}{c|}{ LBNO (70 kt) } \\
\cline { 3 - 6 } & SM & $\alpha_{e \mu}($ true $)=10^{-52}$ & SM & $\alpha_{e \mu}($ true $)=10^{-52}$ \\
\hline \multirow{2}{*}{$2 \sigma$ C.L. } & NH (true) & 0.67 & 0.62 & 0.71 & 0.56 \\
& IH (true) & 0.68 & 0.59 & 0.73 & 0.44 \\
\hline \multirow{2}{*}{$3 \sigma$ C.L. } & NH (true) & 0.48 & 0.41 & 0.55 & 0.30 \\
& IH (true) & 0.53 & 0.37 & 0.60 & 0.12 \\
\hline
\end{tabular}

Table 7. Fraction of $\delta_{\mathrm{CP}}$ (true) for which a discovery is possible for CP-violation from DUNE (35 kt) and LBNO (70 kt) set-ups at $2 \sigma$ and $3 \sigma$ confidence levels. We show the coverage in $\delta_{\mathrm{CP}}$ (true) for both the choices of true hierarchy: $\mathrm{NH}$ and IH. For the 'SM' cases, we consider $\alpha_{e \mu}=0$ in the data and also in the fit. We also give the results generating the data with $\alpha_{e \mu}$ (true) $=10^{-52}$ and in the fit, we marginalize over test values of $\alpha_{e \mu}$ in its allowed range. The rest of the simulation details are exactly similar to the 'SM' case (see text for details).

but since we allow $\alpha_{e \mu}$ to vary in the fit in the range $10^{-54}$ to $10^{-51}$ along with the other three-flavor oscillation parameters as discussed before, we see a small suppression in the fraction of $\delta_{\mathrm{CP}}$ (true). In both the panels, around $\alpha_{e \mu}($ true $)=6 \times 10^{-53}$, the CP-violation discovery reach of DUNE deteriorates substantially, which we also observe in figure 12, and the marginalization over $\theta_{23}$ is mainly responsible for this as we have already discussed. Once $\alpha_{e \mu}$ (true) approaches toward $10^{-52}$, the coverages in $\delta_{\mathrm{CP}}$ (true) for which CP-violation can be observed, shrink very rapidly for both the set-ups, and ultimately around $\alpha_{e \mu}$ (true) $=2 \times 10^{-52}$, the coverages almost become zero. We can understand this feature from our 

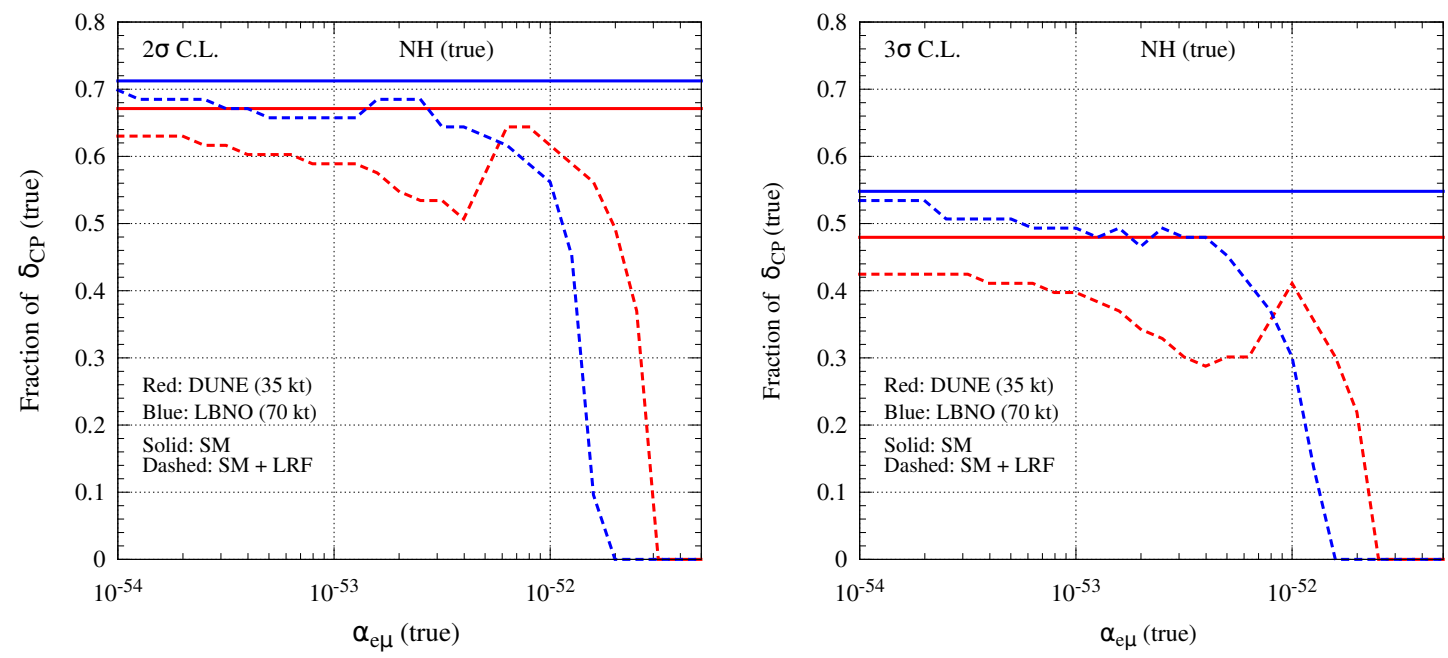

Figure 13. Fraction of $\delta_{\mathrm{CP}}$ (true) for which a discovery is possible for CP-violation is plotted as a function of true value of $\alpha_{e \mu}$ assuming $\mathrm{NH}$ as true hierarchy. In each panel, we compare the performances of DUNE (35 kt) and LBNO (70 kt) which are shown by red and blue lines respectively. We give the results at $2 \sigma$ (left panel) and $3 \sigma$ (right panel) confidence levels. In both the panels, the solid lines portray the 'SM' scenario where $\alpha_{e \mu}=0$ in the data and also in the fit. For the 'SM+LRF' case (dashed lines), the data is generated with the true value of $\alpha_{e \mu}$ as shown in the x-axis and in the fit, we marginalize over test values of $\alpha_{e \mu}$ in its allowed range. The rest of the simulation details are exactly similar to the 'SM' case (see text for details).

discussions in section 3.1, where we have seen that in the presence of $V_{e \mu}$, as we increase $E, \theta_{13}^{m}$ quickly approaches toward $45^{\circ}$ (see middle panel of figure 1), and the resonance occurs at much lower energies as compared to the SM case. Finally, $\theta_{13}^{m}$ reaches to $90^{\circ}$ as we further increase $E$, and the $\nu_{\mu} \rightarrow \nu_{e}$ oscillation probabilities vanish for most of the energies where we have significant amount of neutrino flux. It causes a huge suppression in the event rates and as a result, the sensitivity goes to zero. Next, we turn our attention to the mass hierarchy discovery potential of DUNE and LBNO.

\subsection{Impact of LRF on mass hierarchy measurements}

The large Earth matter effects at both the DUNE and LBNO baselines enhance the separation between the oscillation spectra of $\mathrm{NH}$ and $\mathrm{IH}$, and hence, we have large differences in the event rates for $\mathrm{NH}$ and $\mathrm{IH}$, leading to unprecedented sensitivity toward neutrino mass hierarchy. Now, it would be quite interesting to see how robust are these measurements in the presence of LRF? A 'discovery' of the mass hierarchy is a discrete measurement and is defined as the ability to exclude any degenerate solution for the wrong (fit) hierarchy at a given confidence level. For hierarchy sensitivity, we first assume NH to be the true hierarchy and we choose a true value of $\delta_{\mathrm{CP}}$ and $\alpha_{e \mu}$. We compute the NH event spectrum for these assumptions and the other true values of the oscillation parameters (see the third column of table 2) and label it to be data. Then, we estimate the various theoretical event spectra assuming IH and a test value of $\alpha_{e \mu}$ as shown in the x-axis of figure 14, and by 
varying simultaneously test $\theta_{23}$ in its $3 \sigma$ allowed range and test $\delta_{\mathrm{CP}}$ in the full allowed range $\left(-180^{\circ}\right.$ to $\left.180^{\circ}\right)$. Next, we compute the $\Delta \chi^{2}$ between each set of predicted and theoretical event spectra using the numerical technique described in section 5 . The smallest of all such $\Delta \chi^{2}$ values: $\Delta \chi_{\mathrm{MH}}^{2}$ is shown in figure 14 as a function of $\alpha_{e \mu}$ (test) for given choices of $\alpha_{e \mu}$ (true) and $\delta_{\mathrm{CP}}$ (true). As mentioned above, we always assume $\mathrm{NH}$ in the data and $\mathrm{IH}$ in the fit while generating the curves in figure 14. The upper panels portray the performance of DUNE (35 kt), while the lower panels are for LBNO (70 kt). In each panel, the results are given for four different choices of $\delta_{\mathrm{CP}}($ true) and the solid horizontal lines depict the 'SM' case where $\alpha_{e \mu}$ is zero in the data and also in the fit. For the 'SM+LRF' case (dashed lines), the data is generated with the true value of $\alpha_{e \mu}$ as mentioned in the top part of each panel, and in the fit, we vary the test values of $\alpha_{e \mu}$ while marginalizing over $\theta_{23}$ and $\delta_{\mathrm{CP}}$. The rest of the simulation details are exactly similar to the 'SM' case as mentioned above. Though in figure 14, we have shown the results for three benchmark values of $\alpha_{e \mu}$ (true) $=10^{-53}$ (left panels), $10^{-52}$ (middle panels), and $10^{-51}$ (right panels), but, we have checked that for DUNE, the mass hierarchy sensitivity always stays above the standard expectations irrespective of $\delta_{\mathrm{CP}}$ (true) provided the true value of $\alpha_{e \mu}<5 \times 10^{-52}$. There is a large suppression in the appearance event rates when we generate the data with a true value of $\alpha_{e \mu}$ around $5 \times 10^{-52}$, and if we further increase the value of $\alpha_{e \mu}$ (true), the statistical strength of the data reduces very rapidly, and the sensitivity goes below the standard expectation. The upper right panel in figure 14 clearly shows this behavior. In case of LBNO, the mass hierarchy discovery reach never goes below the 'SM' value irrespective of $\delta_{\mathrm{CP}}$ (true) if the true choice of $\alpha_{e \mu}$ is smaller than $10^{-52}$. Once we consider the true value of $\alpha_{e \mu} \geq 10^{-52}$, the appearance event rates get reduced in data by considerable amount, causing a significant drop in the sensitivity which can be clearly seen from the lower middle and right panels in figure 14. In figure 15, we generate the data with IH and fit it with NH. We see almost similar behavior in all the panels of figure 15 as we have noticed in figure 14 .

\section{$7 \quad$ Summary and conclusions}

Flavor-dependent long-range leptonic forces mediated by the extremely light and neutral bosons associated with gauged $L_{e}-L_{\mu}$ or $L_{e}-L_{\tau}$ symmetries, constitute a minimal extension of the SM preserving its renormalizability and can lead to interesting phenomenological consequences. For an example, the electrons inside the Sun can generate a flavor-dependent long-range potential $V_{e \mu / e \tau}$ at the Earth surface which can give rise to non-trivial three neutrino mixing affects in terrestrial experiments, and could influence the neutrino propagation through matter. The sign of this potential is opposite for anti-neutrinos, and affects the neutrino and anti-neutrino oscillation probabilities in different fashion. This feature invokes fake CP-asymmetry like the SM matter effect and can severely affect the leptonic CP-violation searches in long-baseline experiments. In this paper for the first time, we have investigated in detail the possible impacts of these long-range flavor-diagonal neutral current interactions in the oscillations of neutrinos and anti-neutrinos in the context of future high-precision superbeam facilities, DUNE and LBNO. The key point here is that 

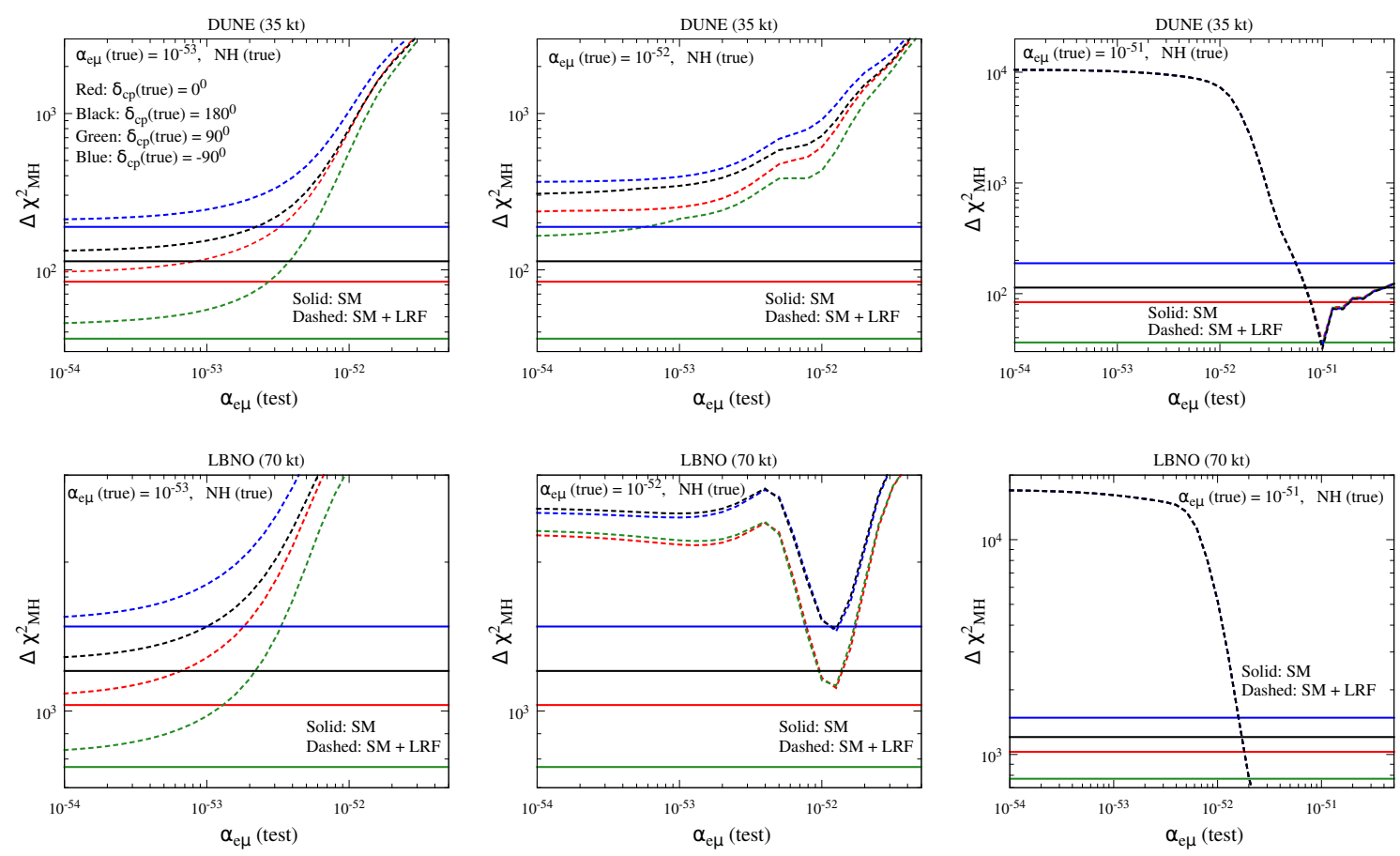

Figure 14. Discovery reach for mass hierarchy as a function of test $\alpha_{e \mu}$ assuming NH in the data and IH in the fit. The upper (lower) panels are for DUNE (LBNO). We give the results for four different true values of $\delta_{\mathrm{CP}}$ in each panel. The solid horizontal lines in each panel show the 'SM' case where $\alpha_{e \mu}=0$ in the data and also in the fit. For the 'SM+LRF' case (dashed lines), the data is generated with the true value of $\alpha_{e \mu}=10^{-53}$ (left panels), $10^{-52}$ (middle panels), and $10^{-51}$ (right panels). Then, in the fit, we vary the test values of $\alpha_{e \mu}$ while marginalizing over $\theta_{23}$ and $\delta_{\mathrm{CP}}$. The rest of the simulation details are exactly similar to the 'SM' case (see text for details). Also, note that the ranges in the $\mathrm{x}$-axis and $\mathrm{y}$-axis are different in some of the panels.

for long-baseline neutrinos, $\Delta m^{2} / 2 E \sim 2.5 \times 10^{-13} \mathrm{eV}$ (assuming $\Delta m^{2} \sim 2.5 \times 10^{-3} \mathrm{eV}^{2}$ and $E \sim 5 \mathrm{GeV}$ ) which is comparable to $V_{e \mu}$ even for $\alpha_{e \mu} \sim 10^{-52}$, and can influence the long-baseline experiments significantly. For the Fermilab-Homestake $(1300 \mathrm{~km})$ and CERNPyhäsalmi $\left(2290 \mathrm{~km}\right.$ ) baselines, the Earth matter potentials are also around $10^{-13} \mathrm{eV}$ (see table 1), suggesting that $V_{C C}$ can also interfere with $V_{e \mu}$ and $\Delta m_{31}^{2} / 2 E$, having substantial impact on the oscillation probability. We have explored these interesting possibilities in detail in this work. In this paper, we have presented all the results considering the $L_{e}-L_{\mu}$ symmetry. Similar analysis can be performed for the $L_{e}-L_{\tau}$ symmetry which we will present elsewhere.

We have derived approximate analytical expressions for the effective neutrino oscillation parameters to study how they 'run' as functions of the neutrino energy in the presence of both long-range and Earth matter potentials. We have also obtained a compact and simple expression for the resonance energy, where $\theta_{13}$ becomes $45^{\circ}$ in the presence of both $V_{C C}$ and $V_{e \mu}$. We have observed that in the presence of $V_{e \mu}$, as we increase the neutrino energy, $\theta_{13}$ in matter quickly approaches toward $45^{\circ}$, and the resonance occurs at much 

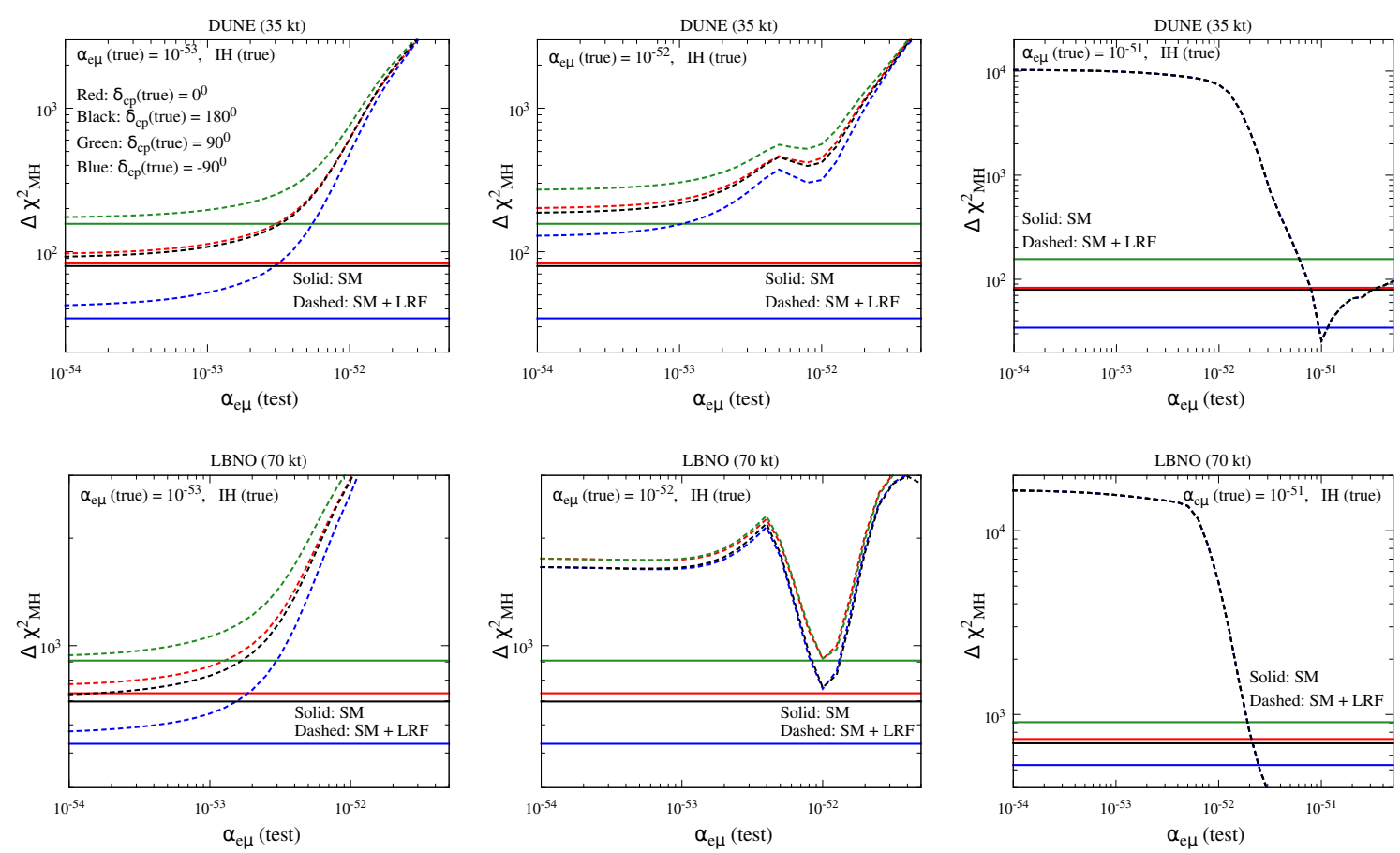

Figure 15. Discovery reach for mass hierarchy as a function of test $\alpha_{e \mu}$ assuming IH in the data and NH in the fit. The upper (lower) panels are for DUNE (LBNO). We give the results for four different true values of $\delta_{\mathrm{CP}}$ in each panel. The solid horizontal lines in each panel show the 'SM' case where $\alpha_{e \mu}=0$ in the data and also in the fit. For the 'SM+LRF' case (dashed lines), the data is generated with the true value of $\alpha_{e \mu}=10^{-53}$ (left panels), $10^{-52}$ (middle panels), and $10^{-51}$ (right panels). Then, in the fit, we vary the test values of $\alpha_{e \mu}$ while marginalizing over $\theta_{23}$ and $\delta_{\mathrm{CP}}$. The rest of the simulation details are exactly similar to the 'SM' case (see text for details). Also, note that the ranges in the $\mathrm{x}$-axis and $\mathrm{y}$-axis are different in some of the panels.

lower energies as compared to the SM case. Finally, $\theta_{13}$ in matter reaches to $90^{\circ}$ as we further increase the energy, causing a large suppression in the appearance probability for most of the energies where we have significant amount of neutrino flux for both the set-ups. As a result, the event rates get reduced which can be clearly seen from the bi-events plot in figure 8.

As the long-range potential due to gauged $L_{e}-L_{\mu}$ symmetry can change the standard oscillation picture of these future facilities significantly, we can expect to place strong constraints on $\alpha_{e \mu}$ if these experiments do not observe a signal of LRF in oscillations. For an example, if $\delta_{\mathrm{CP}}$ (true) is $-90^{\circ}$ and true hierarchy is $\mathrm{NH}$, then the expected bound from the DUNE (35 kt) set-up at $90 \%$ C.L. is $\alpha_{e \mu}<1.9 \times 10^{-53}$. The same from the LBNO (70 kt) experiment is $\alpha_{e \mu}<7.8 \times 10^{-54}$, suggesting that the constraint from LBNO is 2.4 times better than DUNE. This future limit from the DUNE (LBNO) experiment is almost 30 (70) times better than the existing bound from the SK experiment [85]. We have noticed that these future limits on $\alpha_{e \mu}$ from DUNE and LBNO are not very sensitive to the true choice of $\delta_{\mathrm{CP}}$ and mass hierarchy. We have also estimated the discovery reach for $\alpha_{e \mu}$ if we 
find a positive signal of LRF in the expected event spectra at DUNE and LBNO. We have found that the spectral information on the signal and background events is quite crucial to constrain/discover this new long-range force.

We have also studied in detail the CP-violation discovery reach of DUNE (35 kt) and LBNO (70 kt) in the presence of LRF. We have seen that the CP-violation measurements can be deteriorated by considerable amount as compared to the standard expectation depending on the true value of $\alpha_{e \mu}$. At $3 \sigma$ with $\alpha_{e \mu}$ (true) $=10^{-52}$ and true NH, the coverage in $\delta_{\mathrm{CP}}$ (true) for which a discovery is possible for CP-violation is $41 \%$ (30\%) for DUNE (LBNO) while in the standard case, the coverage is $48 \%$ for DUNE and $55 \%$ for LBNO. In case of true IH, the impact of long-range potential is even more striking for these future facilities. As an example, if $\alpha_{e \mu}$ (true) $=10^{-52}$, their chances of establishing CP-violation are quite minimal: only for $37 \%(12 \%)$ values of $\delta_{\mathrm{CP}}$ (true), DUNE (LBNO) can reject both the $\mathrm{CP}$-conserving values $0^{\circ}$ and $180^{\circ}$ in the fit at $3 \sigma$, while in the 'SM' framework, DUNE (LBNO) can do so for $53 \%$ (60\%) values of true $\delta_{\mathrm{CP}}$. As the true value of $\alpha_{e \mu}$ approaches toward $10^{-52}$, the coverages in $\delta_{\mathrm{CP}}$ (true) for which CP-violation can be observed, diminish very quickly for both the set-ups, and ultimately around $\alpha_{e \mu}$ (true) $=$ $2 \times 10^{-52}$, the coverages almost become zero.

Finally, we have asked the question, how robust are mass hierarchy measurements in these future facilities in the presence of LRF? In the standard case, due to the large Earth matter effects at the Fermilab-Homestake and CERN-Pyhäsalmi baselines, both DUNE and LBNO can resolve the issue of mass hierarchy at very high confidence level. Now, if LRF exists in Nature, then for DUNE, the mass hierarchy sensitivity remains above the standard expectations provided the true value of $\alpha_{e \mu}<5 \times 10^{-52}$. In case of LBNO, the mass hierarchy discovery reach does not go below the 'SM' value as long as the true value of $\alpha_{e \mu}$ is smaller than $10^{-52}$.

\section{Acknowledgments}

S.K.A. would like to thank Anjan S. Joshipura and Subhendra Mohanty for useful discussions on long-range forces. S.K.A. was supported by the DST/INSPIRE Research Grant [IFA-PH-12], Department of Science \& Technology, India. S.K.A. is grateful to the Mainz Institute for Theoretical Physics (MITP) for its hospitality and its partial support during the completion of this work. S.S.C. would like to thank the organizers of the XXI DAE-BRNS High Energy Physics Symposium 2014 for giving an opportunity to present the preliminary results of this work.

\section{A Discussion at the probability level - anti-neutrino case}

In figure 16, we plot the exact numerical transition probability $\bar{\nu}_{\mu} \rightarrow \bar{\nu}_{e}$ as a function of anti-neutrino energy. The band shows the impact of unknown $\delta_{\mathrm{CP}}$. Inside each band, the probability for $\delta_{\mathrm{CP}}=0^{\circ}$ case is shown by the black dashed line. The left panels (right panels) are drawn for $1300 \mathrm{~km}(2290 \mathrm{~km})$ baseline. In each panel, we compare the 

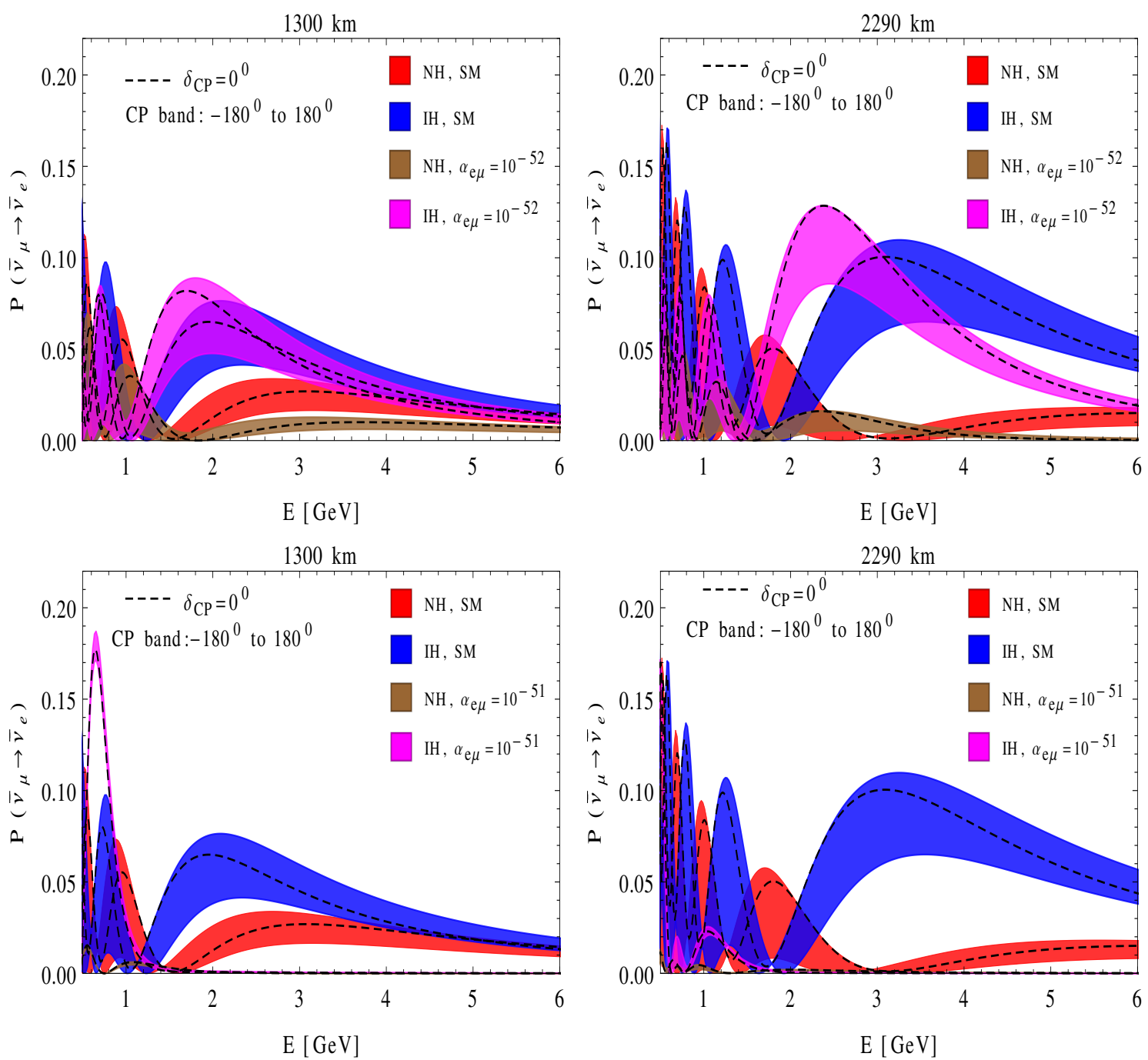

Figure 16. The transition probability $P_{\bar{\mu} \bar{e}}$ as a function of anti-neutrino energy. The band reflects the effect of unknown $\delta_{\mathrm{CP}}$. Inside each band, the probability for $\delta_{\mathrm{CP}}=0^{\circ}$ case is shown by the black dashed line. The left panels (right panels) are for $1300 \mathrm{~km}(2290 \mathrm{~km})$ baseline. In each panel, we compare the probabilities for $\mathrm{NH}$ and $\mathrm{IH}$ with and without long-range potential. In the upper (lower) panels, we take $\alpha_{e \mu}=10^{-52}\left(\alpha_{e \mu}=10^{-51}\right)$ for the cases with long-range potential.

probabilities for $\mathrm{NH}$ and $\mathrm{IH}$ with and without long-range potential. In the upper (lower) panels, we consider $\alpha_{e \mu}=10^{-52}\left(\alpha_{e \mu}=10^{-51}\right)$ for the cases with long-range potential.

Figure 17 shows the exact numerical $\bar{\nu}_{\mu} \rightarrow \bar{\nu}_{\mu}$ disappearance probability as a function of anti-neutrino energy. The thin band portrays the mild impact of unknown $\delta_{\mathrm{CP}}$. Inside each band, the probability for $\delta_{\mathrm{CP}}=0^{\circ}$ case is given by the black dashed line. The left panels (right panels) are drawn for $1300 \mathrm{~km}(2290 \mathrm{~km})$ baseline. In each panel, we compare the probabilities for $\mathrm{NH}$ and $\mathrm{IH}$ with and without long-range potential. In the upper (lower) panels, we consider $\alpha_{e \mu}=10^{-52}\left(\alpha_{e \mu}=10^{-51}\right)$ for the cases with long-range potential. 

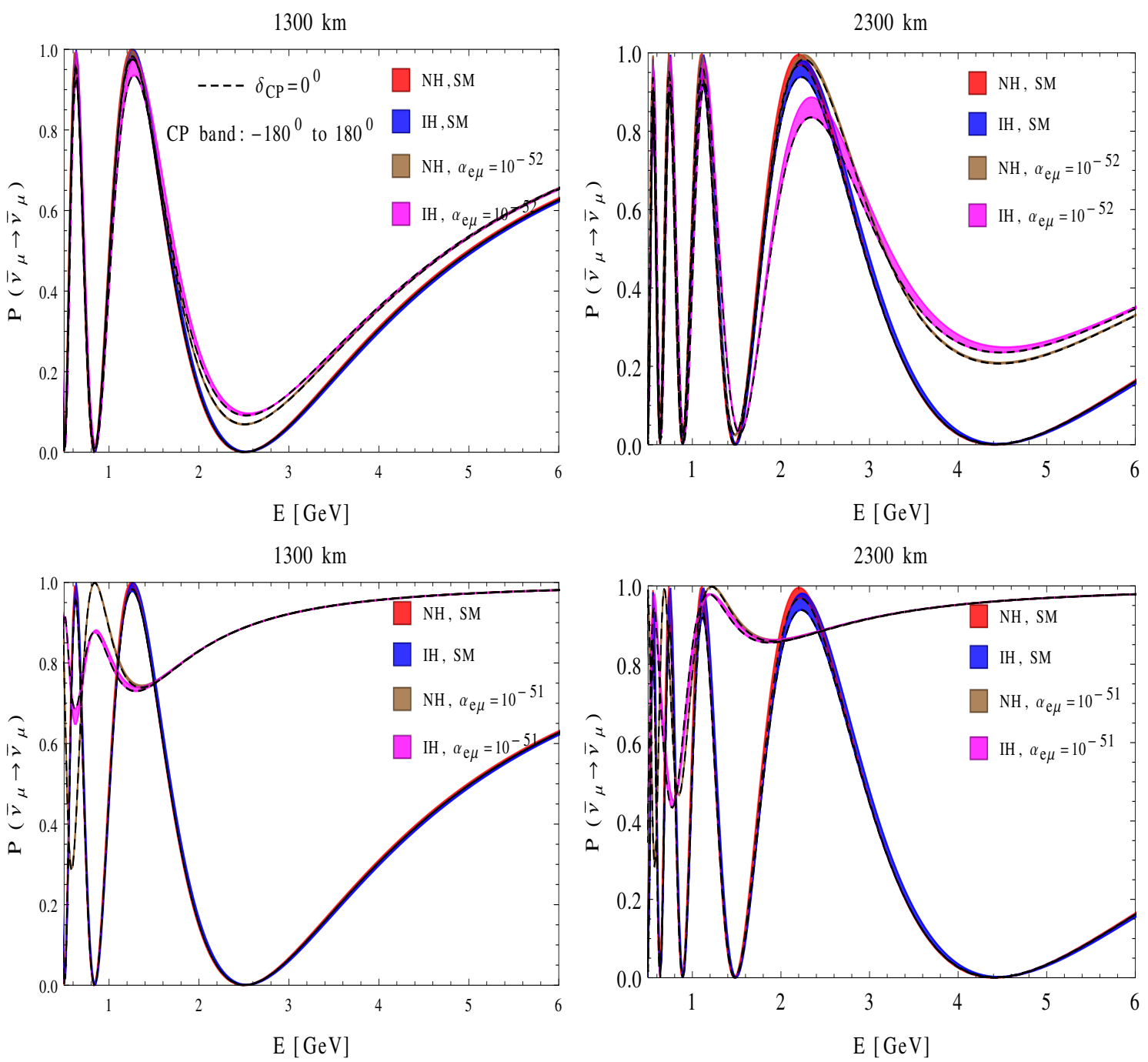

Figure 17. The transition probability $P_{\bar{\mu} \bar{\mu}}$ as a function of anti-neutrino energy. The band reflects the effect of unknown $\delta_{\mathrm{CP}}$. Inside each band, the probability for $\delta_{\mathrm{CP}}=0^{\circ}$ case is shown by the black dashed line. The left panels (right panels) are for $1300 \mathrm{~km}(2290 \mathrm{~km})$ baseline. In each panel, we compare the probabilities for $\mathrm{NH}$ and $\mathrm{IH}$ with and without long-range potential. In the upper (lower) panels, we take $\alpha_{e \mu}=10^{-52}\left(\alpha_{e \mu}=10^{-51}\right)$ for the cases with long-range potential.

Open Access. This article is distributed under the terms of the Creative Commons Attribution License (CC-BY 4.0), which permits any use, distribution and reproduction in any medium, provided the original author(s) and source are credited.

\section{References}

[1] R.N. Mohapatra et al., Theory of neutrinos: A White paper, Rept. Prog. Phys. 70 (2007) 1757 [hep-ph/0510213] [INSPIRE].

[2] A. Strumia and F. Vissani, Neutrino masses and mixings and..., hep-ph/0606054 [INSPIRE]. 
[3] M.C. Gonzalez-Garcia and M. Maltoni, Phenomenology with Massive Neutrinos, Phys. Rept. 460 (2008) 1 [arXiv:0704.1800] [INSPIRE].

[4] Planck collaboration, P.A.R. Ade et al., Planck 2013 results. XVI. Cosmological parameters, Astron. Astrophys. 571 (2014) A16 [arXiv:1303.5076] [InSPIRE].

[5] F.T. Avignone, III, S.R. Elliott and J. Engel, Double Beta Decay, Majorana Neutrinos and Neutrino Mass, Rev. Mod. Phys. 80 (2008) 481 [arXiv:0708.1033] [InSPIRE].

[6] J.D. Vergados, H. Ejiri and F. Simkovic, Theory of Neutrinoless Double Beta Decay, Rept. Prog. Phys. 75 (2012) 106301 [arXiv: 1205.0649] [INSPIRE].

[7] W. Rodejohann, Neutrinoless double beta decay and neutrino physics, J. Phys. G 39 (2012) 124008 [arXiv: 1206.2560] [INSPIRE].

[8] H. Päs and W. Rodejohann, Neutrinoless Double Beta Decay, arXiv:1507.00170 [INSPIRE].

[9] S.M. Bilenky, Neutrino oscillations: brief history and present status, arXiv:1408.2864 [INSPIRE].

[10] I. Gil-Botella, Neutrino Physics, arXiv:1504.03551 [INSPIRE].

[11] Y. Wang and Z.-z. Xing, Neutrino Masses and Flavor Oscillations, arXiv:1504.06155 [INSPIRE].

[12] B. Pontecorvo, Neutrino Experiments and the Problem of Conservation of Leptonic Charge, Sov. Phys. JETP 26 (1968) 984 [inSPIRE].

[13] V.N. Gribov and B. Pontecorvo, Neutrino astronomy and lepton charge, Phys. Lett. B 28 (1969) 493 [INSPIRE].

[14] Particle Data Group collaboration, K.A. Olive et al., Review of Particle Physics, Chin. Phys. C 38 (2014) 090001 [inSPIRE].

[15] J. Hewett et al., Fundamental Physics at the Intensity Frontier, arXiv:1205.2671 [INSPIRE].

[16] B.T. Cleveland et al., Measurement of the solar electron neutrino flux with the Homestake chlorine detector, Astrophys. J. 496 (1998) 505 [INSPIRE].

[17] GNO collaboration, M. Altmann et al., Complete results for five years of GNO solar neutrino observations, Phys. Lett. B 616 (2005) 174 [hep-ex/0504037] [INSPIRE].

[18] Super-Kamiokande collaboration, J. Hosaka et al., Solar neutrino measurements in Super-Kamiokande-I, Phys. Rev. D 73 (2006) 112001 [hep-ex/0508053] [InSPIRE].

[19] SNO collaboration, Q.R. Ahmad et al., Direct evidence for neutrino flavor transformation from neutral current interactions in the Sudbury Neutrino Observatory, Phys. Rev. Lett. 89 (2002) 011301 [nucl-ex/0204008] [INSPIRE].

[20] SNO collaboration, B. Aharmim et al., An Independent Measurement of the Total Active B-8 Solar Neutrino Flux Using an Array of He-3 Proportional Counters at the Sudbury Neutrino Observatory, Phys. Rev. Lett. 101 (2008) 111301 [arXiv:0806.0989] [INSPIRE].

[21] SNO collaboration, B. Aharmim et al., Low Energy Threshold Analysis of the Phase I and Phase II Data Sets of the Sudbury Neutrino Observatory, Phys. Rev. C 81 (2010) 055504 [arXiv:0910.2984] [INSPIRE]. 
[22] Borexino collaboration, C. Arpesella et al., Direct Measurement of the Be-7 Solar Neutrino Flux with 192 Days of Borexino Data, Phys. Rev. Lett. 101 (2008) 091302 [arXiv:0805.3843] [INSPIRE].

[23] Super-Kamiokande collaboration, Y. Fukuda et al., Evidence for oscillation of atmospheric neutrinos, Phys. Rev. Lett. 81 (1998) 1562 [hep-ex/9807003] [InSPIRE].

[24] Super-Kamiokande collaboration, Y. Ashie et al., A Measurement of atmospheric neutrino oscillation parameters by Super-Kamiokande I, Phys. Rev. D 71 (2005) 112005 [hep-ex/0501064] [INSPIRE].

[25] Super-Kamiokande collaboration, R. Wendell et al., Atmospheric neutrino oscillation analysis with sub-leading effects in Super-Kamiokande I, II and III, Phys. Rev. D 81 (2010) 092004 [arXiv: 1002 . 3471] [INSPIRE].

[26] KamLAND collaboration, T. Araki et al., Measurement of neutrino oscillation with KamLAND: Evidence of spectral distortion, Phys. Rev. Lett. 94 (2005) 081801 [hep-ex/0406035] [INSPIRE].

[27] KamLAND collaboration, S. Abe et al., Precision Measurement of Neutrino Oscillation Parameters with KamLAND, Phys. Rev. Lett. 100 (2008) 221803 [arXiv:0801.4589] [INSPIRE].

[28] DAYA BAY collaboration, F.P. An et al., Observation of electron-antineutrino disappearance at Daya Bay, Phys. Rev. Lett. 108 (2012) 171803 [arXiv:1203.1669] [INSPIRE].

[29] DaYa BAY collaboration, F.P. An et al., Improved Measurement of Electron Antineutrino Disappearance at Daya Bay, Chin. Phys. C 37 (2013) 011001 [arXiv:1210.6327] [INSPIRE].

[30] DAYA BAY collaboration, F.P. An et al., Spectral measurement of electron antineutrino oscillation amplitude and frequency at Daya Bay, Phys. Rev. Lett. 112 (2014) 061801 [arXiv: 1310.6732] [INSPIRE].

[31] Daya Bay collaboration, F.P. An et al., A new Measurement of Antineutrino Oscillation with the Full Detector Configuration at Daya Bay, Phys. Rev. Lett. 115 (2015) 111802 [arXiv: 1505. 03456] [INSPIRE].

[32] RENO collaboration, J.K. Ahn et al., Observation of Reactor Electron Antineutrino Disappearance in the RENO Experiment, Phys. Rev. Lett. 108 (2012) 191802 [arXiv:1204.0626] [INSPIRE].

[33] Double CHOOZ collaboration, Y. Abe et al., Indication for the disappearance of reactor electron antineutrinos in the Double CHOOZ experiment, Phys. Rev. Lett. 108 (2012) 131801 [arXiv: 1112.6353] [INSPIRE].

[34] Double CHOOZ collaboration, Y. Abe et al., Reactor electron antineutrino disappearance in the Double CHOOZ experiment, Phys. Rev. D 86 (2012) 052008 [arXiv:1207.6632] [INSPIRE].

[35] Double CHOOZ collaboration, Y. Abe et al., First Measurement of $\theta_{13}$ from Delayed Neutron Capture on Hydrogen in the Double CHOOZ Experiment, Phys. Lett. B 723 (2013) 66 [arXiv: 1301.2948] [INSPIRE].

[36] Double CHOOZ collaboration, Y. Abe et al., Improved measurements of the neutrino mixing angle $\theta_{13}$ with the Double CHOOZ detector, JHEP 10 (2014) 086 [Erratum ibid. 1502 (2015) 074] [arXiv: 1406.7763] [INSPIRE]. 
[37] K2K collaboration, M.H. Ahn et al., Measurement of Neutrino Oscillation by the K2K Experiment, Phys. Rev. D 74 (2006) 072003 [hep-ex/0606032] [INSPIRE].

[38] MINOS collaboration, P. Adamson et al., Measurement of Neutrino Oscillations with the MINOS Detectors in the NuMI Beam, Phys. Rev. Lett. 101 (2008) 131802 [arXiv:0806.2237] [INSPIRE].

[39] MINOS collaboration, P. Adamson et al., Improved search for muon-neutrino to electron-neutrino oscillations in MINOS, Phys. Rev. Lett. 107 (2011) 181802 [arXiv:1108.0015] [INSPIRE].

[40] MINOS collaboration, P. Adamson et al., Electron neutrino and antineutrino appearance in the full MINOS data sample, Phys. Rev. Lett. 110 (2013) 171801 [arXiv:1301.4581] [INSPIRE].

[41] MINOS collaboration, P. Adamson et al., Measurement of Neutrino and Antineutrino Oscillations Using Beam and Atmospheric Data in MINOS, Phys. Rev. Lett. 110 (2013) 251801 [arXiv: 1304.6335] [INSPIRE].

[42] T2K collaboration, K. Abe et al., Indication of Electron Neutrino Appearance from an Accelerator-produced Off-axis Muon Neutrino Beam, Phys. Rev. Lett. 107 (2011) 041801 [arXiv:1106.2822] [INSPIRE].

[43] T2K collaboration, K. Abe et al., Evidence of Electron Neutrino Appearance in a Muon Neutrino Beam, Phys. Rev. D 88 (2013) 032002 [arXiv:1304.0841] [InSPIRE].

[44] T2K collaboration, K. Abe et al., Observation of Electron Neutrino Appearance in a Muon Neutrino Beam, Phys. Rev. Lett. 112 (2014) 061802 [arXiv:1311.4750] [INSPIRE].

[45] T2K collaboration, K. Abe et al., Precise Measurement of the Neutrino Mixing Parameter $\theta_{23}$ from Muon Neutrino Disappearance in an Off-Axis Beam, Phys. Rev. Lett. 112 (2014) 181801 [arXiv: 1403.1532] [INSPIRE].

[46] T2K collaboration, K. Abe et al., Measurements of neutrino oscillation in appearance and disappearance channels by the T2K experiment with $6.6 \times 10^{20}$ protons on target, Phys. Rev. D 91 (2015) 072010 [arXiv:1502.01550] [InSPIRE].

[47] B. Pontecorvo, Inverse beta-processes and non-conservation of lepton charge, Sov. Phys. JETP 7 (1958) 172.

[48] Z. Maki, M. Nakagawa and S. Sakata, Remarks on the unified model of elementary particles, Prog. Theor. Phys. 28 (1962) 870 [inSPIRE].

[49] S. Pascoli and T. Schwetz, Prospects for neutrino oscillation physics, Adv. High Energy Phys. 2013 (2013) 503401.

[50] S.K. Agarwalla, S. Prakash and S. Uma Sankar, Exploring the three flavor effects with future superbeams using liquid argon detectors, JHEP 03 (2014) 087 [arXiv:1304.3251] [INSPIRE].

[51] S.K. Agarwalla, Physics Potential of Long-Baseline Experiments, Adv. High Energy Phys. 2014 (2014) 457803 [arXiv:1401.4705] [INSPIRE].

[52] M.C. Gonzalez-Garcia, M. Maltoni and T. Schwetz, Updated fit to three neutrino mixing: status of leptonic CP-violation, JHEP 11 (2014) 052 [arXiv:1409.5439] [INSPIRE].

[53] F. Capozzi, G.L. Fogli, E. Lisi, A. Marrone, D. Montanino and A. Palazzo, Status of three-neutrino oscillation parameters, circa 2013, Phys. Rev. D 89 (2014) 093018 [arXiv: 1312.2878] [INSPIRE]. 
[54] D.V. Forero, M. Tortola and J.W.F. Valle, Neutrino oscillations refitted, Phys. Rev. D 90 (2014) 093006 [arXiv: 1405.7540] [INSPIRE].

[55] K.N. Abazajian et al., Light Sterile Neutrinos: A White Paper, arXiv:1204.5379 [INSPIRE].

[56] L. Wolfenstein, Neutrino Oscillations in Matter, Phys. Rev. D 17 (1978) 2369 [InSPIRE].

[57] S.P. Mikheev and A. Yu. Smirnov, Resonance Amplification of Oscillations in Matter and Spectroscopy of Solar Neutrinos, Sov. J. Nucl. Phys. 42 (1985) 913 [InSPIRE].

[58] S.P. Mikheev and A. Yu. Smirnov, Resonant amplification of neutrino oscillations in matter and solar neutrino spectroscopy, Nuovo Cim. C 9 (1986) 17 [InSPIRE].

[59] M. Blennow and A. Yu. Smirnov, Neutrino propagation in matter, Adv. High Energy Phys. 2013 (2013) 972485 [arXiv:1306.2903] [INSPIRE].

[60] G.J. Feldman, J. Hartnell and T. Kobayashi, Long-baseline neutrino oscillation experiments, Adv. High Energy Phys. 2013 (2013) 475749 [arXiv:1210.1778] [INSPIRE].

[61] G.L. Fogli and E. Lisi, Tests of three flavor mixing in long baseline neutrino oscillation experiments, Phys. Rev. D 54 (1996) 3667 [hep-ph/9604415] [INSPIRE].

[62] T2K collaboration, Y. Itow et al., The JHF-Kamioka neutrino project, hep-ex/0106019 [INSPIRE].

[63] T2K collaboration, K. Abe et al., The T2K Experiment, Nucl. Instrum. Meth. A 659 (2011) 106 [arXiv: 1106.1238] [INSPIRE].

[64] D. Ayres et al., Letter of Intent to build an Off-axis Detector to study numu to nue oscillations with the NuMI Neutrino Beam, hep-ex/0210005 [INSPIRE].

[65] NOvA collaboration, D.S. Ayres et al., NOvA: Proposal to build a 30 kiloton off-axis detector to study $\nu_{\mu} \rightarrow \nu_{e}$ oscillations in the NuMI beamline, hep-ex/0503053 [INSPIRE].

[66] NOvA collaboration, D. Ayres et al., The NOvA Technical Design Report, FERMILAB-DESIGN-2007-01 (2007).

[67] P. Huber, M. Lindner, T. Schwetz and W. Winter, First hint for CP-violation in neutrino oscillations from upcoming superbeam and reactor experiments, JHEP 11 (2009) 044 [arXiv: 0907.1896] [INSPIRE].

[68] S.K. Agarwalla, S. Prakash, S.K. Raut and S.U. Sankar, Potential of optimized NOvA for large $\theta_{(13)}$ and combined performance with a LArTPC and T2K, JHEP 12 (2012) 075 [arXiv:1208.3644] [INSPIRE].

[69] S.K. Agarwalla, S. Prakash and S.U. Sankar, Resolving the octant of theta23 with T2K and NOvA, JHEP 07 (2013) 131 [arXiv:1301.2574] [INSPIRE].

[70] A. Chatterjee, P. Ghoshal, S. Goswami and S.K. Raut, Octant sensitivity for large $\theta_{13}$ in atmospheric and long baseline neutrino experiments, JHEP 06 (2013) 010 [arXiv: 1302.1370] [INSPIRE].

[71] P.A.N. Machado, H. Minakata, H. Nunokawa and R. Zukanovich Funchal, What can we learn about the lepton CP phase in the next 10 years?, JHEP 05 (2014) 109 [arXiv: 1307.3248] [INSPIRE].

[72] M. Ghosh, P. Ghoshal, S. Goswami and S.K. Raut, Evidence for leptonic CP phase from

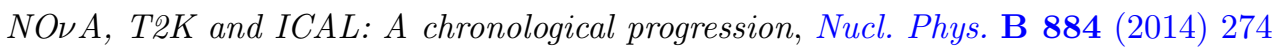
[arXiv: 1401.7243] [INSPIRE]. 
[73] T2K collaboration, K. Abe et al., Neutrino oscillation physics potential of the T2K experiment, PTEP 2015 (2015) 043C01 [arXiv:1409.7469] [INSPIRE].

[74] M.V. Diwan et al., Very long baseline neutrino oscillation experiments for precise measurements of mixing parameters and CP-violating effects, Phys. Rev. D 68 (2003) 012002 [hep-ph/0303081] [INSPIRE].

[75] V. Barger et al., Report of the US long baseline neutrino experiment study, arXiv:0705.4396 [INSPIRE].

[76] P. Huber and J. Kopp, Two experiments for the price of one? - The role of the second oscillation maximum in long baseline neutrino experiments, JHEP 03 (2011) 013 [Erratum ibid. 1105 (2011) 024] [arXiv: 1010.3706] [INSPIRE].

[77] LBNE collaboration, T. Akiri et al., The 2010 Interim Report of the Long-Baseline Neutrino Experiment Collaboration Physics Working Groups, arXiv:1110.6249 [INSPIRE].

[78] LBNE collaboration, C. Adams et al., The Long-Baseline Neutrino Experiment: Exploring Fundamental Symmetries of the Universe, arXiv:1307.7335 [INSPIRE].

[79] S.K. Agarwalla, T. Li and A. Rubbia, An Incremental approach to unravel the neutrino mass hierarchy and CP-violation with a long-baseline Superbeam for large $\theta_{13}$, JHEP 05 (2012) 154 [arXiv:1109.6526] [INSPIRE].

[80] A. Stahl et al., Expression of Interest for a very long baseline neutrino oscillation experiment (LBNO), CERN-SPSC-2012-021 (2012).

[81] LAGUNA-LBNO collaboration, S.K. Agarwalla et al., The mass-hierarchy and CP-violation discovery reach of the LBNO long-baseline neutrino experiment, JHEP 05 (2014) 094 [arXiv: 1312.6520] [INSPIRE].

[82] LAGUNA-LBNO collaboration, S.K. Agarwalla et al., Optimised sensitivity to leptonic $C P$-violation from spectral information: the $L B N O$ case at $2300 \mathrm{~km}$ baseline, arXiv: 1412.0593 [INSPIRE].

[83] V. Barger, D. Marfatia and K. Whisnant, Breaking eight fold degeneracies in neutrino CP-violation, mixing and mass hierarchy, Phys. Rev. D 65 (2002) 073023 [hep-ph/0112119] [INSPIRE].

[84] H. Minakata, H. Nunokawa and S.J. Parke, Parameter degeneracies in neutrino oscillation measurement of leptonic CP and T violation, Phys. Rev. D 66 (2002) 093012 [hep-ph/0208163] [INSPIRE].

[85] A.S. Joshipura and S. Mohanty, Constraints on flavor dependent long range forces from atmospheric neutrino observations at Super-Kamiokande, Phys. Lett. B 584 (2004) 103 [hep-ph/0310210] [INSPIRE].

[86] J.A. Grifols and E. Masso, Neutrino oscillations in the sun probe long range leptonic forces, Phys. Lett. B 579 (2004) 123 [hep-ph/0311141] [INSPIRE].

[87] M.C. Gonzalez-Garcia, P.C. de Holanda, E. Masso and R. Zukanovich Funchal, Probing long-range leptonic forces with solar and reactor neutrinos, JCAP 01 (2007) 005 [hep-ph/0609094] [INSPIRE].

[88] A. Bandyopadhyay, A.Dighe and A.S. Joshipura, Constraints on flavor-dependent long range forces from solar neutrinos and kamland, Phys. Rev. D 75 (2007) 093005 [hep-ph/0610263] [INSPIRE]. 
[89] A. Samanta, Long-range Forces: Atmospheric Neutrino Oscillation at a magnetized Detector, JCAP 09 (2011) 010 [arXiv:1001.5344] [INSPIRE].

[90] P. Langacker, The Physics of Heavy Z' Gauge Bosons, Rev. Mod. Phys. 81 (2009) 1199 [arXiv:0801.1345] [INSPIRE].

[91] R. Foot, New Physics From Electric Charge Quantization?, Mod. Phys. Lett. A 6 (1991) 527 [INSPIRE].

[92] X.-G. He, G.C. Joshi, H. Lew and R.R. Volkas, Simplest Z-prime model, Phys. Rev. D 44 (1991) 2118 [INSPIRE].

[93] R. Foot, X.G. He, H. Lew and R.R. Volkas, Model for a light Z-prime boson, Phys. Rev. D 50 (1994) 4571 [hep-ph/9401250] [INSPIRE].

[94] R. Foot, Avoiding the gauge hierarchy problem with see-sawed neutrino masses, Mod. Phys. Lett. A 20 (2005) 3035 [hep-ph/0505154] [INSPIRE].

[95] L3 collaboration, M. Acciarri et al., Search for manifestations of new physics in fermion pair production at LEP, Phys. Lett. B 489 (2000) 81 [hep-ex/0005028] [INSPIRE].

[96] OPAL collaboration, G. Abbiendi et al., Tests of the standard model and constraints on new physics from measurements of fermion pair production at 189-GeV to 209-GeV at LEP, Eur. Phys. J. C 33 (2004) 173 [hep-ex/0309053] [InSPIRE].

[97] DELPHI collaboration, J. Abdallah et al., Measurement and interpretation of fermion-pair production at LEP energies above the $Z$ resonance, Eur. Phys. J. C 45 (2006) 589 [hep-ex/0512012] [INSPIRE].

[98] ALEPH collaboration, S. Schael et al., Fermion pair production in $e^{+} e^{-}$collisions at 189-209-GeV and constraints on physics beyond the standard model, Eur. Phys. J. C 49 (2007) 411 [hep-ex/0609051] [INSPIRE].

[99] M. Honda, Y. Kao, N. Okamura, A. Pronin and T. Takeuchi, Constraints on New Physics from Long Baseline Neutrino Oscillation Experiments, arXiv:0707.4545 [INSPIRE].

[100] G. Dutta, A.S. Joshipura and K.B. Vijaykumar, Leptonic flavor violations in the presence of an extra Z, Phys. Rev. D 50 (1994) 2109 [hep-ph/9405292] [INSPIRE].

[101] J.G. Williams, S.G. Turyshev and D.H. Boggs, Progress in lunar laser ranging tests of relativistic gravity, Phys. Rev. Lett. 93 (2004) 261101 [gr-qc/0411113] [INSPIRE].

[102] J.G. Williams, X.X. Newhall and J.O. Dickey, Relativity parameters determined from lunar laser ranging, Phys. Rev. D 53 (1996) 6730 [INSPIRE].

[103] E.G. Adelberger, B.R. Heckel and A.E. Nelson, Tests of the gravitational inverse square law, Ann. Rev. Nucl. Part. Sci. 53 (2003) 77 [hep-ph/0307284] [InSPIRE].

[104] A.D. Dolgov, Long range forces in the universe, Phys. Rept. 320 (1999) 1 [INSPIRE].

[105] T.D. Lee and C.-N. Yang, Conservation of Heavy Particles and Generalized Gauge Transformations, Phys. Rev. 98 (1955) 1501 [INSPIRE].

[106] L.B. Okun, On muonic charge and muonic photons, Yad. Fiz. 10 (1969) 358 [INSPIRE].

[107] L. Okun, Leptons and photons, Phys. Lett. B 382 (1996) 389 [hep-ph/9512436] [InSPIRE].

[108] J.A. Grifols, E. Masso and S. Peris, Supernova neutrinos as probes of long range nongravitational interactions of dark matter, Astropart. Phys. 2 (1994) 161 [InSPIRE]. 
[109] J.A. Grifols, E. Masso and R. Toldra, Majorana neutrinos and long range forces, Phys. Lett. B 389 (1996) 563 [hep-ph/9606377] [INSPIRE].

[110] R. Horvat, Supernova MSW effect in the presence of leptonic long range forces, Phys. Lett. B 366 (1996) 241 [INSPIRE].

[111] S.K. Agarwalla, Y. Kao, D. Saha and T. Takeuchi, Running of Oscillation Parameters in Matter with Flavor-Diagonal Non-Standard Interactions of the Neutrino, JHEP 11 (2015) 035 [arXiv: 1506.08464$]$ [INSPIRE].

[112] J.N. Bahcall, Neutrino Astrophysics, Cambridge University Press, Cambridge U.K. (1989).

[113] J. Heeck and W. Rodejohann, Gauged $L_{\mu}-L_{\tau}$ and different Muon Neutrino and Anti-Neutrino Oscillations: MINOS and beyond, J. Phys. G 38 (2011) 085005 [arXiv: 1007.2655] [INSPIRE].

[114] H. Davoudiasl, H.-S. Lee and W.J. Marciano, Long-Range Lepton Flavor Interactions and Neutrino Oscillations, Phys. Rev. D 84 (2011) 013009 [arXiv:1102.5352] [INSPIRE].

[115] A.M. Dziewonski and D.L. Anderson, Preliminary reference earth model, Phys. Earth Planet. Inter. 25 (1981) 297.

[116] MINOS collaboration, R. Nichol, New results from MINOS, Nucl. Phys. Proc. Suppl. 235-236 (2013) 105.

[117] H. Nunokawa, S.J. Parke and R. Zukanovich Funchal, Another possible way to determine the neutrino mass hierarchy, Phys. Rev. D 72 (2005) 013009 [hep-ph/0503283] [INSPIRE].

[118] A. de Gouvêa, J. Jenkins and B. Kayser, Neutrino mass hierarchy, vacuum oscillations and vanishing -U(e3)—, Phys. Rev. D 71 (2005) 113009 [hep-ph/0503079] [INSPIRE].

[119] S.K. Agarwalla, Y. Kao and T. Takeuchi, Analytical approximation of the neutrino oscillation matter effects at large $\theta_{13}$, JHEP 04 (2014) 047 [arXiv: 1302.6773] [INSPIRE].

[120] Deep Underground Neutrino Experiment (DUNE), http://www.dunescience.org.

[121] M. Bishai, private communication (2012).

[122] S. di Luise, Optimization of Neutrino Fluxes for Future Long Baseline Neutrino Experiment, Poster presented at ICHEP2012 Conference, 4-11 July 2012, Melbourne, Australia, www.ichep2012.com.au/.

[123] M.D. Messier, Evidence for neutrino mass from observations of atmospheric neutrinos with Super-Kamiokande, Ph.D. Thesis, Boston University, Boston U.S.A. (1999).

[124] E.A. Paschos and J.Y. Yu, Neutrino interactions in oscillation experiments, Phys. Rev. D 65 (2002) 033002 [hep-ph/0107261] [INSPIRE].

[125] G. Zeller, private communication (2012).

[126] R. Petti and G. Zeller, Nuclear Effects in Water vs. Argon, Technical Report, LBNE docdb No. 740 .

[127] P. Huber, M. Lindner and W. Winter, Simulation of long-baseline neutrino oscillation experiments with GLoBES (General Long Baseline Experiment Simulator), Comput. Phys. Commun. 167 (2005) 195 [hep-ph/0407333] [INSPIRE].

[128] P. Huber, J. Kopp, M. Lindner, M. Rolinec and W. Winter, New features in the simulation of neutrino oscillation experiments with GLoBES 3.0: General Long Baseline Experiment Simulator, Comput. Phys. Commun. 177 (2007) 432 [hep-ph/0701187] [INSPIRE]. 
[129] Daya Bay collaboration, L. Zhan, Recent Results from Daya Bay, arXiv:1506.01149 [INSPIRE].

[130] S.K. Agarwalla, S. Prakash and W. Wang, High-precision measurement of atmospheric mass-squared splitting with T2K and NOvA, arXiv:1312.1477 [INSPIRE].

[131] P. Huber, M. Lindner and W. Winter, Superbeams versus neutrino factories, Nucl. Phys. B 645 (2002) 3 [hep-ph/0204352] [INSPIRE].

[132] G.L. Fogli, E. Lisi, A. Marrone, D. Montanino and A. Palazzo, Getting the most from the statistical analysis of solar neutrino oscillations, Phys. Rev. D 66 (2002) 053010 [hep-ph/0206162] [INSPIRE].

[133] M. Blennow, P. Coloma, P. Huber and T. Schwetz, Quantifying the sensitivity of oscillation experiments to the neutrino mass ordering, JHEP 03 (2014) 028 [arXiv:1311.1822] [INSPIRE]. 